Pontifícia Universidade Católica

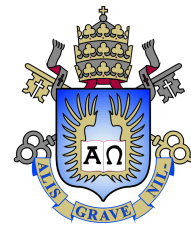

Maximiliano Enrique Contreras Lopez

\title{
Complete Synchronization in Delayed Coupled \\ Map Lattices
}

Dissertation presented to the Programa de Pós-graduação em Física of PUC-Rio in partial fulfillment of the requirements for the degree of Mestre em Ciências - Física.

Advisor: Profa. Celia Beatriz Anteneodo de Porto 


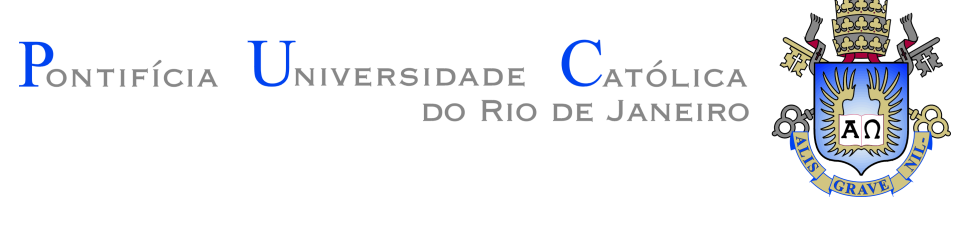

Maximiliano Enrique Contreras Lopez

\section{Complete Synchronization in Delayed Coupled \\ Map Lattices}

Dissertation presented to the Programa de Pós-graduação em Física of PUC-Rio in partial fulfillment of the requirements for the degree of Mestre em Ciências - Física. Approved by the undersigned Examination Committee.

Profa. Celia Beatriz Anteneodo de Porto

Advisor

Departamento de Física - PUC-Rio

Prof. Nuno Miguel Melo Crokidakis Peregrino

UFF

Profa. Sabrina Camargo

Universidade Federal do ABC

Prof. Márcio da Silveira Carvalho

Vice Dean of Graduate Studies Centro Técnico Científico PUC-Rio

Rio de Janeiro, November, 28th, 2017. 
All rights reserved.

\section{Maximiliano Enrique Contreras Lopez}

The author is graduated in Physics and Engineering from the Pontificia Universidad Católica de Chile, Santiago - Chile.

Bibliographic data

Contreras Lopez, Maximiliano Enrique

Complete Synchronization in Delayed Coupled Map Lattices / Maximiliano Enrique Contreras Lopez; advisor: Celia Beatriz Anteneodo de Porto. - Rio de janeiro: PUC-Rio , Departamento de Física, 2017.

v., 75 f: il. color. ; $30 \mathrm{~cm}$

Dissertação (mestrado) - Pontifícia Universidade Católica do Rio de Janeiro, Departamento de Física.

Inclui bibliografia

1. Physics - Teses. 2. Dynamical systems - Teses. 3. Complex systems - Teses. 4. Coupled map lattices - Teses. 5. Complete synchronization - Teses.

6. Sistemas complexos. 7. Redes de mapas acoplados. 8. Sincronização completa. 9. Acoplamento advectivo-difusivo. 10. Alcance das interações. I. Anteneodo de Porto, Celia Beatriz. II. Pontifícia Universidade Católica do Rio de Janeiro. Departamento de Física. III. Título. 


\section{Acknowledgments}

My deepest gratitude to my advisor Prof. Celia Anteneodo for her encouragement and trust. I am truly thankful for the opportunity she gave me to carry out this research.

I am in debt with the administrative staff of Departamento de $\mathrm{F}$ / sica at PUC-Rio and Prof. Hiroshi Nunokawa for their help and advice.

I want to thank my postgraduate colleagues for the companionship and their patience with the portunhol and my colleague Guillermo Gomez for his guidance and friendship.

Also I am gratefully appreciate the financial support given by $\mathrm{CNPq}$ and FAPERJ during this program. 


\section{Abstract}

Contreras Lopez, Maximiliano Enrique; Anteneodo de Porto, Celia Beatriz (Advisor). Complete Synchronization in Delayed Coupled Map Lattices. Rio de Janeiro, 2017. 75p. Dissertação de Mestrado - Departamento de Física, Pontifícia Universidade Católica do Rio de Janeiro.

We study complete synchronization in rings of logistic maps, using an advection-diffusion coupling scheme. The range of the interactions decays algebraically with distance between maps, sweeping from the totally connected to nearest-neighbor model, with synchronous or delayed lattice update. We studied the effects of delays and advection separately and combined. Although numerical studies were performed using the logistic map as local dynamics, some analytical results are more general. In the synchronous dynamics, synchronized states are chaotic, while the presence of delays allows synchronization in regular orbits. However, a strong contribution of delays in the updating can also produce chaotic synchronization. In all cases, longer interaction range favors synchronization, advection hinders synchronization and delays compensate the destructive effects of advection.

\section{Keywords}

Complex systems; Coupled map lattices; Complete synchronization; Advective diffusive coupling; Range of the interactions. 


\section{Resumo}

Contreras Lopez, Maximiliano Enrique; Anteneodo de Porto, Celia Beatriz. Sincronização completa em redes de mapas acoplados com retardo. Rio de Janeiro, 2017. 75p. Dissertação de Mestrado - Departamento de Física, Pontifícia Universidade Católica do Rio de Janeiro.

Estudamos sincronização completa em aneis de mapas logísticos, usando um esquema de acoplamento advectivo-difusivo. O alcance das interações diminui algebricamente com a distância entre mapas, varrendo do caso to-talmente conectado ao de primeiros vizinhos, com atualização sincronizada ou com retardo. Estudamos os efeitos do retardo e da adveç̧ão separada-mente e combinados. Embora os estudos numéricos tenham sido realizados utilizando o mapa logístico como dinâmica local, alguns resultados analí-ticos são mais gerais. Na dinâmica síncrona, os estados sincronizados são caóticos, enquanto a presença de atrasos permite a sincronização em órbi-tas regulares. No entanto, uma forte contribuição de retardos na atualização também pode produzir sincronização caótica. Em todos os casos, as inte-rações de longo alcance favorecem a sincronização, a advecção dificulta a sincronização, e os atrasos podem compensar os efeitos destrutivos da ad-vecção.

\section{Palavras-chave}

Sistemas complexos; Redes de mapas acoplados; Sincronização completa; Acoplamento advectivo-difusivo; Alcance das interações. 


\section{Table of contents}

1 Introduction 14

2 The Models $\quad 18$

2.1 Delayed Advective Diffusive Model (DAD) 18

2.2 Synchronous Advective Diffusive Model (SAD) 21

2.3 Delayed Purely Diffusive Model (DPD) 22

2.4 Synchronous Purely Diffusive Model (SPD) 22

3 Analytical results $\quad 23$

3.1 Synchronous Purely Diffusive (SPD) 23

3.2 Synchronous Advective Diffusive (SAD) 28

3.3 Delayed Purely Diffusive (DPD) 32

3.4 Delayed Advective Diffusive (DAD) 36

$4 \quad$ Numerical Results $\quad 39$

$\begin{array}{lll}4.1 & \text { Simulations setup } & 39\end{array}$

$\begin{array}{lll}4.2 & \text { Collective metrics } & 39\end{array}$

4.3 Results for the Synchronous Purely Diffusive model 40

4.4 Results for the Synchronous Advective Diffusive model 43

4.5 Results for the Delayed Purely Diffusive model 46

4.6 Results for the Delayed Advective Diffusive model 51

5 Conclusions and Perspectives $\quad 55$

$\begin{array}{ll}5.1 \text { Conclusions } & 55\end{array}$

$\begin{array}{lll}5.2 & \text { Perspectives } & 56\end{array}$

A Appendix A: Numerical results for the SPD model $\mathbf{5 9}$

B Appendix B: Numerical results for the SAD model $\quad 60$

C Appendix C: Numerical results for the DPD model 63

D Appendix D: Numerical results for the DAD model $\quad 66$

E Appendix E: Other numerical results $\quad 72$

$\begin{array}{ll}\text { Bibliography } & 73\end{array}$ 


\section{List of figures}

$\begin{array}{lll}\text { Figure 1.1 Illustrative uncoupled elements of a system. } & 15\end{array}$

$\begin{array}{lll}\text { Figure 1.2 Two types of phase synchronization. } & 15\end{array}$

1.2(a)In-phase synchronization $\quad 15$

1.2(b)Anti-phase synchronization $\quad 15$

$\begin{array}{lll}\text { Figure } 1.3 & \text { Completely synchronized state. } & 16\end{array}$

Figure 2.1 Small lattice example. The ring has periodic boundaries. 18

Figure 2.2 Equivalent totally connected ring when $\alpha=0 . \quad 20$

Figure 3.1 Heatmaps for the elements of $A_{i, j}$. 24

3.1(a)At $\alpha=0.1$, all $A_{i, j \neq i}>0.6 \quad 24$

3.1(b)As $\alpha=3, j=i \pm 1$ predominate $\quad 24$

Figure 3.2 The dependence of $A_{1, j}$ with $\alpha$. 25

Figure 3.3 Eigenvalues of $\Psi$ as function of $\alpha$. 26

Figure 3.4 Complete synchronization frontier. 28

Figure 3.5 Heatmaps for the elements of the matrix $B$. 29

$\begin{array}{ll}3.5(\text { a)Positive and negative regions } & 29\end{array}$

$3.5(\mathrm{~b}) B_{i, j}(3) \neq 0$ close to the diagonal $\quad 29$

Figure 3.6 Relation between $B_{1, j}$ and $\alpha . \quad 30$

Figure $3.7 \omega_{k}$ does not have a unique maximum for all $\alpha$. 31

Figure 3.8 CS theoretical frontiers for different values of $\gamma$. The CS

states, in each case, are located below the corresponding curve. 32

Figure 3.9 Theoretical CSR for the DPD, with $\beta=0.6$, and SPD

models. The red (continuous) line indicates the frontier for the

CSR of the synchronous model. The colored region below the curve corresponds to CS states for the SPD model. The green and blue lines (dotted) enclose the CSR for the delayed model, that includes short-range interactions, shown in the upper panel. 36

Figure 3.10 CSR for two illustrative cases of the delayed scheme, with and without advection.

3.10(a)CS states for $\beta=0.5$ and $\gamma=0.0$. 38

3.10 (b)CS states for $\beta=0.5$ and $\gamma=0.6$. 38

Figure 4.1 Collective metrics for $\alpha=0$ and $\alpha=3$. The red dots, representing $h_{t}$, correspond to 200 consecutive values after the transient $t_{0}$.

4.1(a)CS occurs for $\epsilon \geq 0.5$. The density of the orbits and the positive $\lambda$ indicate that the CS states are chaotic.

4.1(b)For range of interactions $\alpha \geq 1$, the synchronous schemes do not have CS states, regardless $\epsilon$.

Figure 4.2 CS states, frontier and $\sigma$ for the SPD model.

4.2(a)The red line corresponds to the theoretical frontier given by equation (3-17) and the dots to the CS states with $\sigma \leq 10^{-14}$ obtained by numerical simulations. 
4.2(b)The values of $\sigma$ in $(\alpha, \epsilon)$ show four different regions. The CS states are shown in white and the theoretical frontier in blue.

Figure 4.3 The Lyapunov exponent for the SPD model.

4.3(a)Simulations show two regions where $\lambda<0$, associated with regular orbits.

4.3(b)Values of $\lambda$ in the whole $(\alpha, \epsilon)$ space. As was predicted, the CS states have chaotic orbits, indicated by $\lambda>0$.

Figure 4.4 Collective metrics $h, \sigma$ and $\lambda$ for the SAD model.

4.4(a)For $\alpha=0$ and $\gamma=0.1$, CS occurs for $\epsilon \geq 0.51$. The density of the orbits and the positive $\lambda$ indicate that the CS states are chaotic.

4.4(b)For $\alpha=0$ and $\gamma=0.4$, CS occurs for $\epsilon \geq 0.57$.

Figure 4.5 CS states (dots) and theoretical frontier (red line) for

two $\gamma$ values of the SAD model show the reduction of the CSR.

Figure 4.6 Effect of advection. For each case, the same 12 individual trajectories, for 20 iterations after the transient time, are shown. 44 4.6(a)When $\gamma=0.4$ the spread out is observable and the CS state has been lost.

4.6(b)When $\gamma=0.8$ the spread becomes larger.

Figure 4.7 The heatmaps show average values in the parameter space. For low values of advection, they resemble those for SPD model.

4.7(a)Average value of $\sigma$ for SAD model with $\gamma=0.1$

4.7(b)Average value of $\lambda$ for SAD model with $\gamma=0.1$

Figure 4.8 Simulations show that increasing $\gamma$ reduce the number of states with $\lambda<0$.

$4.8(\mathrm{a}) \gamma=0.1$

$4.8(\mathrm{~b}) \gamma=0.3$

Figure 4.9 Effect of advection over a state with negative Lyapunov exponent. For each case, the same 12 individual trajectories, for 20 iterations after the transient time, are shown.

4.9(a)When $\gamma=0.1$ the trajectories start to spread out.

4.9(b)When $\gamma=0.3$ the state is not longer characterized by a NLE.

Figure 4.10 Collective metrics $h, \sigma$ and $\lambda$ at $\alpha=0$ for the DPD model. Simulations show that delay creates new types of orbits and change the CS states.

4.10(a) $\alpha=0, \beta=0.1$

4.10 (b) $\alpha=0, \beta=0.8$

Figure 4.11 Complete synchronization states for DPD model.

4.11(a)For $\beta=0.25$ the CSR is clearly larger than the CSR for the SPD model.

4.11(b)For $\beta=1$ the CSR is also larger that the one for the SPD model.

Figure 4.12 Negative Lyapunov exponent for the DPD model. 48

4.12(a)Small delay favors states with negative $\lambda$. 48

4.12(b)With large $\beta$ the number of NLE states decreases. 48 
Figure 4.13 For $\beta=0.4, \alpha=0$ the CS states, frontiers and collective metrics.

4.13(a) The dots correspond to the CS states. Inside the solid lines, orbits of period 1 are completely synchronized.

4.13(b)The value of $h$ show the type of orbit that are contained in the CSR in (a).

Figure 4.14 For $\beta=1$ the CS states and the collective metrics. In this case $\alpha=0$.

4.14(a)For $\beta$ large enough, the CSR is composed by two separated regions.

4.14(b) The value of $h$ show that the two CSR have contain regular orbits, but the one on the right can also have chaotic CS states.

Figure 4.15 Deviation and Lyapunov exponent heatmaps for the

DPD model when $\beta=0.4$.

4.15(a)Average values of $\sigma$ for $\beta=0.4 \quad 50$

4.15(b)Average values of $\lambda$ for $\beta=0.4 \quad 50$

Figure 4.16 For $\beta=1$, the overlap of regular and CS states. $\quad 50$

4.16(a)CS and NLE states together $\quad 50$

4.16(b) $\beta=1$ at $\alpha=0.5 \quad 50$

Figure 4.17 The Collective metrics $h, \sigma$ and $\lambda$ for two illustrative examples in the DAD model. 51

$4.17(\mathrm{a}) \alpha=0, \beta=0.1, \gamma=0.2 \quad 51$

$4.17(\mathrm{~b}) \alpha=0, \beta=0.8, \gamma=0.3 \quad 51$

Figure 4.18 The effect of advection when the delay parameter is fixed. 51

4.18(a)DPD case with $\beta=0.1$ and $\gamma=0 \quad 51$

4.18(b)DAD case with $\beta=0.1$ and $\gamma=0.4 \quad 51$

Figure 4.19 Heatmaps for the DAD model. $\quad 52$

4.19(a)Lyapunov exponent $\quad 52$

4.19(b)Deviation $\quad 52$

Figure 4.20 Short-range sensitivity to advection. $\quad 53$

4.20(a)DPD case with $\beta=0.7 \quad 53$

4.20(b)DAD case with $\beta=0.7$ and $\gamma=0.2 \quad 53$

Figure 4.21 The top plot shows the result without advection. The advection is increased downwards. This shows that the type of orbits are almost the same as the DPD case. The advection have a little $(\epsilon=0.4)$ or no effect $(\epsilon>0.6)$ in presence of delay. In this case, $\alpha=0$.

Figure 4.22 Effect of $\gamma$ on the collective metrics. In this case $\alpha=0 . \quad 54$

Figure 5.1 Isolated effect of advection in the SAD model. 56

5.1(a)Advection on CS states. $\quad 56$

5.1(b)Advection on NLE states. $\quad 56$

Figure 5.2 The effect of delay on CS states. 56

$\begin{array}{ll}5.2(\text { a)CSR states with } \beta=0.3 & 56\end{array}$

5.2(b)CSR shrinks for $\beta>0.5 \quad 56$

Figure 5.3 CPS appears in the CS zone. 58

5.3(a)Points where $\phi=1 \quad 58$

5.3(b)Heatmap for $\phi \quad 58$ 
Figure $5.4 \phi$ becomes homogeneous with increasing advection.

5.4(a)Heatmap of $\phi, \gamma=0.2 \quad 58$

$5.4(\mathrm{~b}) \phi=1, \gamma=0.5 \quad 58$

Figure A.1 The green zone of Figure 4.2(b) has non periodic clusters and quasi synchronized states.

A.1(a) $(\alpha, \epsilon)=(0.0,0.4) \quad 59$

A.1(b) $(\alpha, \epsilon)=(0.5,0.6) \quad 59$

Figure A.2 Yellow and white zones of Figure 4.2(b). 59

A.2(a) Yellow zone $(\alpha, \epsilon)=(1.5,0.8) \quad 59$

A.2(b) White zone $(\alpha, \epsilon)=(0.3,0.8)$

Figure B.1 Collective metrics $h, \sigma$ and $\lambda$ for the SAD model at $\alpha=0$. Advection causes the reduction of the synchronization domain. Each panel correspond to a different value of $\gamma$.

Figure B.2 In the left column the theoretical frontier and the complete synchronization region (CSR) from simulations. The effect of advection, from $\gamma=0.1$ to 0.7 is to reduce the CSR. In the right column the average value for $\sigma$ in the parameter space is shown.

Figure B.3 Lyapunov exponent values for the SAD model. In the left column, the states with negative Lyapunov exponent (NLE) which are modified by the effect of advection: advection destruct this type of states. In the right column, the average value for $\lambda$ in the parameter space is shown.

Figure C.1 Collective metrics $h, \sigma$ and $\lambda$ in the DPD model for $\alpha=0$. The delay favors synchronization. Large enough $\beta$ makes chaotic orbits emerge, as shown in the last panel in the right column.

Figure C.2 In the left column, CS states for different values of $\beta$ are shown. In all cases, the delay favors the synchronization. In the right column, the average value of $\sigma$ for different values of $\beta$ is shown in the parameter space.

Figure C.3 Lyapunov exponent values for the DPD model. In the left column, states with negative Lyapunov exponent are modified by the effect of delay. In the right column the average value for $\lambda$ is shown for different values of $\beta$, in the parameter space.

Figure D.1 Collective metrics $h, \sigma$ and $\lambda$ in the DAD model for $\alpha=0$. In the left column for $\beta=0.1, \gamma$ goes from 0.1 (upper panel) to 0.4 (lower panel). In the right column for $\beta=0.3, \gamma$ goes from 0.1 (upper panel) to 0.4 (lower panel). The advection reduces the CS domain.

Figure D.2 Collective metrics $h, \sigma$ and $\lambda$ in the DAD model for $\alpha=0$. In the left column for $\beta=0.7, \gamma$ goes from 0.1 (upper panel) to 0.4 (lower panel). In the right column for $\beta=0.9, \gamma$ goes from 0.1 (upper panel) to 0.4 (lower panel). The advection reduces the CS domain. 
Figure D.3 CS states in the DAD model. In the left column for $\beta=0.1, \gamma$ goes from 0.1 (upper panel) to 0.8 (lower panel). In the right column for $\beta=0.3, \gamma$ goes from 0.1 (upper panel) to 0.8 (lower panel). The advection reduces the CS domain while the delay favor CS states.

Figure D.4 CS states in the DAD model. In the left column for $\beta=0.7, \gamma$ goes from 0.1 (upper panel) to 0.8 (lower panel). In the right column for $\beta=0.9, \gamma$ goes from 0.1 (upper panel) to 0.8 (lower panel). The advection reduces the CS domain while the delay favor CS states.

Figure D.5 Negative Lyapunov exponent in the DAD model. In the left column for $\beta=0.1, \gamma$ goes from 0.1 (upper panel) to 0.8 (lower panel). In the right column for $\beta=0.3, \gamma$ goes from 0.1 (upper panel) to 0.8 (lower panel). The advection reduces the number of this type of states.

Figure D.6 Negative Lyapunov exponent in the DAD model. In the left column for $\beta=0.7, \gamma$ goes from 0.1 (upper panel) to 0.8 (lower panel). In the right column for $\beta=0.9, \gamma$ goes from 0.1 (upper panel) to 0.8 (lower panel). The advection reduces the number of this type of states.

Figure E.1 For $\beta=0.2$, in the DAD model, the effect of $\gamma$ in the collective metrics is shown. When $\alpha=50$, the advection only reduce the domain in $\epsilon$.

Figure E.2 The same phenomenon described above occurs for $\beta=$ 0.4. This means that the effect of the range of interactions is stronger than the effect of delay and advection. 


\section{List of Abreviations}

CML - Coupled Map Lattice

CS - Complete Synchronization

CSR - Complete Synchronization Region

CSF - Complete Synchronization Frontier

LE - Lyapunov Exponent

LS - Lyapunov Spectrum

NLE - Negative Lyapunov Exponent

SPD - Synchronous Purely Diffusive

SAD - Synchronous Advective Diffusive

DPD - Delayed Purely Diffusive

DAD - Delayed Advective Diffusive 


\section{1 \\ Introduction}

Chaos and synchronization are two words that seem to be in opposite ends of our reality. However, the combination of these two concepts is at the center of the emergence of patterns and structures in complex systems.

The earliest register of synchronization was made by Christiaan Huygens in 1665. He observed that two pendulum clocks with a common support progressively matched their oscillations until perfectly coincide [1]. Since then, the synchronization phenomenon has been observed in several fields from arrays of Josephson junctions to fireflies and brain oscillations [2-4]. We will understand synchronization as [1]: “..adjustment of rhythms of oscillating objects due to their weak interaction."

In quantitative terms, we should measure that adjustment to estimate the level of synchronization of any two objects. If this measurement coincides through time, we can say that the objects are synchronized.

In this work we will focus on the synchronization of a specific type of objects, chaotic maps. When two or more chaotic maps interact, either due to coupling or forcing, they can become in synchrony. This phenomenon has been largely studied and depending on the type of interaction between the systems, unidirectional or bidirectional, the type of synchronization can be classified as: complete, phase, lag, generalized, intermittent lag, imperfect phase and almost synchronization. Among those, we are interested in complete synchronization (CS). An extensive review of synchronization of chaotic systems and types of synchronizations can be found in [5].

In order to illustrate complete synchronization, let us imagine that we are measuring a certain feature $x$ of each element of a given dynamical system with a frequency of one time unit. So, for each time step, we plot the value of $x$ of each element of the system. 


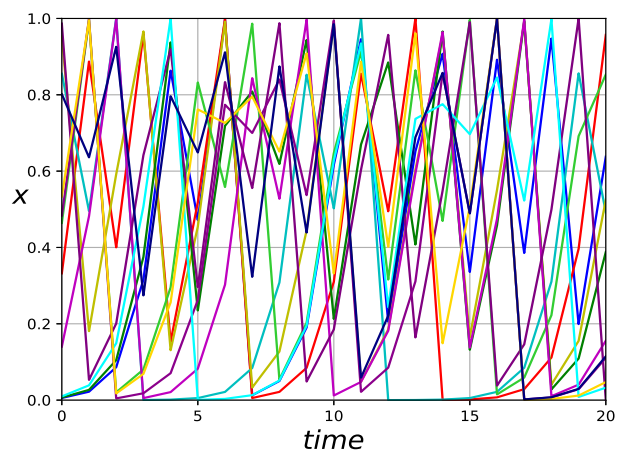

Figure 1.1: Illustrative uncoupled elements of a system.

If the coupling is too weak, each system will follow its own dynamics as in Figure 1.1. As we change parameters of the system, the collective behavior can change.

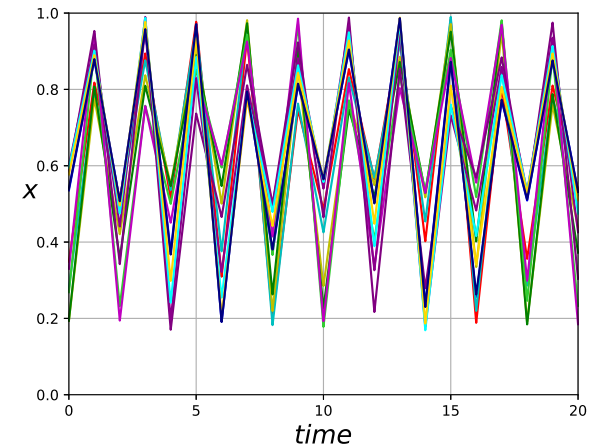

1.2(a): In-phase synchronization

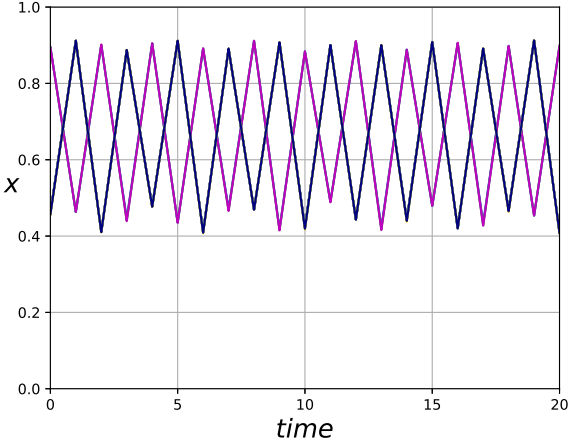

1.2(b): Anti-phase synchronization

Figure 1.2: Two types of phase synchronization.

Figure 1.2 shows two types of phase synchronization for the same system. In (a), the elements do not have the same value or state in each measurement, but they have the same orientation. On the contrary, in (b) the elements are in opposite phases. Both plots have as a common characteristic that they are periodic. Nevertheless, that is not a prerequisite for synchronization. Synchronization does not require periodic orbits. In fact we will see that chaotic synchronized orbits can also occur.

Figure 1.3 shows the same elements but completely synchronized. All the elements are doing exactly the same at the same time. This generates the superposition of individual trajectories. Mathematically, if we label the $N$ elements of the system, $i=1, \ldots, N$, the CS state is defined as $x^{1}(t)=x^{2}(t)=$ $\cdots=x^{N}(t),[6,7]$. 


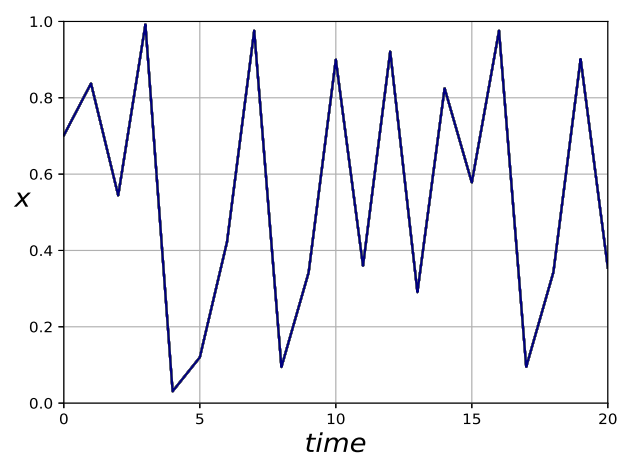

Figure 1.3: Completely synchronized state.

A standard model to study the synchronization of chaotic systems is a Coupled Map Lattice (CML) [8], a regular array whose elements are maps. A CML allows to study spatiotemporal patterns: periodic, quasi periodic, intermittent, chaotic, synchronized, chimeras, traveling and frozen patterns $[9,10]$. Then, the effects of the internal parameters of the local map, as well as of different types of interactions can be investigated.

The most extensively studied CML is the 1-dimensional lattice with periodic boundary conditions (that is, a ring). Although there are some studies that include intermediate ranges $[11,12]$, most studies focus on either nearestneighbors or global interactions [13-15]. Of special interest are interactions that decay with the distance $r$, between maps in the lattice nodes, as $1 / r^{\alpha}$, with $\alpha \geq 0$. This functional form allows to scan continuously the range of interactions between the extreme cases.

Regarding the type of coupling, traditionally the one considered is diffusive (symmetric), as will be defined in Chapter 2. The incorporation of advection (asymmetric coupling) in this type of model is not frequent. Nonetheless, in the series [16-19] the advective-diffusive model for nearest neighbors is obtained from the continous differential equation. For this model, pattern formation is studied. To our knowledge, the globally coupled or long-range interacting advective-diffusive CML has not been studied in the literature.

Another traditional feature in CML is the use of synchronous update schemes, in contrast with the introduction of time delays, to account for finite propagation times in information transmission [20, 21].

The present work is based on the methodology and the findings in [20, 21], extending them to the advective-diffusive model, with and without delay. Our aim is to incorporate all these features into one CML and explore the mixed effect. 
The remainder of this dissertation is organized as follows.

In Chapter 2 we introduce the mathematical models that describe the couplings, purely diffusive and advective-diffusive, and the update schemes, synchronous and delayed. In Chapter 3 we present the analytical results for each model. In Chapter 4 the results from the dynamical simulations for each model are presented and compared with the corresponding analytical results from Chapter 3. Finally, in Chapter 5, we summarize the obtained results and their implications. 


\section{2}

\section{The Models}

In this chapter we define all the models used in the present work. We start with the most general case, the Delayed Advective Diffusive (DAD) scheme, explaining the role of each variable and parameter. Then the other models are obtained as particular cases.

\section{1}

\section{Delayed Advective Diffusive Model (DAD)}

A Coupled Map Lattice, or CML, is a dynamical system with discrete time, discrete space and continuous state variables [8]. In simple terms, it is an arrange of nodes or elements in a, usually but not necessarily, regular configuration (discrete space). The temporal evolution of each element occurs in regular time steps (discrete time) and is given by a map (with continuous states). If these nodes are connected or coupled, then they can interact with each other.

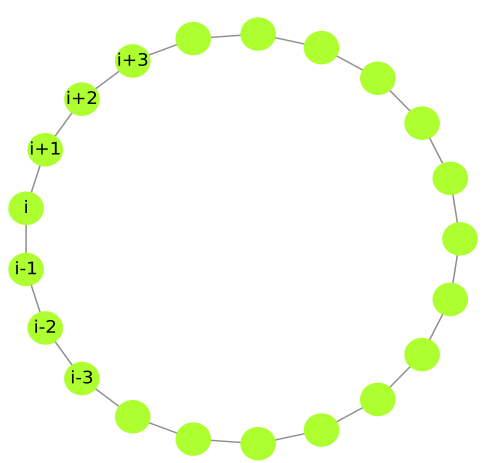

Figure 2.1: Small lattice example. The ring has periodic boundaries.

For a lattice of size $N$, we can label each node as in Figure 2.1. So at time $t \in \mathbb{Z}_{0}^{+}$, we can define the state of the $i$-th node as $x_{t}^{i}$. If the maps are uncoupled, each map is defined by a function $f$ that gives the rule to evolve from any time to the following

$$
x_{t+1}^{i}=f\left(x_{t}^{i}\right) .
$$


The next state of the system, at time $t+1$, is represented by the $N$-dimensional vector $x_{t+1}^{i}$. When the maps interact, coupling terms are added into equation $2-1$.

Let us call the global state of the lattice $\mathbf{x}_{t}=\left(x_{t}^{1}, x_{t}^{2}, \ldots, x_{t}^{N}\right)$. The previous states of the CML are given by the collection $\left\{\mathbf{x}_{t-1}, \ldots, \mathbf{x}_{0}\right\}$, where $\mathbf{x}_{\mathbf{0}}$ is an arbitrary initial random state and $\mathbf{x}_{t}$ is the current state of the system. The rule to find the next state of the system can depend on the current state, previous states and a set of parameters $\zeta$,

$$
\mathbf{x}_{t+1}=\mathcal{F}\left(\mathbf{x}_{t}, \mathbf{x}_{t-1}, \ldots, \mathbf{x}_{0}, \zeta\right) .
$$

If this relation can be written only using $\mathbf{x}_{t}$ and $\zeta$, as

$$
\mathbf{x}_{t+1}=\mathcal{F}\left(\mathbf{x}_{\mathbf{t}}, \zeta\right),
$$

we consider that the system has no delay or the update scheme is synchronous. When the equation includes not only $\mathbf{x}_{t}$ but previous states, as in (2-2) we will say that the update scheme has delay or is delayed. The inclusion of delay makes sense when the system is large because the information between distant nodes should take finite time to travel.

Furthermore, distant elements should have smaller mutual influence, compared with those located in the vicinity, called nearest neighbors. In order to model this last phenomenon, we define the distance and a range parameter. The distance between the $i$-th and $j$-th elements in the lattice, $r_{i, j}$, is defined as the number of edges in the shortest path between these two nodes. This can be written as

$$
r_{i, j}=\min _{k}|i-j+k N|,
$$

where $k$ is the natural number that minimizes equation (2-4). In the example of Figure 2.1, seven elements have been labeled from $i-3$ to $i+3$, where $i$ can be any element $(i=1 \ldots N)$. The distances are, for instance, $r_{i, i-2}=2$, $r_{i, i+1}=1, r_{i, i+3}=3$.

To control the range of the interactions, we introduce the range parameter $\alpha \in \mathbb{R}_{\mathbb{0}}^{+}$. The influence $K_{i, j}$ of the element $j$ over the element $i$ will be proportional to the inverse of the distance to power $\alpha$, i.e.,

$$
K_{i, j} \propto \frac{1}{r_{i, j}^{\alpha}} .
$$

Using the parameter $\alpha$, we can select the range from nearest neighbors when $\alpha \rightarrow \infty$, to global when $\alpha \rightarrow 0$. In this limit, all the elements influence each other equally regardless of the distance $r_{i, j}$ between them. This can be seen as a totally connected graph, where each node is directly connected with all the others, as represented in Figure 2.2. 


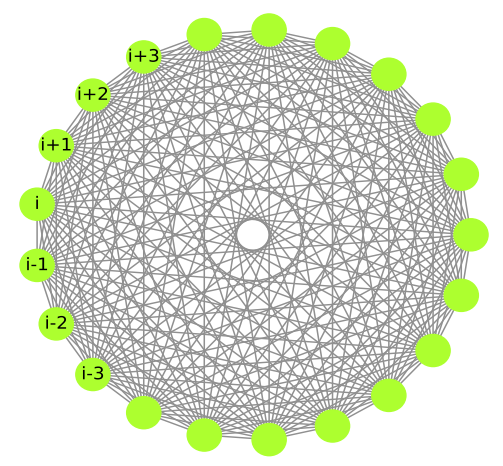

Figure 2.2: Equivalent totally connected ring when $\alpha=0$.

For the present study we introduce delay using an intermediate state variable $\hat{\mathbf{x}}$, defined as

$$
\hat{\mathbf{x}}_{t}=\beta \mathbf{x}_{t-1}+(1-\beta) \mathbf{x}_{t} .
$$

The delay parameter $\beta \in[0,1]$, controls the contribution of a onetime delay, going from synchronous update when $\beta=0$, to a purely delayed dynamics when $\beta=1[20,21]$.

As local dynamics, we use the logistic map $f(x)=4 x(1-x)$, with $x \in[0,1]$. This is a well known and extensively studied map that exhibits chaotic behavior [22-24]. $N$ of these elements are arranged in a ring (onedimensional lattice with periodic boundary conditions) as depicted in the example of Figure 2.1.

We consider two types of coupling schemes: diffusive and advective. We define the diffusive scheme as the combined (symmetric) effect of those nodes $i+j$ and $i-j$ over $i$ as

$$
S_{i, j} \propto \frac{f\left(x^{i+j}\right)+f\left(x^{i-j}\right)}{r_{i, j}^{\alpha}} .
$$

The advective (asymmetric) scheme is given by the differential effect of the same nodes as

$$
V_{i, j} \propto \frac{f\left(x^{i+j}\right)-f\left(x^{i-j}\right)}{r_{i, j}^{\alpha}} .
$$

To control the strength of couplings, we introduce two parameters: the coupling or diffusive parameter $\epsilon$ and the advective parameter $\gamma$. These two parameters can be connected with physical quantities through the discretization of the one-dimensional continuous advective-diffusive equation, as shown in $[19]$. 
Now, considering all the elements of the lattice, we can state the Delayed Advective Diffusive model adding up the influence of diffusive and advective terms of all the $N-1$ elements over the node $i$, together with its local dynamics,

$$
x_{t+1}^{i}=(1-\epsilon) f\left(x_{t}^{i}\right)+\frac{\epsilon}{\eta} \sum_{j=1}^{M} \frac{f\left(\hat{x}_{t}^{i+j}\right)+f\left(\hat{x}_{t}^{i-j}\right)}{r_{i, j}^{\alpha}}+\frac{\gamma}{\eta} \sum_{j=1}^{M} \frac{f\left(\hat{x}_{t}^{i+j}\right)-f\left(\hat{x}_{t}^{i-j}\right)}{r_{i, j}^{\alpha}} .
$$

The first term in equation (2-9) represents the contribution of the local dynamics. If we set $\epsilon$ and $\gamma$ both zero, the system would consist in a set of $N$ uncoupled chaotic logistic maps. The second term is the diffusive term. The relative strength of this term is controlled by the parameter $\epsilon$. The third term corresponds to the advective term. In this case, the strength is controlled by the parameter $\gamma$ whose values are restricted to the range $\gamma \in[-\epsilon, \epsilon]$, as shown in [19]. This restriction connects the advective parameter with the diffusive one.

There are two more elements that need to be defined in equation (2-9): $\eta$ and $M$. The parameter $\eta$ is a normalization factor defined as

$$
\eta(\alpha)=2 \sum_{j=1}^{M} \frac{1}{r_{i, j}^{\alpha}}
$$

Using the fact that the geometry for the model is symmetric (a ring), the sum over all the elements can be done defining $M=N / 2$, when $N$ is even and $M=(N-1) / 2$ when $N$ is odd.

Now, we will look at some particular cases of the model in (2-9).

\section{2}

\section{Synchronous Advective Diffusive Model (SAD)}

If we set $\beta=0$ in equation (2-9) we get that $\hat{x}_{t}=x_{t}$, and therefore

$$
x_{t+1}^{i}=(1-\epsilon) f\left(x_{t}^{i}\right)+\frac{\epsilon}{\eta} \sum_{j=1}^{M} \frac{f\left(x_{t}^{i+j}\right)+f\left(x_{t}^{i-j}\right)}{r_{i, j}^{\alpha}}+\frac{\gamma}{\eta} \sum_{j=1}^{M} \frac{f\left(x_{t}^{i+j}\right)-f\left(x_{t}^{i-j}\right)}{r_{i, j}^{\alpha}} .
$$

In this model the next state of the system, $\mathbf{x}_{t+1}$, depends only on the current state of the system $\mathbf{x}_{t}$, and the set of parameters. The update scheme is synchronous. The interaction remains advective $(\gamma)$ and diffusive $(\epsilon)$. 


\section{3}

\section{Delayed Purely Diffusive Model (DPD)}

Starting with equation (2-9), if we set $\gamma=0$,

$$
x_{t+1}^{i}=(1-\epsilon) f\left(x_{t}^{i}\right)+\frac{\epsilon}{\eta} \sum_{j=1}^{M} \frac{f\left(\hat{x}_{t}^{i+j}\right)+f\left(\hat{x}_{t}^{i-j}\right)}{r_{i, j}^{\alpha}} .
$$

In this case, we retain the delay through $\hat{\mathbf{x}}_{t}$, defined in equation 2-6, but exclude the advective term. The interaction is only diffusive.

\section{4}

\section{Synchronous Purely Diffusive Model (SPD)}

The simplest model is obtained from equation (2-9) setting both, $\beta$ and $\gamma$ equal to zero simultaneously.

$$
x_{t+1}^{i}=(1-\epsilon) f\left(x_{t}^{i}\right)+\frac{\epsilon}{\eta} \sum_{j=1}^{M} \frac{f\left(x_{t}^{i+j}\right)+f\left(x_{t}^{i-j}\right)}{r_{i, j}^{\alpha}} .
$$

It does not contain delay nor advection. 


\section{3}

\section{Analytical results}

In this chapter we analyze the emergence and stability of completely synchronized states (CS) in CMLs. Complete synchronization occurs when all the elements of the lattice adopt the same value, let us call it $x_{t}^{\star}$, for all times $t$, after a transient time $t_{0}$. That is $x_{t}^{1}=x_{t}^{2}=\ldots=x_{t}^{N}=x_{t}^{\star}$, for all $t>t_{0}$.

In order to determine the (linear) stability of these states, we analyze the tangent dynamics, that is the dynamics of small deviations.

We begin with the simplest CML, using the Synchronous Purely Diffusive (SPD) scheme, and from there we build results up to the Delayed Advective Diffusive (DAD) scheme. This is intended to give a comprehensive and incremental construction of the results.

\section{1}

\section{Synchronous Purely Diffusive (SPD)}

Governed by the SPD scheme, the coupled maps evolve according to

$$
x_{t+1}^{i}=(1-\epsilon) f\left(x_{t}^{i}\right)+\frac{\epsilon}{\eta} \sum_{j=1}^{M} \frac{f\left(x_{t}^{i+j}\right)+f\left(x_{t}^{i-j}\right)}{r_{i, j}^{\alpha}} .
$$

If the system were in a CS state, where all the maps are in the state $x_{t}^{\star}$, then the system of equations (3-1) reduces to the single equation

$$
x_{t+1}^{\star}=f\left(x_{t}^{\star}\right),
$$

which is the dynamics of the uncoupled map. In particular, the lattice can synchronize following a chaotic trajectory.

The small deviations around this chaotic CS state, neglecting terms greater than $\mathcal{O}(1)$, are given by

$$
\delta x_{t+1}^{i}=\left[(1-\epsilon) \delta^{i j}+\frac{\epsilon}{\eta} A_{i j}\right] f^{\prime}\left(x_{t}^{\star}\right) \delta x_{t}^{j},
$$

where $A_{i j}$ are the elements of matrix $A$ given by $A_{i j}=\left(1-\delta_{i j}\right) / r_{i j}^{\alpha}$. The evolution of displacements can be written in compact form as

$$
\delta \mathbf{x}_{t+1}=\mathbf{F}_{t}^{D} \delta \mathbf{x}_{t},
$$

where

$$
\delta \mathbf{x}_{t}=\left(\delta x_{t}^{1}, \delta x_{t}^{2}, \ldots, \delta x_{t}^{N}\right)^{T}
$$


and

$$
\mathbf{F}_{t}^{D}=\left[(1-\epsilon) \mathbb{1}+\frac{\epsilon}{\eta} A\right] f^{\prime}\left(x_{t}^{\star}\right)
$$

Equation (3-5) shows that $\mathbf{F}_{t}^{D}$ depends on time $t$ through $f^{\prime}\left(x_{t}^{\star}\right)$ only and also, that tangent dynamics is ruled by the eigenvalues of the matrix $F_{t}^{D}$ which are directly related to those of $A$.

The structure of $A$ represents the diffusive coupling in a ring type lattice of size $N$.

$$
A(\alpha)=\left[\begin{array}{ccccc}
0 & \frac{1}{r_{1,2}^{\alpha}} & \frac{1}{r_{1,3}^{\alpha}} & \cdots & \frac{1}{r_{1, N}^{\alpha}} \\
\frac{1}{r_{2,1}^{\alpha}} & 0 & \frac{1}{r_{2,3}^{\alpha}} & \cdots & \frac{1}{r_{2, N}^{\alpha}} \\
\frac{1}{r_{3,1}^{\alpha}} & \frac{1}{r_{3,2}^{\alpha}} & 0 & \ldots & \frac{1}{r_{3, N}^{\alpha}} \\
\vdots & \vdots & \vdots & \vdots & \vdots \\
\frac{1}{r_{N, 1}^{\alpha}} & \frac{1}{r_{N, 2}^{\alpha}} & \frac{1}{r_{N, 3}^{\alpha}} & \cdots & 0
\end{array}\right]
$$

recalling that the distances were defined in equation (2-4) through $r_{i, j}=$ $\min _{k}|i-j+k N|$.

Three features stand out: this matrix is circulant, it is symmetric and, for fixed size of the lattice, the elements $A_{i, j}$ depend on the range parameter $\alpha$ only. We explore this last point changing the value of $\alpha$ and visualizing the elements of $A$ as a heatmap for a ring of size $N=201$.

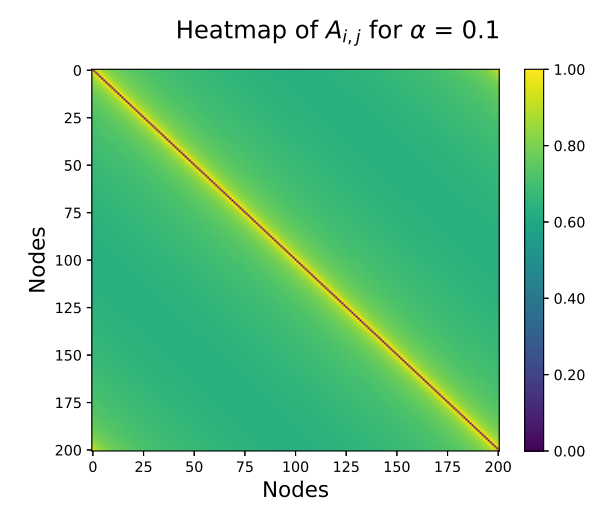

3.1(a): At $\alpha=0.1$, all $A_{i, j \neq i}>0.6$

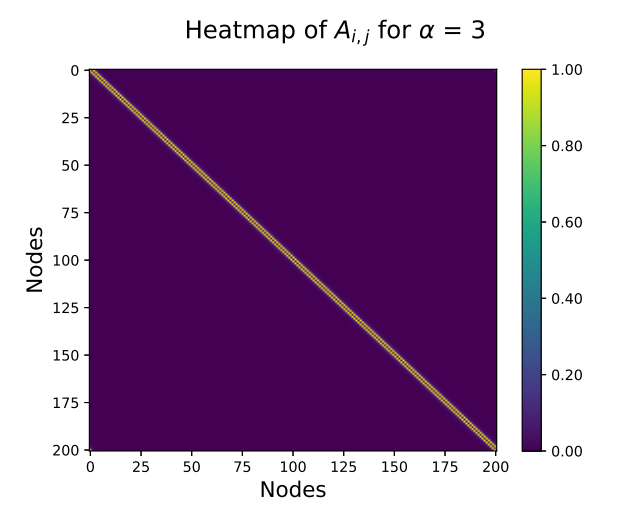

3.1(b): As $\alpha=3, j=i \pm 1$ predominate

Figure 3.1: Heatmaps for the elements of $A_{i, j}$.

The comparison between Figure 3.1(a) and 3.1(b) shows that the elements of $A$ decrease with $\alpha$. In (b), when $\alpha=3$, almost all the elements outside the diagonal are zero. To see in detail how the elements of $A$ change with $\alpha$, we use the fact that $A$ is a circulant matrix, so any row is a one-element shift of the preceding row. Then, we can analyze just one row. 
In Figure 3.2 all the elements of one row of the matrix $\mathrm{A}, A_{1, j}$, are plotted for different values of the parameter $\alpha$. Increasing $\alpha$ moves the curves down towards zero, with the exception of the borders $j=1, N$. Conversely, the contribution of all elements grows as $\alpha$ decreases. In the limit $\alpha=0$, all the elements of $A$ outside the diagonal are equal to 1 . This is represented by the red dotted curve.

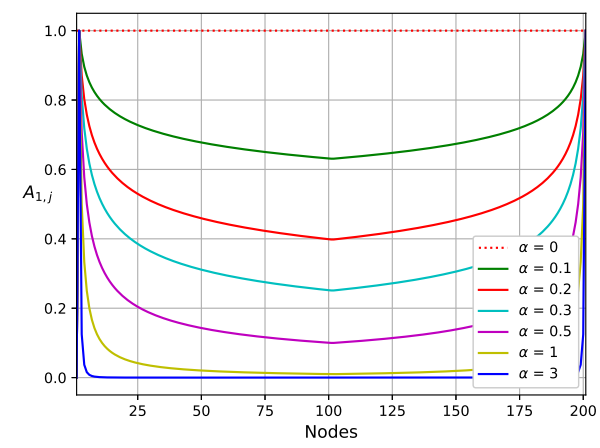

Figure 3.2: The dependence of $A_{1, j}$ with $\alpha$.

Since $A$ is circulant, it can be diagonalized using the Fourier matrix $U$, as $A / \eta=U \Psi U^{*}[6]$. The elements of the diagonal matrix $\Psi$ are

$$
\psi_{k}=\sum_{m=0}^{N-1} a_{m} e^{\frac{-2 \pi i m k}{N}}
$$

where $a_{m}$ is related with the elements of $A$ as

$$
a_{m}=\frac{A_{1, m+1}}{\eta} .
$$

and $\eta$ is the normalization factor defined in the previous chapter. The index $k$ enumerates the elements of the diagonal matrix $\Psi$.

In the case of odd $N, M=(N-1) / 2$ and the element $a_{m}$ is

$$
a_{m}= \begin{cases}0 & m=0 \\ \frac{1}{\eta m^{\alpha}} & m \in\left\{1, \ldots, \frac{N-1}{2}\right\} \\ \frac{1}{\eta(N-m)^{\alpha}} & m \in\left\{\frac{N+1}{2}, \ldots, N-1\right\} .\end{cases}
$$

Defining $W_{k} \equiv e^{\frac{-2 \pi i k}{N}}$, we can write $(3-7)$ as

$$
\psi_{k}=\sum_{m=1}^{M} \frac{1}{\eta m^{\alpha}} W_{k}^{m}+\sum_{m=M+1}^{N-1} \frac{1}{\eta(N-m)^{\alpha}} W_{k}^{m} .
$$

Through the change of variables $m=N-m^{\prime}$ in the second term of (3-9), we get

$$
\sum_{m=M+1}^{N-1} \frac{1}{\eta(N-m)^{\alpha}} W_{k}^{m}=\sum_{m^{\prime}=1}^{M} \frac{1}{\eta m^{\prime \alpha}} W_{k}^{N-m^{\prime}} .
$$


From this last expression, we notice that $W_{k}^{N}=1$. Putting it back in (3-9), we have

$$
\psi_{k}=\frac{1}{\eta} \sum_{m=1}^{M} \frac{W_{k}^{m}+W_{k}^{-m}}{m^{\alpha}}=\frac{2}{\eta} \sum_{m=1}^{M} \frac{\cos (2 \pi k m / N)}{m^{\alpha}} .
$$

This is the expression for the eigenvalues of $\Psi$ when $N$ is odd. It is clear that $\left|\psi_{k}\right| \leq 1$, for all $k \in 0, \ldots, N-1$.

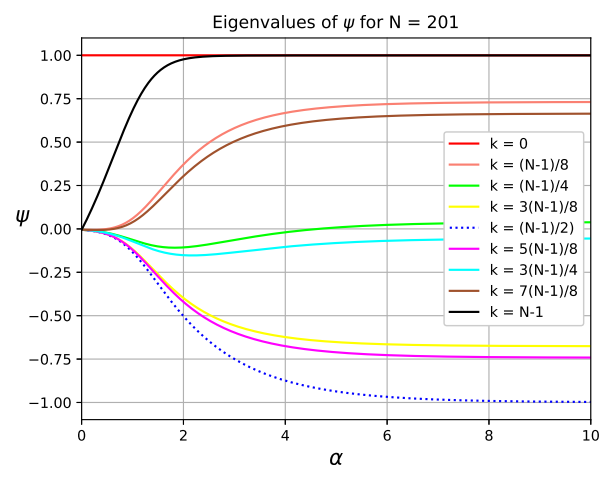

Figure 3.3: Eigenvalues of $\Psi$ as function of $\alpha$.

Illustrative values of $\psi_{k}$ for $\alpha \in[0,10]$ and $k=0, \ldots, N-1$, are shown in Figure 3.3. The red line and the dotted blue line correspond to the maximal and the minimal values for $\psi$, respectively. This is consistent with the results in Figure 3.2 where $k=0$ and $k=N-1$ are the maximal and second maximal values, while $k=(N-1) / 2$ corresponds to the minimum.

In the case of even $N$, the elements are

$$
a_{m}= \begin{cases}0 & m=0 \\ \frac{1}{\eta m^{\alpha}} & m \in\left\{1, \ldots, \frac{N}{2}-1\right\} \\ \frac{2}{\eta m^{\alpha}} & m=\frac{N}{2} \\ \frac{1}{\eta(N-m)^{\alpha}} & m \in\left\{\frac{N}{2}+1, \ldots, N-1\right\} .\end{cases}
$$

Using the same procedure, we can write the eigenvalues for this case as

$$
\psi_{k}=\frac{2}{\eta} \sum_{m=1}^{M} \frac{\cos (2 \pi k m / N)}{m^{\alpha}}-\frac{(-1)^{k}}{\eta(N / 2)^{\alpha}} .
$$

Equations 3-11 and 3-12 are general results for the diffusive interaction in a ring type lattice. The last term in equation 3-12 becomes negligible when $N$ is large enough.

To calculate the Lyapunov exponents of N-dimensional systems, with $N \geq 2$, we use the usual definition [25]

$$
\lambda=\lim _{n \rightarrow \infty} \frac{1}{n} \ln \left(\frac{\mathbf{y}_{n}}{\mathbf{y}_{0}}\right),
$$


where $\mathbf{y} \in \mathbb{R}^{N}$ is the displacement from the original orbit.

In CS states, the Lyapunov spectrum (LS) can be extracted from the evolution of initial tangent vectors. The product of Jacobian matrices at successive points in the trajectory [26],

$$
\delta \mathbf{x}_{t+1}=\mathbf{F}_{t}^{D} \delta \mathbf{x}_{t}=\mathbf{F}_{t}^{D} \mathbf{F}_{t-1}^{D} \ldots \mathbf{F}_{1}^{D} \mathbf{F}_{0}^{D} \delta \mathbf{x}_{0} .
$$

Using this last equation, together with equation (3-5), and the eigenvalues of the matrix $A$, we can get the Lyapunov spectrum for completely synchronized states, namely,

$$
\lambda_{k}^{*}=\lambda_{U}+\ln \left|(1-\epsilon)+\epsilon \psi_{k}\right|,
$$

with $k=0,1, \ldots, N-1$, and where $\lambda_{0}=\lambda_{U}$ is the Lyapunov exponent of the local map. The largest eigenvalue $\lambda_{U}$ is associated to the eigenvector $(1,1, \ldots, 1)$ which corresponds to the completely synchronized manifold, for any $\alpha$. Meanwhile, the transverse stability comes from the negativity of the remaining $(N-1)$ exponents [6]. This means that for $k \neq 0$,

$$
\left|(1-\epsilon)+\epsilon \psi_{k}\right|<e^{-\lambda_{U}} .
$$

From Figure 3.3, we use the fact that the curve $\psi$ is maximal $\left(\psi_{\max }\right)$, when $k=N-1$ (out of the case $k=0$, that gives the longitudinal stability), and minimal $\left(\psi_{\min }\right)$, when $k=(N-1) / 2$. Moreover, for the logistic map, we have $\lambda_{U}=\ln 2$. As the inequality must hold in all cases in between, then for $\epsilon$ we obtain

$$
\epsilon_{\min }=\frac{1-1 / 2}{1-\psi_{\max }} \leq \epsilon \leq \frac{1+1 / 2}{1-\psi_{\min }}=\epsilon_{\max } .
$$

This is the range for which complete synchronization is stable.

For instance, when $\alpha=0, \psi_{\max }=\psi_{\min }=0$, therefore $1 / 2 \leq \epsilon \leq 3 / 2$, that is $1 / 2 \leq \epsilon \leq 1$. When $\alpha \rightarrow \infty, \psi_{\max }=1$ and $\psi_{\min }=-1$, hence there is not allowed range of $\epsilon$.

Figure 3.4 shows the complete synchronization frontier. Below the curve there is a complete synchronization region (CSR), where CS states are chaotic. Notice that sufficiently long-range is required $(\alpha<0.75$, for $\epsilon \leq 1)$. 


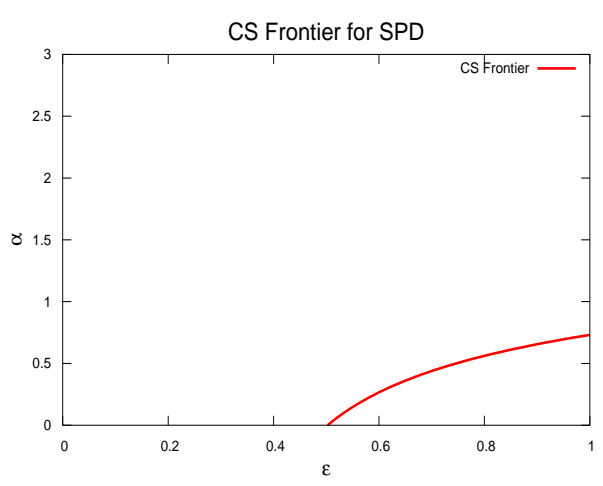

Figure 3.4: Complete synchronization frontier.

\section{2}

\section{Synchronous Advective Diffusive (SAD)}

The SAD scheme has an additional term, with respect to the SPD case, namely the advective (asymmetric) one, such that

$$
x_{t+1}^{i}=(1-\epsilon) f\left(x_{t}^{i}\right)+\frac{\epsilon}{\eta} \sum_{j=1}^{M} \frac{f\left(x_{t}^{i+j}\right)+f\left(x_{t}^{i-j}\right)}{r_{i, j}^{\alpha}}+\frac{\gamma}{\eta} \sum_{j=1}^{M} \frac{f\left(x_{t}^{i+j}\right)-f\left(x_{t}^{i-j}\right)}{r_{i, j}^{\alpha}} .
$$

Also in this case, in CS states equation (3-2) holds, therefore, it is worth to analyze the stability of chaotic CS states.

Again, the small deviations around a CS state, neglecting terms greater than $\mathcal{O}(1)$, are given by

$$
\delta x_{t+1}^{i}=\left[(1-\epsilon) \delta^{i j}+\frac{\epsilon}{\eta} A_{i j}+\frac{\gamma}{\eta} B_{i j}\right] f^{\prime}\left(x_{t}^{\star}\right) \delta x_{t}^{j},
$$

where $B_{i j}$ are elements of the matrix $B$. The elements of the first row of $B$ are given by

$$
B_{1 j}= \begin{cases}\left(1-\delta_{1 j}\right) / r_{1 j}^{\alpha} & j \leq(N-1) / 2 \\ -\left(1-\delta_{1 j}\right) / r_{1 j}^{\alpha} & j>(N-1) / 2\end{cases}
$$

The remaining rows can be obtained by shifting this first one because the matrix $B$ is circulant. The evolution of displacements can be written in compact form as

$$
\delta \mathbf{x}_{t+1}=\mathbf{F}_{t}^{A} \delta \mathbf{x}_{t}
$$

where

$$
\delta \mathbf{x}_{t}=\left(\delta x_{t}^{1}, \delta x_{t}^{2}, \ldots, \delta x_{t}^{N}\right)^{T}
$$

and 


$$
\mathbf{F}_{t}^{A}=\left[(1-\epsilon) \mathbb{1}+\frac{\epsilon}{\eta} A+\frac{\gamma}{\eta} B\right] f^{\prime}\left(x_{t}^{\star}\right)
$$

Equation (3-22) shows that $\mathbf{F}_{t}^{A}$ depends on time $t$ through $f^{\prime}\left(x_{t}^{\star}\right)$ and also, that the tangent dynamics is ruled by the eigenvalues of the matrix $\mathbf{F}_{t}^{A}$ which are directly related to those of $A$ and $B$. We already analyzed those of $A$, so we focus on the eigenvalues of matrix $B$, that represents the advective coupling in a ring type lattice, and is given by

$$
B(\alpha)=\left[\begin{array}{ccccc}
0 & \frac{1}{r_{1,2}^{\alpha}} & \ldots & \frac{-1}{r_{1, N-1}^{\alpha}} & \frac{-1}{r_{1, N}^{\alpha}} \\
\frac{-1}{r_{2,1}^{\alpha}} & 0 & \frac{1}{r_{2,3}^{\alpha}} & \ldots & \frac{-1}{r_{2, N}^{\alpha}} \\
\frac{-1}{r_{3,2}^{\alpha}} & \frac{-1}{r_{3,2}^{\alpha}} & 0 & \ldots & \frac{-1}{r_{3, N}^{\alpha}} \\
\vdots & \vdots & \vdots & \vdots & \vdots \\
\frac{1}{r_{N, 1}^{\alpha}} & \frac{1}{r_{N, 2}^{\alpha}} & \frac{1}{r_{N, 3}^{\alpha}} & \cdots & 0
\end{array}\right]
$$

There are important differences between the matrices $A$ and $B$. First, elements of $B_{i, j} \in[-1,1]$ while $A_{i, j} \in[0,1]$. Second, the matrix $A$ is symmetric, $A^{T}=A$, and $B$ is antisymmetric, $B^{T}=-B$. They have in common the fact that $\alpha$ plays the same role: increasing $\alpha$ reduces the absolute value of the elements outside the three main diagonals. This can be seen in the pictorial representation done in Figure 3.5.

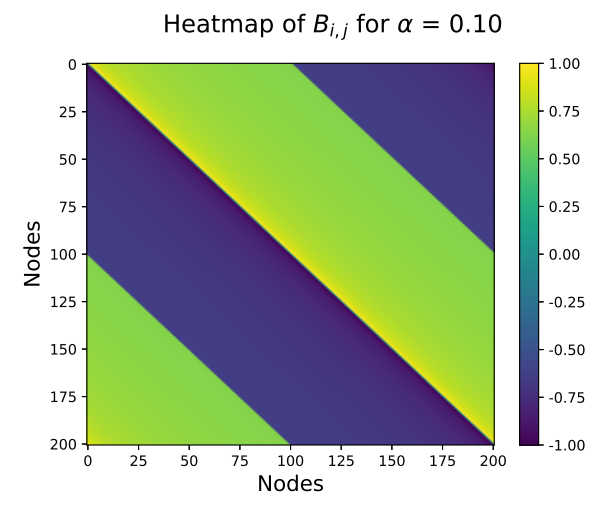

3.5(a): Positive and negative regions

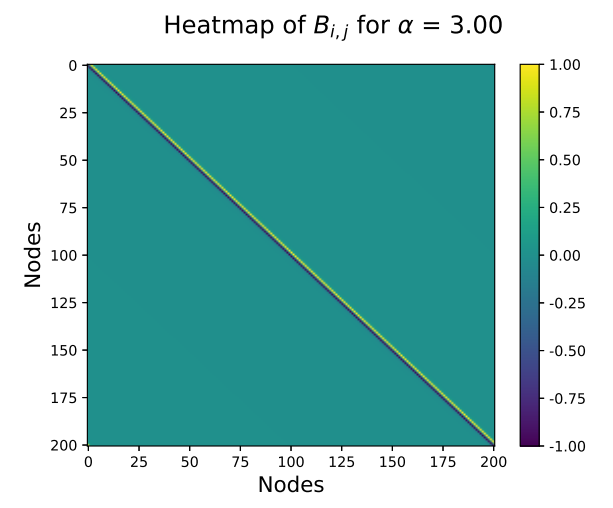

3.5(b): $B_{i, j}(3) \neq 0$ close to the diagonal

Figure 3.5: Heatmaps for the elements of the matrix $B$.

The matrix $B$ is also circulant, similarly to $\mathrm{A}$, and can be diagonalized in the same Fourier basis, as $B / \eta=U \Omega U^{*}$, where the diagonal elements of $\Omega$ are given by

with

$$
-i \omega_{k}=\sum_{m=0}^{N-1} b_{m} e^{\frac{-2 \pi i m k}{N}}
$$




$$
b_{m}=\frac{B_{1, m+1}}{\eta} .
$$

and the index $k$ enumerates the elements of the diagonal matrix $\Omega$.

When $N$ is odd, then $M=(N-1) / 2$ and,

$$
b_{m}= \begin{cases}0 & m=0 \\ \frac{1}{\eta m^{\alpha}} & m \in\left\{1, \ldots, \frac{N-1}{2}\right\} \\ \frac{-1}{\eta(N-m)^{\alpha}} & m \in\left\{\frac{N+1}{2}, \ldots, N-1\right\} .\end{cases}
$$

Replacing $b_{m}$ in $(3-23)$,

$$
\sum_{m=1}^{M} \frac{1}{\eta m^{\alpha}} W_{k}^{m}+\sum_{m=M+1}^{N-1} \frac{-1}{\eta(N-m)^{\alpha}} W_{k}^{m}
$$

Following the same procedure applied to $A$, we get

where

$$
\frac{1}{\eta} \sum_{m=1}^{M} \frac{W_{k}^{m}-W_{k}^{-m}}{m^{\alpha}}=\frac{-2 i}{\eta} \sum_{m=1}^{M} \frac{\sin (2 \pi k m / N)}{m^{\alpha}}=-i \omega_{k},
$$

$$
\omega_{k}=\frac{2}{\eta} \sum_{m=1}^{M} \frac{\sin (2 \pi k m / N)}{m^{\alpha}} .
$$

When $N$ is even, the elements of $B$ are

$$
b_{m}= \begin{cases}0 & m=0 \\ \frac{1}{\eta m^{\alpha}} & m \in\left\{1, \ldots, \frac{N}{2}-1\right\} \\ 0 & m=\frac{N}{2} \\ \frac{-1}{\eta(N-m)^{\alpha}} & m \in\left\{\frac{N}{2}+1, \ldots, N-1\right\} .\end{cases}
$$

The only difference between the two cases is that for $N$ even, when $m=N / 2, b_{m}=0$. Then, the eigenvalues are also $-i \omega_{k}$.

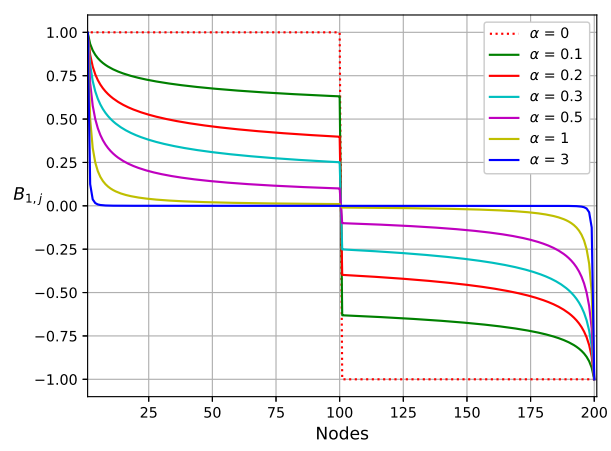

Figure 3.6: Relation between $B_{1, j}$ and $\alpha$.

Figure 3.6 shows that the extreme values for $\omega_{k}$ occur for different values of $k$ depending on $\alpha$. 
Again, in CS states, the Lyapunov spectrum (LS) can be extracted from the evolution of initial tangent vectors given by the product of Jacobian matrices at successive points in the trajectory,

$$
\delta \mathbf{x}_{t+1}=\mathbf{F}_{t}^{A} \delta \mathbf{x}_{t}=\mathbf{F}_{t}^{A} \mathbf{F}_{t-1}^{A} \ldots \mathbf{F}_{1}^{A} \mathbf{F}_{0}^{A} \delta \mathbf{x}_{0} .
$$

Using this last equation, together with equation (3-22), and the eigenvalues of the matrices $A$ and $B$, we can get the Lyapunov spectrum for completely synchronized states, namely,

$$
\lambda_{k}^{*}=\lambda_{U}+\ln \left|(1-\epsilon)+\epsilon \psi_{k}-i \gamma \omega_{k}\right|,
$$

with $k=0, \ldots, N-1$, and where $\lambda_{U}$ is the Lyapunov exponent of the local map. The eigenvalues $\omega_{k}$ correspond to the advective coupling. In Figure 3.7, we can see that maximal and minimal values of $\omega$ depend on $k$ and $\alpha$. This means that we can not use a single $\omega_{k}$ as the maximal or the minimal value.

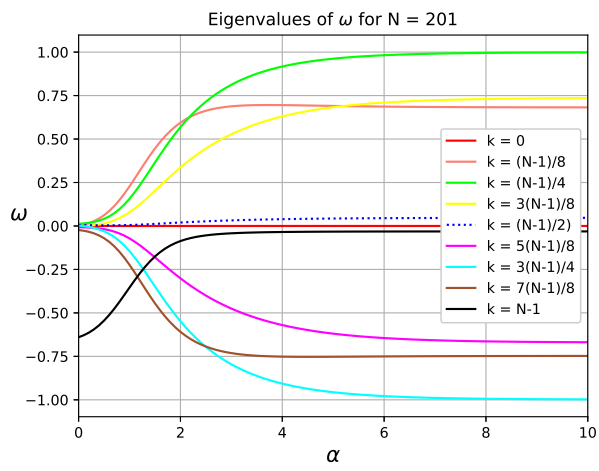

Figure 3.7: $\omega_{k}$ does not have a unique maximum for all $\alpha$.

When $k=0$, we have $\psi_{k}=1$ and $\omega_{k}=0$, therefore $\lambda_{k}^{*}=\lambda_{U}$. The eigenvalues for $k=0$ are associated to the eigenvector $(1,1, \ldots, 1)$ which corresponds to the completely synchronized manifold, for any $\alpha$. Meanwhile, the transverse stability comes from the negativity of the remaining $(N-1)$ exponents. This means that for $k \neq 0$,

$$
\left|(1-\epsilon)+\epsilon \psi_{k}-i \gamma \omega_{k}\right|<e^{-\lambda_{U}}
$$

that for the logistic map $\left(\lambda_{U}=\ln 2\right)$ becomes,

$$
\left[(1-\epsilon)+\epsilon \psi_{k}\right]^{2}+\left[\gamma \omega_{k}\right]^{2} \leq \frac{1}{4}
$$

For $\gamma=0$, we recover the inequality of the SPD model, as expected.

For given $\gamma$, we look for couples $(\alpha, \epsilon)$ such that $\psi_{k}=\psi(k, \alpha)$ and $\omega_{k}=\omega(k, \alpha)$ allow to satisfy simultaneously the inequality (3-31) and the restriction $-\epsilon \leq \gamma \leq \epsilon$, for all $k \in 0, \ldots, N-1$. If a couple verifies 
these conditions, we mark it as a complete synchronization point (CS point). Scanning through the space of parameters, it is possible to find the set of CS points. Finally, we plot the frontier for each given value of $\gamma$, in the $(\epsilon, \alpha)$ space.

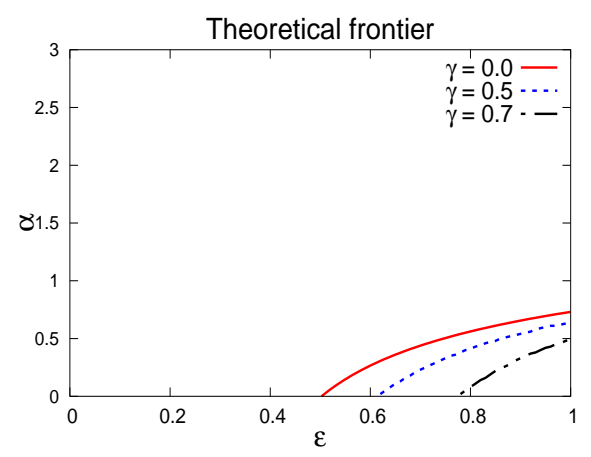

Figure 3.8: CS theoretical frontiers for different values of $\gamma$. The CS states, in each case, are located below the corresponding curve.

Figure 3.8 shows three examples, $\gamma=0,0.5$ and 0.7 of the theoretical frontier. The figure suggests that the advection plays a role that hampers CS states, shrinking the CS domain.

We also can find the theoretical limits for complete synchronization in the SAD model. Equation 3-31, defining $\eta=\epsilon \psi, \rho=\gamma \omega$ and $a=1-\epsilon$, can be transformed into

$$
(\eta+a)^{2}+\rho^{2} \leq\left(\frac{1}{2}\right)^{2}
$$

Geometrically, this corresponds to a circle. This implies $|\rho| \leq 1 / 2$, so

$$
\gamma|\omega| \leq \frac{1}{2}
$$

From Figure 3.7, for $\alpha=0$, we observe the maximal value $\left|\omega_{N-1}\right| \approx 0.63$. Then, equation (3-33) leads to the maximal value of $\gamma \simeq 0.79$ to observe synchronization, in accord with the results shown in Fig. 3.8.

\section{3}

\section{Delayed Purely Diffusive (DPD)}

We saw in (2-12) that the scheme with delay and diffusive coupling leads to the CML

$$
x_{t+1}^{i}=(1-\epsilon) f\left(x_{t}^{i}\right)+\frac{\epsilon}{\eta} \sum_{j=1}^{M} \frac{f\left(\hat{x}_{t}^{i+j}\right)+f\left(\hat{x}_{t}^{i-j}\right)}{r_{i, j}^{\alpha}},
$$

where $\hat{x}_{t}^{i}$ was defined in Chapter 2 as

$$
\hat{x}_{t}^{i}=\beta x_{t-1}^{i}+(1-\beta) x_{t}^{i} .
$$


In this case, due to delay, one can not obtain an equation like (3-2), then chaotic synchronization is unlikely. But still, we can consider regular orbits. For instance, if CS occurs at a fixed point, $x^{\star}$, such that

$$
x_{t}^{i}=x_{t-1}^{i}=x^{\star}
$$

and

$$
\hat{x}_{t}^{i}=\beta x_{t-1}^{i}+(1-\beta) x_{t}^{i}=x^{\star} .
$$

Then, the system of equations (3-34) becomes

$$
\hat{x^{\star}}=f\left(x^{\star}\right),
$$

which means that $x^{\star}$ needs to be a fixed point of the local dynamics.

Now, when linearizing equation (3-34) around the fixed point [21], we get

$$
\delta x_{t+1}^{i}=\left[(1-\epsilon) \delta x_{t}^{i}+\frac{\epsilon}{\eta} \sum_{j=1}^{M} A(r)\left(\delta \hat{x}_{t}^{i+j}+\delta \hat{x}_{t}^{i-j}\right)\right] f^{\prime}\left(x^{\star}\right) .
$$

The system of equations in (3-39) can be written in the form

$$
\delta \tilde{\mathbf{x}}_{t+1}=\mathbf{F}^{0} \delta \tilde{\mathbf{x}}_{t},
$$

by defining the $2 N$-dimensional tangent vector

$$
\delta \tilde{\mathbf{x}}_{t}=\left(\delta \mathbf{x}_{t}, \delta \mathbf{x}_{t-1}\right)^{T},
$$

and the $2 N \times 2 N$ time independent matrix,

$$
F^{0}=\left[\begin{array}{cc}
f^{\prime}\left(x^{\star}\right)[(1-\epsilon) \mathbb{1}+(1-\beta) \epsilon A] & f^{\prime}\left(x^{\star}\right) \beta \epsilon A \\
\mathbb{1} & 0
\end{array}\right]
$$

If we define $\mathbb{H}=[(1-\epsilon) \mathbb{1}+(1-\beta) \epsilon A]$ and $\mathbb{G}=\beta \epsilon A$, we can rewrite $F^{0}$ as

$$
F^{0}=\left[\begin{array}{cc}
\mathbb{H} f^{\prime}\left(x^{\star}\right) & \mathbb{G} f^{\prime}\left(x^{\star}\right) \\
\mathbb{1} & 0
\end{array}\right] .
$$

We can find the eigenvalues $\lambda$ of this matrix solving the equation $\operatorname{det}\left(F^{0}-\lambda \mathbb{1}\right)=0$. This implies that

$$
-\lambda \mathbb{1}\left[\mathbb{H} f^{\prime}\left(x^{\star}\right)-\lambda \mathbb{1}\right]-\mathbb{G} f^{\prime}\left(x^{\star}\right)=0,
$$

or

$$
\lambda^{2} \mathbb{1}-\lambda \uplus f^{\prime}\left(x^{\star}\right)-\mathbb{G} f^{\prime}\left(x^{\star}\right)=0 .
$$

On one hand, the two matrices, $\mathbb{H}$ and $\mathbb{G}$ depend on $A$. We recall that $A$ can be diagonalized in the Fourier base, therefore from (3-44) 


$$
\lambda^{2} \mathbb{1}-f^{\prime}\left(x^{\star}\right) \lambda[(1-\epsilon)+\epsilon(1-\beta) \Psi]-f^{\prime}\left(x^{\star}\right) \epsilon \beta \Psi=0 .
$$

Now, for each eigenvalue $k=0, \ldots, N-1$, the equation becomes

$$
\lambda^{2}-f^{\prime}\left(x^{\star}\right) \lambda\left[(1-\epsilon)+\epsilon(1-\beta) \psi_{k}\right]-f^{\prime}\left(x^{\star}\right) \epsilon \beta \psi_{k}=0 .
$$

This is the characteristic equation for $\lambda$. For each $k$ we have two values of $\lambda$.

On the other hand, the local map $f$ has two fixed points: $\{0,3 / 4\}$. Both are unstable in the uncoupled map, that is $\left|f^{\prime}\left(x^{\star}\right)\right|>1$, leading to chaos. However, their stability can change when the maps are coupled.

For $x^{\star}=0, f^{\prime}\left(x^{\star}=0\right)=4$. Then (3-46) becomes

$$
\lambda^{2}=-4 \lambda\left[(1-\epsilon)+\epsilon(1-\beta) \psi_{k}\right]-4 \epsilon \beta \psi_{k}=0 .
$$

It is easy to show, using the eigenvalues with $k=0$, that this fixed point remains longitudinally unstable.

For the second fixed point, $f^{\prime}\left(x^{\star}=3 / 4\right)=-2$. Then (3-46) becomes

$$
\lambda^{2}=2 \lambda\left[(1-\epsilon)+\epsilon(1-\beta) \psi_{k}\right]+2 \epsilon \beta \psi_{k}=0 .
$$

Using $\psi_{0}=\psi_{\max }=1$, equation (3-48) becomes

$$
\lambda^{2}+2 \lambda[1-\epsilon \beta]+2 \epsilon \beta=0
$$

Defining $\varphi \equiv \epsilon \beta$, the eigenvalues are

$$
\lambda^{ \pm}=-[1-\varphi] \pm \sqrt{[1-\varphi]^{2}-2 \varphi} .
$$

If we define the discriminant $\Delta=[1-\varphi]^{2}-2 \varphi$, we have two cases: $\Delta \geq 0$ and $\Delta<0$.

When $\Delta<0$, we have complex roots. In that case, $\left|\lambda^{ \pm}\right|<1$ becomes $\epsilon \beta=\varphi \leq 1 / 2$. When $\Delta \geq 0$, we have real roots. In that case, from $\left|\lambda^{ \pm}\right|<1$ we get $\epsilon \beta=\varphi \geq 1 / 4$.

Putting these results together

$$
\frac{1}{4} \leq \beta \epsilon \leq \frac{1}{2}
$$

In this interval the fixed point is longitudinally stable, along the synchronization subspace, differently to what happens with the other fixed point.

From the fact that $\epsilon \in[0,1]$, equation (3-51) implies that when we change $\beta$, the interval in $\epsilon$ for stability changes too. It is larger when $\beta=1 / 2$ and shorter for other values of $\beta$. For instance when $\beta=1 / 4, \epsilon=1$. If $\beta=1 / 2$, $1 / 2 \leq \epsilon \leq 1$. Now, when $\beta=3 / 4,1 / 3 \leq \epsilon \leq 2 / 3$. And if $\beta=7 / 8$, $2 / 7 \leq \epsilon \leq 4 / 7$.

For other eigenvalues, from equation (3-46), 


$$
\lambda^{ \pm}=-[1-\epsilon+\epsilon(1-\beta) \psi] \pm \sqrt{[1-\epsilon+\epsilon(1-\beta) \psi]^{2}-2 \epsilon \beta \psi}
$$

Defining $\Gamma \equiv[1-\epsilon+\epsilon(1-\beta) \psi]$ and the discriminant $\Delta \equiv \Gamma^{2}-2 \epsilon \beta \psi$, the roots are,

$$
\lambda^{ \pm}=-\Gamma \pm \sqrt{\Delta}
$$

Since $\Gamma^{2} \geq 0$, and $\psi_{\min }<0$, then $\Delta \geq 0$. So, for $\lambda^{+}$

$$
-\Gamma+\sqrt{\Gamma^{2}-2 \epsilon \beta \psi_{\min }}<1
$$

then

For $\lambda^{-}$, we obtain

$$
\epsilon \leq \frac{3}{2\left(1-\psi_{\min }\right)}
$$

$$
\Gamma+\sqrt{\Gamma^{2}-2 \epsilon \beta \psi_{\min }}<1
$$

from where

$$
\frac{1}{2\left[1-(1-2 \beta) \psi_{\min }\right]} \leq \epsilon
$$

putting together these two inequalities,

$$
\frac{1}{2\left[1-(1-2 \beta) \psi_{\text {min }}\right]} \leq \epsilon \leq \frac{3}{2\left(1-\psi_{\min }\right)} .
$$

This result gives the transversal stability boundaries for the complete synchronization domain of period 1 .

From equation (3-46) the stability conditions arise: longitudinal (3-51) and transversal (3-58). Both must hold for the existence of CS states in a fixed point. These results are coherent with those in [20, 21]. Now we can compare the results obtained for the two purely diffusive schemes (synchronous and delayed).

In Figure 3.9 theoretical results for the purely diffusive scheme, synchronous and delayed are shown. The small region enclosed by the continuous line, SDS case, contains only chaotic CS states as was shown in section 3.1. The CSR for the DPD model is enclosed by the active side of the inequalities found in equations (3-51) and (3-58). The longitudinal stability, blue dotted line, sets the right boundary of the CSR. The transversal stability, green dotted line, sets the left boundary. Enclosed between these two curves, we have completely synchronizes states of period 1 , for long range and short range of interactions. This illustrates the profound differences that emerge with the inclusion of delay. For the DPD model, the enclosed region is a subset of the CSR, including only 


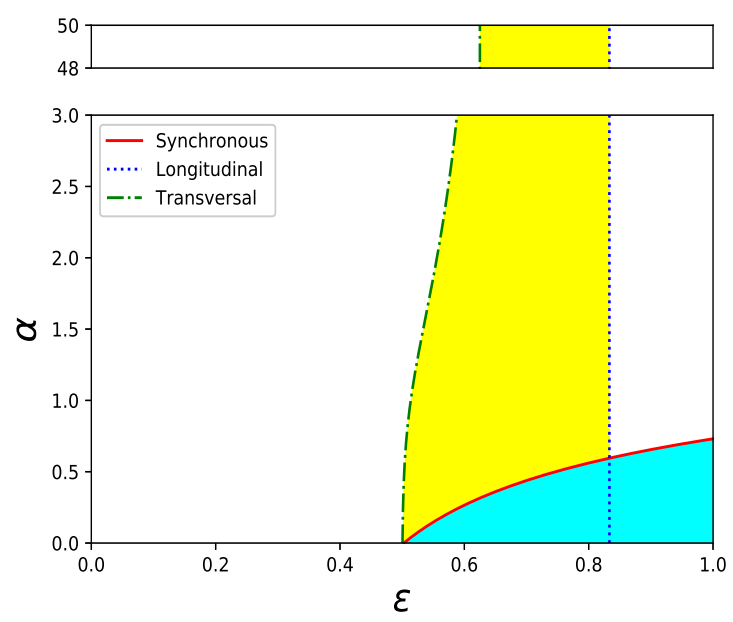

Figure 3.9: Theoretical CSR for the DPD, with $\beta=0.6$, and SPD models. The red (continuous) line indicates the frontier for the CSR of the synchronous model. The colored region below the curve corresponds to CS states for the SPD model. The green and blue lines (dotted) enclose the CSR for the delayed model, that includes short-range interactions, shown in the upper panel.

orbits of period 1. The simulations will give us the complete CSR that will include, as we will see, other types of orbits.

\section{4}

\section{Delayed Advective Diffusive (DAD)}

In the scheme with delay, diffusion and advection, the evolution of states is given by

$$
x_{t+1}^{i}=(1-\epsilon) f\left(x_{t}^{i}\right)+\frac{\epsilon}{\eta} \sum_{j=1}^{M} \frac{f\left(\hat{x}_{t}^{i+j}\right)+f\left(\hat{x}_{t}^{i-j}\right)}{r_{i, j}^{\alpha}}+\frac{\gamma}{\eta} \sum_{j=1}^{M} \frac{f\left(\hat{x}_{t}^{i+j}\right)-f\left(\hat{x}_{t}^{i-j}\right)}{r_{i, j}^{\alpha}} .
$$

In this case synchronization around a fixed point $x^{*}$, as in the DPD model, is also possible. Following the same procedure as before to calculate deviations around the fixed point, we get for the diffusive term

$$
\delta D=\epsilon(1-\beta) A f^{\prime}\left(x^{*}\right) \delta x_{t}+\epsilon \beta A f^{\prime}\left(x^{*}\right) \delta x_{t-1},
$$

and for the advective term

$$
\delta V=\gamma(1-\beta) B f^{\prime}\left(x^{*}\right) \delta x_{t}+\gamma \beta B f^{\prime}\left(x^{*}\right) \delta x_{t-1} .
$$

where we use the results from the previous sections. So, the evolution of displacements in the vicinity of the fixed point $x^{\star}$ becomes 
$\delta\left[x_{t+1}\right]=[(1-\epsilon) \mathbb{1}+\epsilon(1-\beta) A+\gamma(1-\beta) B] f^{\prime}\left(x^{*}\right) \delta x_{t}+[\epsilon \beta A+\gamma \beta B] f^{\prime}\left(x^{*}\right) \delta x_{t-1}$

Now we define

$$
\mathbb{H} \equiv \nVdash(\mathbb{N}, \alpha, \epsilon, \beta, \gamma)=(1-\epsilon) \mathbb{1}+\epsilon(1-\beta) A+\gamma(1-\beta) B,
$$

and

$$
\mathbb{G} \equiv \mathbb{G}(\mathbb{N}, \alpha, \epsilon, \beta, \gamma)=\epsilon \beta A+\gamma \beta B
$$

so equation (3-62) turns into

$$
\delta\left[x_{t+1}\right]=\mathbb{H} f^{\prime}\left(x^{*}\right) \delta x_{t}+\mathbb{G} f^{\prime}\left(x^{*}\right) \delta x_{t-1},
$$

that in matrix form is

$$
\left[\begin{array}{c}
\delta x_{t+1} \\
\delta x_{t}
\end{array}\right]=\left[\begin{array}{cc}
H f^{\prime} & \mathbb{G} f^{\prime} \\
\mathbb{1} & 0
\end{array}\right]\left[\begin{array}{c}
\delta x_{t} \\
\delta x_{t-1}
\end{array}\right] .
$$

The characteristic equation for this system, when $\lambda \neq 0$, is

$$
\lambda^{2} \mathbb{1}+2 \lambda \nVdash+2 \mathbb{G}=0,
$$

that in the Fourier base becomes

$$
\lambda^{2}+2 \lambda[(1-\epsilon)+\epsilon(1-\beta) \Psi-i \gamma(1-\beta) \Omega]+2[\epsilon \beta \Psi-i \gamma \beta \Omega]=0,
$$

and for each $k$

$$
\lambda^{2}+2 \lambda\left[(1-\epsilon)+\epsilon(1-\beta) \psi_{k}-i \gamma(1-\beta) \omega_{k}\right]+2\left[\epsilon \beta \psi_{k}-i \gamma \beta \omega_{k}\right]=0 .
$$

In equation (3-68), whenever the advection parameter $\gamma$ appears, it is accompanied by the delay parameter $\beta$. Then, both contributions can not be separated. One of these terms is proportional to $(1-\beta)$ and the other $\beta$.

Defining $\epsilon \phi \equiv \epsilon \psi-i \gamma \omega$ the equation becomes,

$$
\lambda^{2}+2 \lambda[(1-\epsilon)+\epsilon(1-\beta) \phi]+2[\epsilon \beta \phi]=0,
$$

which is very similar to the characteristic equation for the model without advection. So, if $\epsilon \gg \gamma$, this model converges to the previous one. In other cases, to find analytical solutions is not simple and has to be done for specific states.

For fixed values of $\beta$ and $\gamma$, we will look for couples of values $(\alpha, \epsilon)$ that make the corresponding two values of $\lambda$ in (3-69) holds $|\lambda| \leq 1$. If a state fulfills 

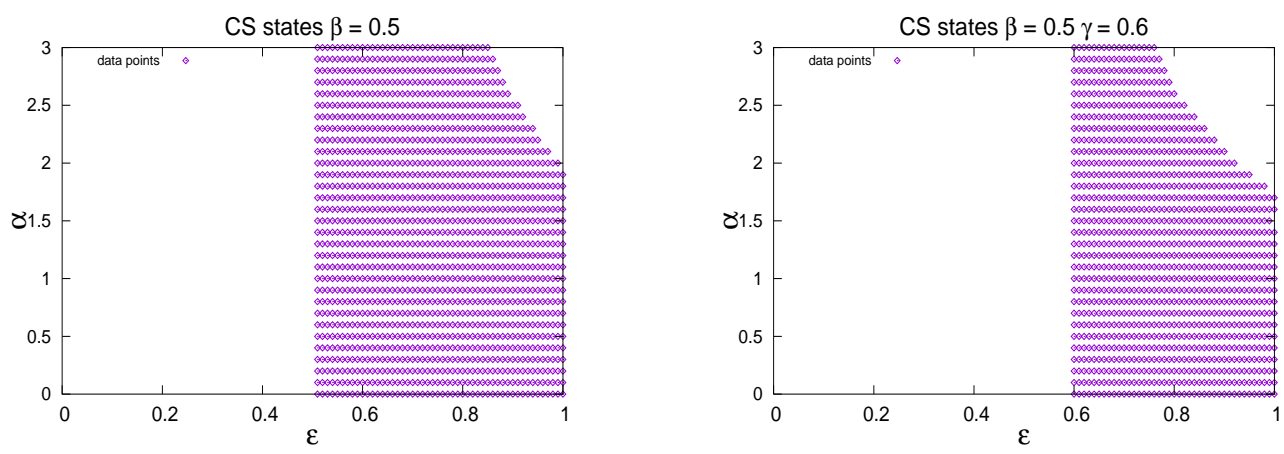

3.10(a): CS states for $\beta=0.5$ and $\gamma=0.0$.

3.10(b): CS states for $\beta=0.5$ and $\gamma=0.6$.

Figure 3.10: CSR for two illustrative cases of the delayed scheme, with and without advection.

both requirements, we mark the state as CS. Moreover, in the DAD model we also have to require the inequality $\epsilon \geq \gamma$.

In Figure 3.10, the comparison between (a) and (b) shows that advection primarily destroys CS states reducing the CS domain, due to the restriction $\epsilon \geq \gamma$. Second, for a fixed $\beta$, the advection destroys the CS states, as can be seen in the upper right corner of Figure 3.10(b). For fixed $\gamma$ the delay have the same effect as in the DPD model. 


\section{4}

\section{Numerical Results}

\section{1}

\section{Simulations setup}

For each scheme, numerical simulations were performed. Starting from random initial conditions [27], and a fixed set of values of the parameters $(\alpha, \epsilon, \beta, \gamma)$, we let the system evolve during a transient time $t_{0}=10^{4}$. After this time, we record the state of the system during the next 200 iterations. The size of the system is $N=201$ for all results shown.

Regarding the values of the parameters, we varied $\alpha \in[0,3]$ with a step of $\Delta \alpha=0.1, \epsilon \in[0,1]$ with $\Delta \epsilon=0.01, \gamma \in[0,1]$ with step of $\Delta \gamma=0.1$ and $\beta \in[0,1]$ with step of $\Delta \beta=0.1$. Other specific values of parameters were also considered.

\section{2}

\section{Collective metrics}

As in $[20,21]$, we calculate collective metrics to measure the global behavior of the system. We consider the average state,

$$
h_{t}=\frac{1}{N} \sum_{i=1}^{N} x_{t}^{i}
$$

and its standard deviation,

$$
\sigma_{t}=\sqrt{\frac{1}{N} \sum_{i=1}^{N}\left(x_{t}^{i}-h_{t}\right)^{2}} .
$$

Notice that for a completely synchronized state, as defined before, it should be $\sigma=0$. In practical terms we consider CS when $\sigma<10^{-14}$. Also, as in $[20,21]$, we calculate the largest Lyapunov exponent $\lambda_{t}$ using the Benettin algorithm [28]. 


\section{3}

\section{Results for the Synchronous Purely Diffusive model}

Following the procedure described above, we calculate $h, \sigma$ and $\lambda$ for the SPD model.

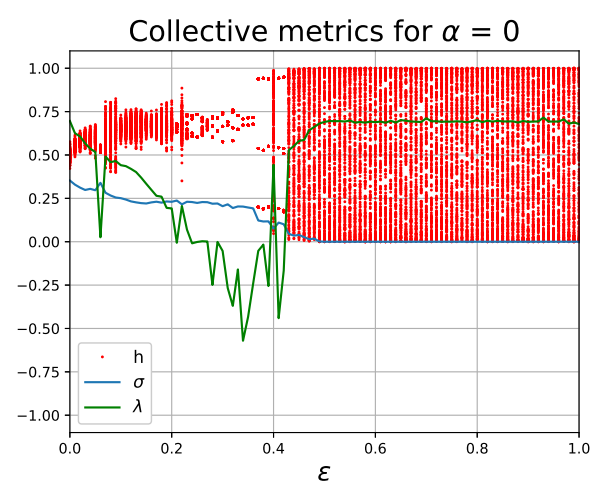

4.1(a): CS occurs for $\epsilon \geq 0.5$. The density of the orbits and the positive $\lambda$ indicate that the CS states are chaotic.

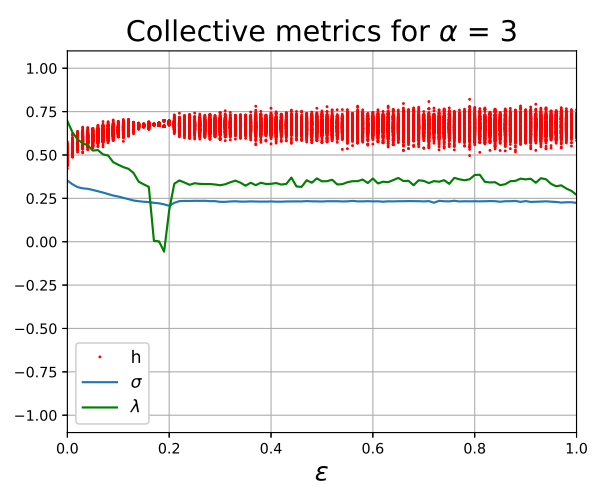

4.1(b): For range of interactions $\alpha \geq 1$, the synchronous schemes do not have CS states, regardless $\epsilon$.

Figure 4.1: Collective metrics for $\alpha=0$ and $\alpha=3$. The red dots, representing $h_{t}$, correspond to 200 consecutive values after the transient $t_{0}$.

In Figure 4.1(a), corresponding to fixed $\alpha=0$, complete synchronization $(\sigma=0)$, occurs for $\epsilon \geq 0.5$, and in Figure 4.1(b), corresponding to fixed $\alpha=3$, complete synchronization does not occur for any value of the parameter $\epsilon$ ( $\sigma$ is always positive). This is because complete synchronization is limited in the SPD model by the range of interactions, that needs to be long enough. Moreover, the positive Lyapunov exponent in the CS region, indicates chaos. All these results from simulations are in total agreement with the theoretical prediction obtained in the previous chapter, in equation (3-17) and Figure 3.4.

Considering the values of $\sigma$ and $\lambda$, in figure 4.1(a), we can distinct three regions.

- Region I: $\sigma$ and $\lambda$ positive.

- Region II: $\sigma$ positive and $\lambda$ negative.

- Region III: $\sigma=0$ and $\lambda$ positive.

Region I is characterized by no collective behavior, due to values of $\epsilon$ close to 0 , which makes the coupling too weak to produce a pattern. Region II is characterized by ordered states, as indicated by a negative $\lambda$, but no CS occurs in that region as is indicated by a positive $\sigma$. Region III corresponds to CS where $\sigma=0$. In this case, $\lambda$ is positive, indicating that CS states are chaotic. 
The values of $\sigma$ and $\lambda$ are indicators of certain types of underlying dynamics, but it is the average $h$ that shows the inner structure of the orbits. In Figure 4.1(a) for example, in Region II, $\lambda<0$ indicates that those states have periodic orbits but it is $h$ that shows the inner structure of the orbit, with more detail.

Now we look at these metrics in the whole parameter space $(\alpha, \epsilon)$.

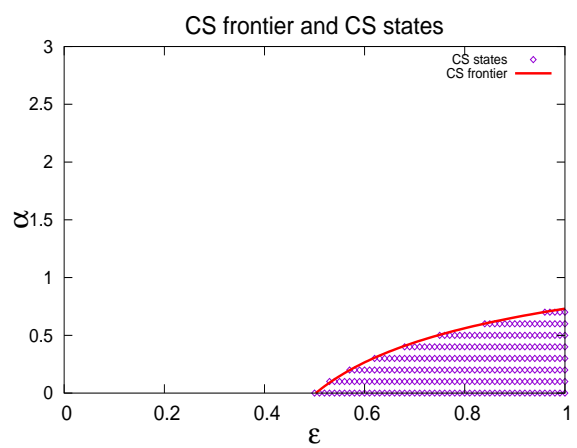

4.2(a): The red line corresponds to the theoretical frontier given by equation $(3-17)$ and the dots to the CS states with $\sigma \leq 10^{-14}$ obtained by numerical simulations.

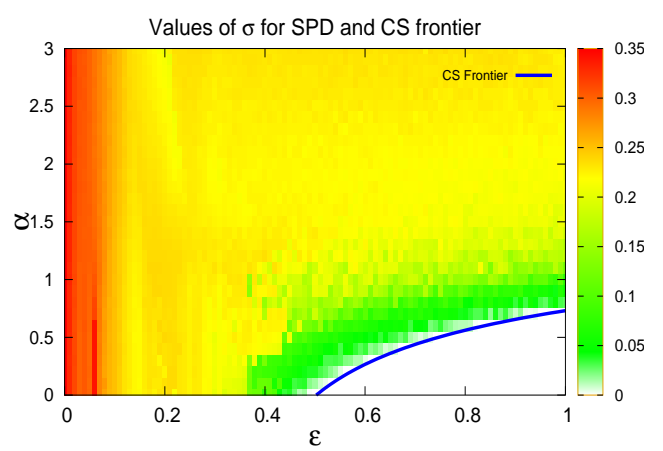

4.2(b): The values of $\sigma$ in $(\alpha, \epsilon)$ show four different regions. The CS states are shown in white and the theoretical frontier in blue.

Figure 4.2: CS states, frontier and $\sigma$ for the SPD model.

In Figure 4.2(a), the line corresponds to the theoretical frontier obtained in equation (3-17) and the dots correspond to results of simulations with $\sigma \leq 10^{-14}$. The states with CS are below the curve as was predicted in the previous chapter.

In Figure 4.2(b), the average value of $\sigma$ for each case $(\alpha, \epsilon)$ is shown. This heatmap has four distinctive regions. For $\epsilon<0.1$, maps are almost uncoupled, then $\sigma$ is high (red region). In the intermediate range $0.1 \leq \epsilon \leq 0.4$, the standard deviation $\sigma$ tends to a fixed value different from zero $(\sigma=$ $0.223 \pm 0.06$ ) (yellow region). Figure 4.1(b) is an example of this type of states. For strong coupling and not too long range (green region), the trajectories of individual maps are very close but synchronization is not complete. For strong coupling and sufficiently long range interactions, complete synchronization occurs (white region) and orbits are chaotic. Examples of these orbits can be found in the Appendix A. 
The results in parameter space for $\lambda$.

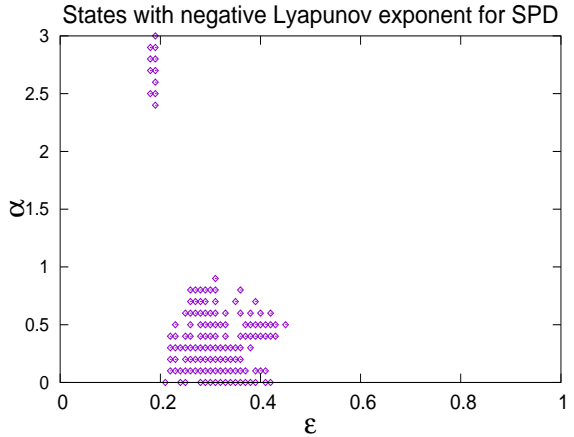

4.3(a): Simulations show two regions where $\lambda<0$, associated with regular orbits.

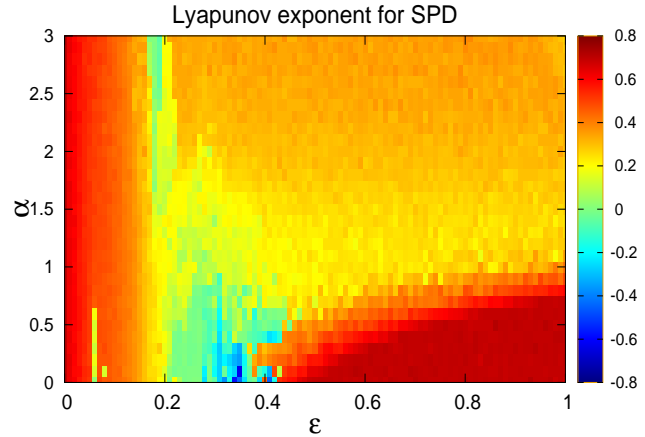

4.3(b): Values of $\lambda$ in the whole $(\alpha, \epsilon)$ space. As was predicted, the CS states have chaotic orbits, indicated by $\lambda>0$.

Figure 4.3: The Lyapunov exponent for the SPD model.

In Figure 4.3(a), the region where $\lambda<0$ is located approximately in $0.2 \leq \epsilon \leq 0.4$. These states correspond to regular orbits, although the system is not completely synchronized. In Figure 4.3(b), the higher values of $\lambda$ coincide with the region of CS shown in Figure 4.2 and predicted in the previous chapter. This is because in the SPD model, every state that is completely synchronized is chaotic. 


\section{4}

\section{Results for the Synchronous Advective Diffusive model}

For the SAD model, two illustrative examples of the collective metrics, for $\alpha=0$ are shown below.

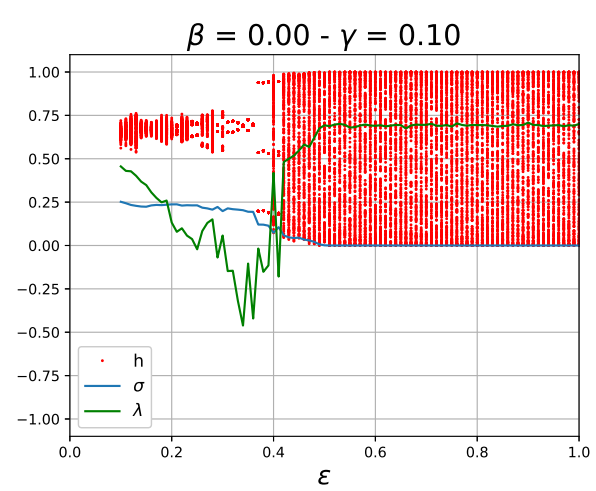

4.4(a): For $\alpha=0$ and $\gamma=0.1$, CS occurs for $\epsilon \geq 0.51$. The density of the orbits and the positive $\lambda$ indicate that the CS states are chaotic.

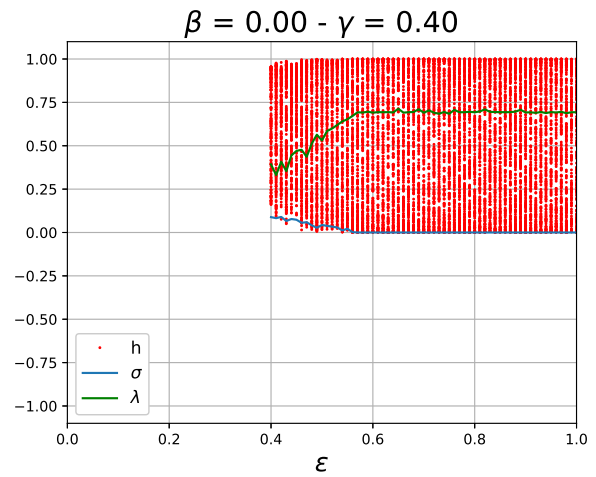

4.4(b): For $\alpha=0$ and $\gamma=0.4$, CS occurs for $\epsilon \geq 0.57$.

Figure 4.4: Collective metrics $h, \sigma$ and $\lambda$ for the SAD model.

In Figure 4.4 we can see that for $\alpha=0$, the SAD model has CS states. This region of complete chaotic synchronization is indicated by $\sigma=0, \lambda>0$ and dense orbits, in the sense that that with more iterations they will fill up the interval completely [22]. We also notice that increasing the contribution of advection, from $\gamma=0.1$ in (a) to $\gamma=0.4$ in (b), reduces the domain where $\sigma=0$ without changing the type of orbits that completely synchronize. Both results were previously founded in section 3.2 .
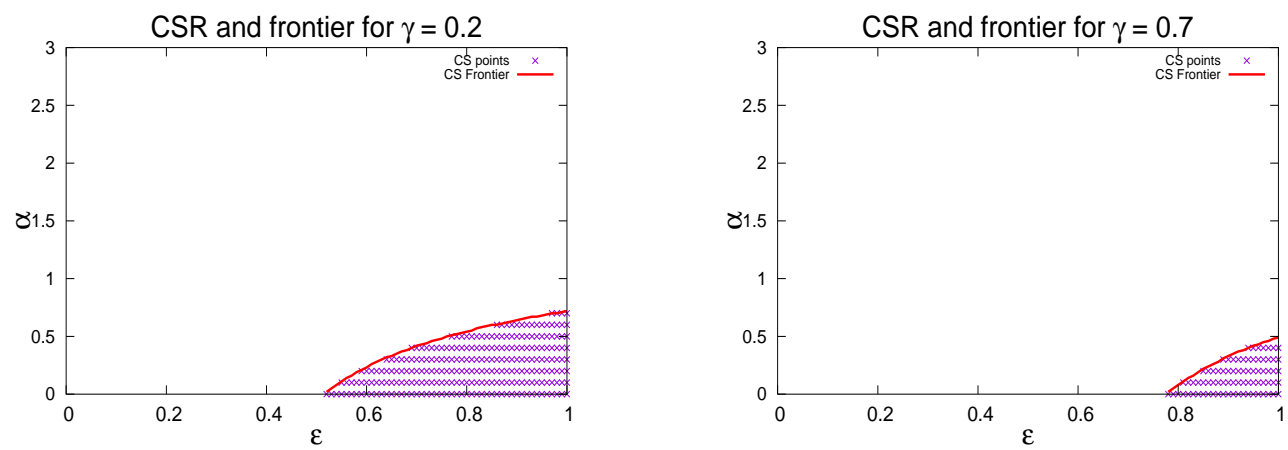

Figure 4.5: CS states (dots) and theoretical frontier (red line) for two $\gamma$ values of the SAD model show the reduction of the CSR.

In Figure 4.5 CS states from the simulation and the corresponding theoretical frontier are shown together. In (a), for $\gamma=0.2$ the CSR is similar to the one 
founded for the SPD model. In (b), for $\gamma=0.7$ the CSR has been reduced to a small fraction of the original. This indicates that increasing $\gamma$ reduces the number CS states, in concordance with what we found in section 3.2. This reduction process continues up to a critical value of $\gamma_{c} \approx 0.792$ that we found in simulations as a limit for the existence of the CSR. For $\gamma>0.792$, the system does not have CS states, as we already found in section 3.2.

Given a point in the space $(\epsilon, \alpha)$ within the CSR in the absence of advection $(\gamma=0)$, CS is conserved up to a critical value $\gamma_{c}$. Beyond that value, the effect of advection is to progressively separate or spread out the individual trajectories, as illustrated in Figure 4.6. To illustrate this phenomenon, we take individual trajectories from two cases of the SAD model. The individual maps are: $i \in[1,30,40,60,70,90,100,150,170,180,190,201]$. Starting with the longtime state for $(\alpha, \epsilon)=(0.7,0.98)$, which is completely synchronized without advection, we increase the value of $\gamma$ to put into evidence the effect, which is weak but visible for $\gamma>0.4>\gamma_{c}$.

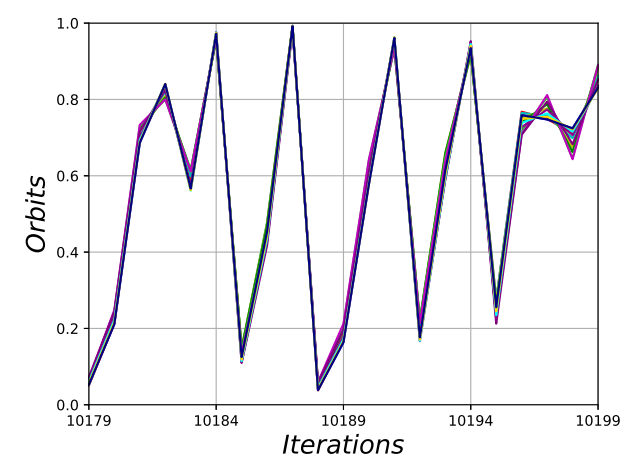

4.6(a): When $\gamma=0.4$ the spread out is observable and the CS state has been lost.

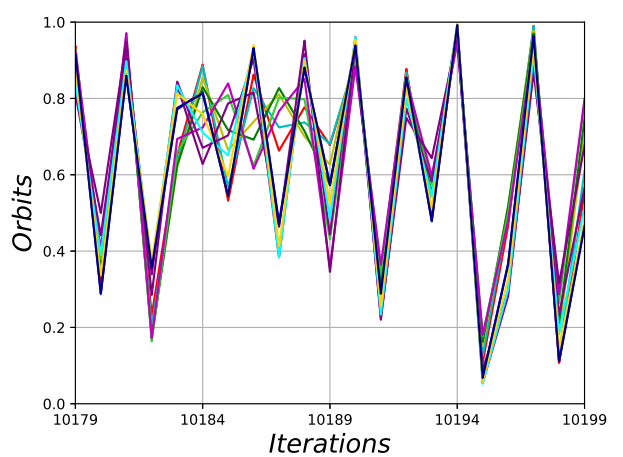

4.6(b): When $\gamma=0.8$ the spread becomes larger.

Figure 4.6: Effect of advection. For each case, the same 12 individual trajectories, for 20 iterations after the transient time, are shown.

Figure 4.7 shows the heatmaps of $\sigma$ and the largest Lyapunov exponents in the plane $(\epsilon, \alpha)$. In both heatmaps, the region $\epsilon<0.1$ is forbidden $(\epsilon \geq \gamma)$. This cuts off the red zone of $\sigma$ that appears in the SPD model in Figure 4.2(b). The white zone in Figure 4.7(a), where CS occur, will decrease with advection as we predicted in section 3.2. As for the Figure 4.7(b), increasing $\gamma$ will reduce the red zone associated with completely synchronized chaotic states. It will also reduce the number of states with negative Lyapunov exponent (NLE). 


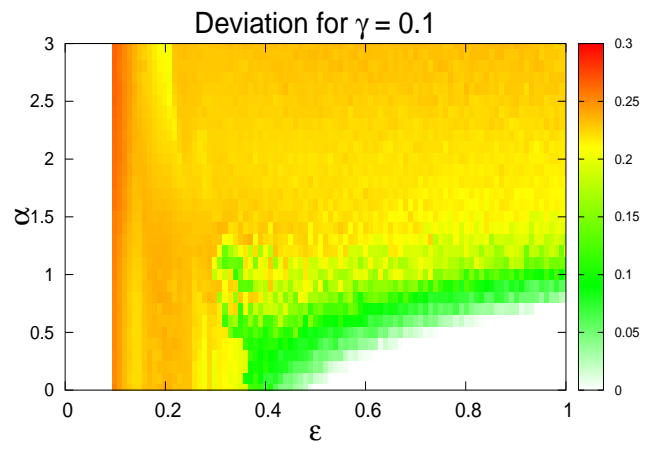

4.7(a): Average value of $\sigma$ for SAD model with $\gamma=0.1$

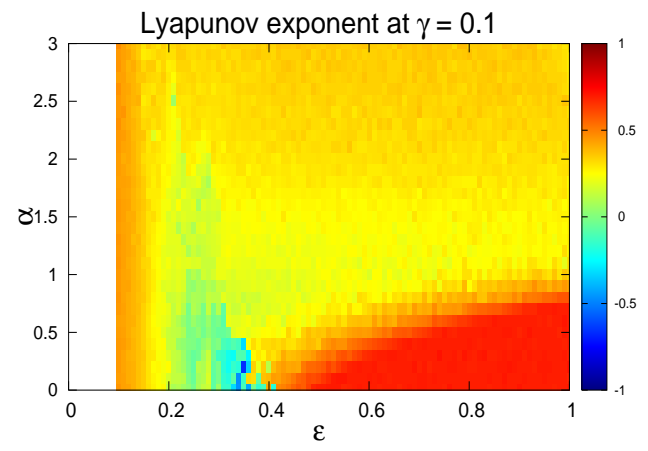

4.7(b): Average value of $\lambda$ for SAD model with $\gamma=0.1$

Figure 4.7: The heatmaps show average values in the parameter space. For low values of advection, they resemble those for SPD model.

In Figure 4.8(a), for $\gamma=0.1$, the upper zone in Figure 4.3(a) has completely disappeared and the lower region has been reduced. This shows the effect of $\gamma$ over NLE states. Now, when we set $\gamma=0.3$, as in Figure 4.8(b), the region becomes even smaller. At $\gamma=0.4$ there are no points with negative $\lambda$ in the whole parameter space. For $\gamma=0.5$ a new small region appears around $\epsilon=0.5$ and start moving to the right with increasing $\gamma$. Complementary results can be found in the Appendix B.
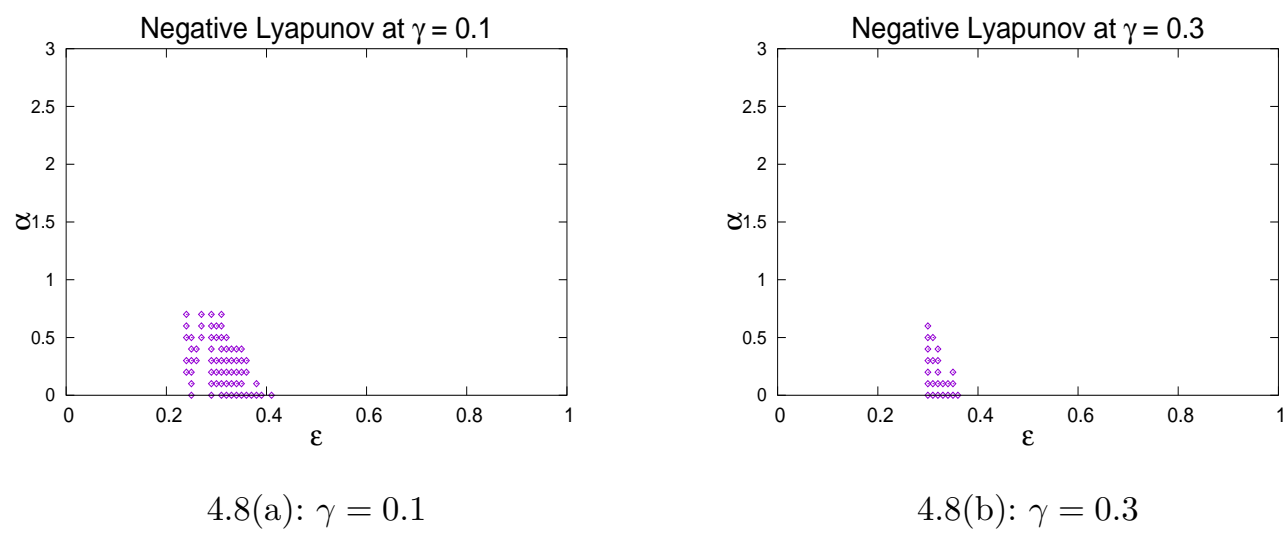

Figure 4.8: Simulations show that increasing $\gamma$ reduce the number of states with $\lambda<0$.

We can observe these effects over the individual trajectories. To do so, we use the same trajectories as before. Starting at $(\alpha, \epsilon)=(0,0.41)$ that is characterized by a NLE, we increase the value of $\gamma$. 
In Figure 4.9 we can see that the effect is similar with respect to that discussed above for CS. This shows a closer look to the global behavior evinced in sections 3.2 and 4.4 .

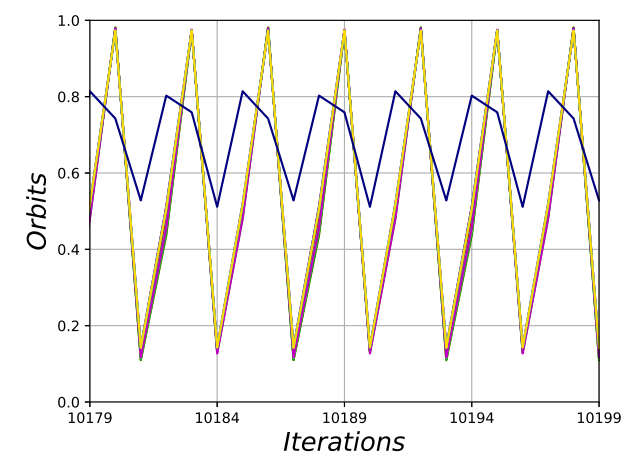

4.9(a): When $\gamma=0.1$ the trajectories start to spread out.

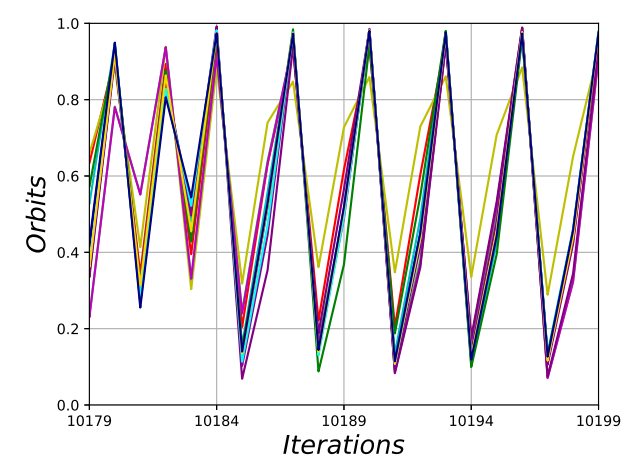

4.9(b): When $\gamma=0.3$ the state is not longer characterized by a NLE.

Figure 4.9: Effect of advection over a state with negative Lyapunov exponent. For each case, the same 12 individual trajectories, for 20 iterations after the transient time, are shown.

Furthermore, from this data, we can observe that the effect of the advection is incremental. For instance, the CSR does not disappear abruptly at some level of advection it shrinks progressively. The same occurs with the NLE states.

\section{5}

\section{Results for the Delayed Purely Diffusive model}

From the previous chapter, we expect a change in the dynamics once we include delay. Two examples of the results for the collective metrics, for two different values of the contribution of the delay, are shown below.

In Figure 4.10 the feature that stands out is the change in the CS states orbits. As found in section 3.3, the synchronized states are not necessarily chaotic. In fact, in (a), we have a mixture of different types of orbits in the interval where $\sigma=0$. Even more, none of those orbits is chaotic. Now, in (b), when $\beta=0.8$, the possibility of CS states $(\sigma=0)$ which are chaotic $(\lambda>0)$, has reappeared.

The comparison between Figure 4.10(a) with Figures 4.1 and 4.4 puts into evidence new features of the model with delay. In (a), the system is able to synchronize with weaker coupling, at $\epsilon=0.42$. Also in (a) there are 3 regions where $\lambda<0$ and in those intervals, $h$ shows periodic windows. For (b), in the large interval where $\lambda<0$, there is an orbit of period 1 for $\epsilon \in[0.51,0.62]$, followed by a window of period 3 . 


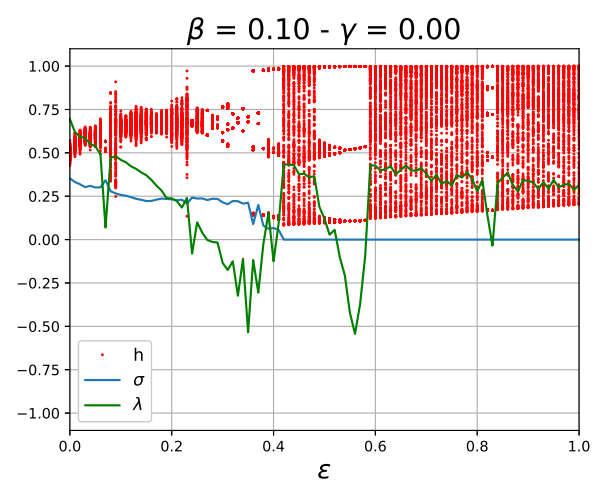

4.10(a): $\quad \alpha=0, \beta=0.1$

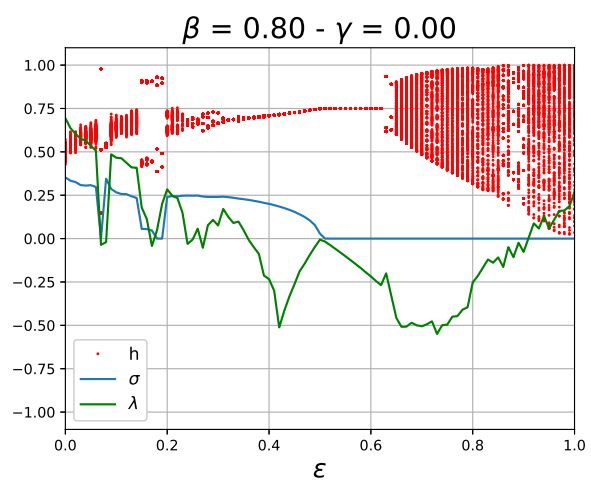

$4.10(\mathrm{~b}): \quad \alpha=0, \beta=0.8$

Figure 4.10: Collective metrics $h, \sigma$ and $\lambda$ at $\alpha=0$ for the DPD model. Simulations show that delay creates new types of orbits and change the CS states.

Simulations also show that the delayed scheme favors completely synchronized states, in comparison with the synchronous scheme, as was predicted in section 3.3 and depicted in Figure 3.9. Now, if we consider only the delayed scheme, the effect of increasing values of $\beta$ can be divided into two intervals. In the interval $0 \leq \beta \leq 0.5$, the CSR becomes larger with increasing $\beta$. From there, increasing $\beta$ shrinks the CSR. Even more, for $\epsilon \geq 0.8$ a new region of CS emerges in the interval $\epsilon \in[0.15,0.2]$, as shown in Figure 4.11(b). This was not predicted in section 3.3 because these CS state are not associated with the fixed point $x^{*}=3 / 4$. All these results are coherent with those in [21].

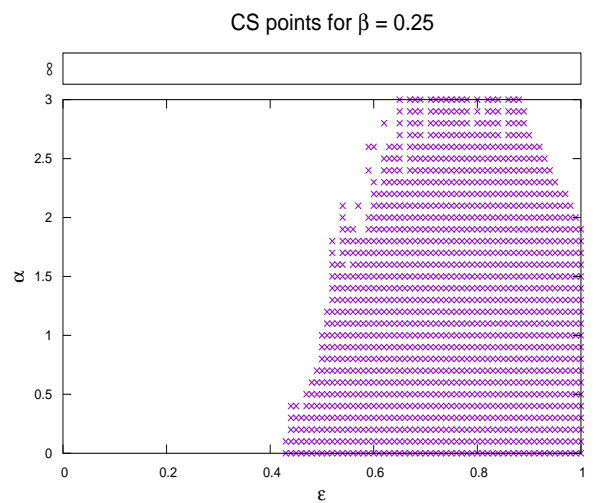

4.11(a): For $\beta=0.25$ the CSR is clearly larger than the CSR for the SPD model.

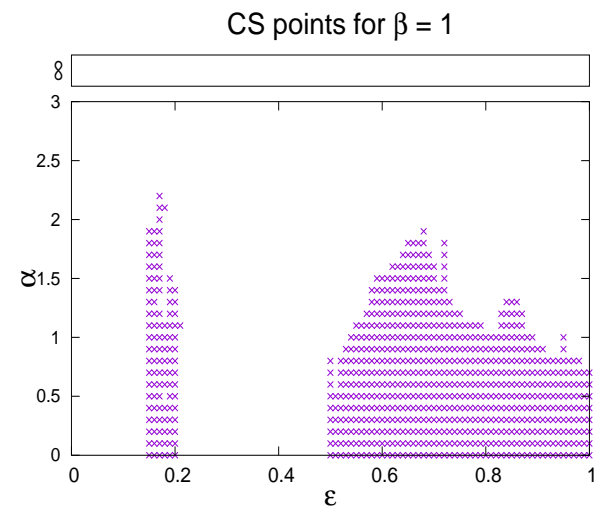

4.11(b): For $\beta=1$ the CSR is also larger that the one for the SPD model.

Figure 4.11: Complete synchronization states for DPD model.

The delay displays similar effect in the states whose Lyapunov exponent is negative, as can be seen in Figure 4.12. 


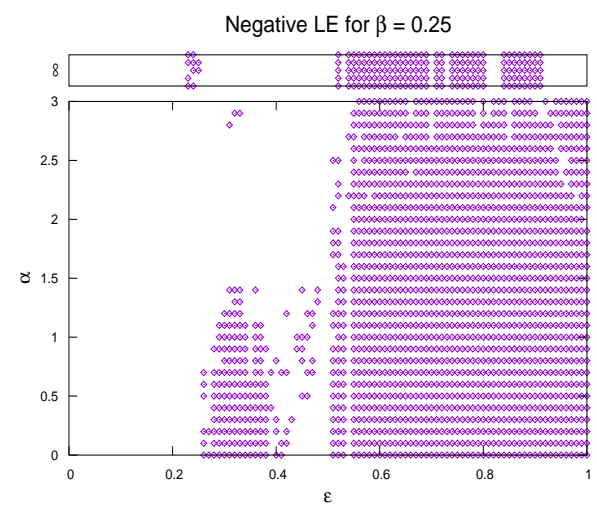

4.12(a): Small delay favors states with negative $\lambda$.

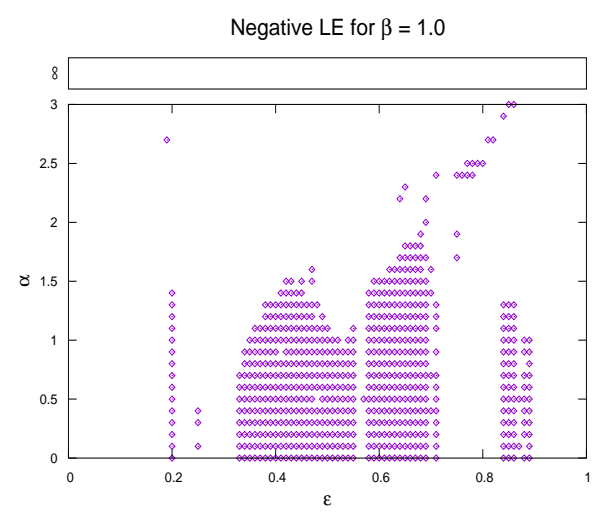

4.12(b): With large $\beta$ the number of NLE states decreases.

Figure 4.12: Negative Lyapunov exponent for the DPD model.

In Figure 4.12, the NLE states obtained from simulations are shown. The results indicate that the number of this type of states increases up to a $\beta=0.4$ and then, with larger delay, the number falls, in a way, showing a similar trend as the CS states. The difference here is that the value of $\beta$ is not the same. For CS states, is $\beta=0.5$, while for NLE states is $\beta=0.4$.

One distinctive feature that appears with the inclusion of delay is the emergence of regular orbits. This starts with a cascade of orbits of period $2^{k}$, from right $(\epsilon=1)$ to the left $(\epsilon=0)$, as illustrated in figure 4.13.

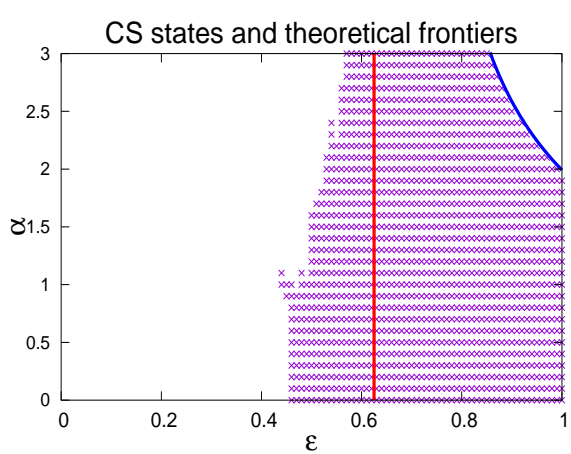

4.13(a): The dots correspond to the CS states. Inside the solid lines, orbits of period 1 are completely synchronized.

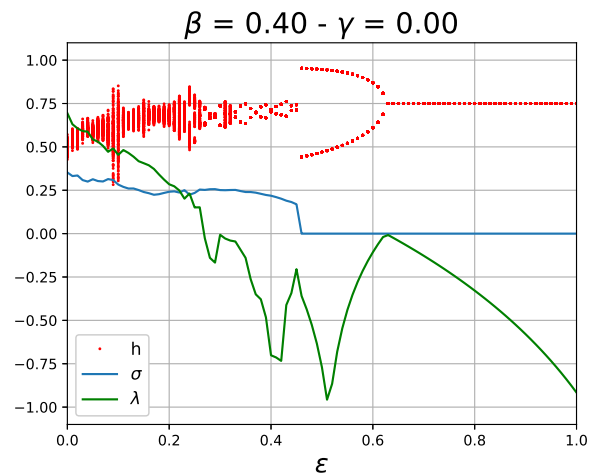

4.13(b): The value of $h$ show the type of orbit that are contained in the CSR in (a).

Figure 4.13: For $\beta=0.4, \alpha=0$ the CS states, frontiers and collective metrics.

A second distinctive feature is that there is complete synchronization of these regular orbits. This type of synchronization did not emerge in the synchronous scheme, where the CS states are only chaotic. In Figure 4.13(a), the CS states are composed only by regular orbits. The CS states of period 1 
can be identified because they are located between the solid lines that represent the inequalities found in section 3.3. In panel (b), the value of $h$, for $\alpha=0$, shows these regular orbits more clearly. We also see that in that region, $\sigma=0$, i.e., the collective states are CS.

As a complementary example, we look at a higher value of the contribution of delay, $\beta=1$. In Figure 4.14, regular and chaotic orbits coexist in the CSR. In (a), the CSR is composed by two separated zones. In (b), the value of $h$ indicates the type of orbits that completely synchronize, where $\sigma=0$. As we can see, when $\beta$ is large enough, chaotic orbits emerge for $\epsilon>0.9$.

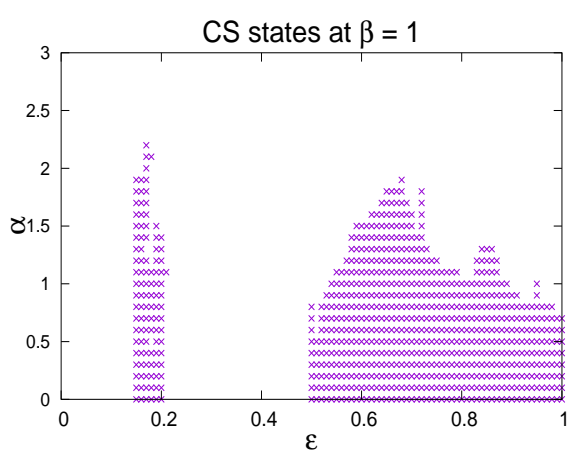

4.14(a): For $\beta$ large enough, the CSR is composed by two separated regions.

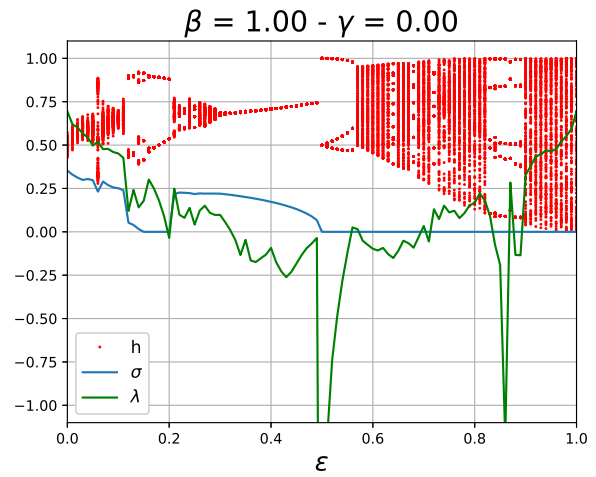

4.14(b): The value of $h$ show that the two CSR have contain regular orbits, but the one on the right can also have chaotic CS states.

Figure 4.14: For $\beta=1$ the CS states and the collective metrics. In this case $\alpha=0$.

As a result it is claimed that delay favors the formation of regular orbits and completely synchronized states. Therefore, we will have much more states with negative Lyapunov exponents. 
Besides the CS and NLE states, other features also change in comparison with the models with synchronous update scheme. This can be seen through heatmaps.

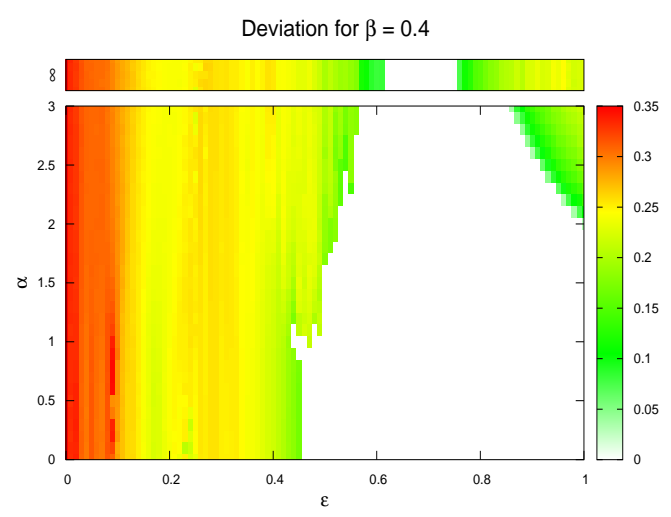

4.15(a): Average values of $\sigma$ for $\beta=0.4$

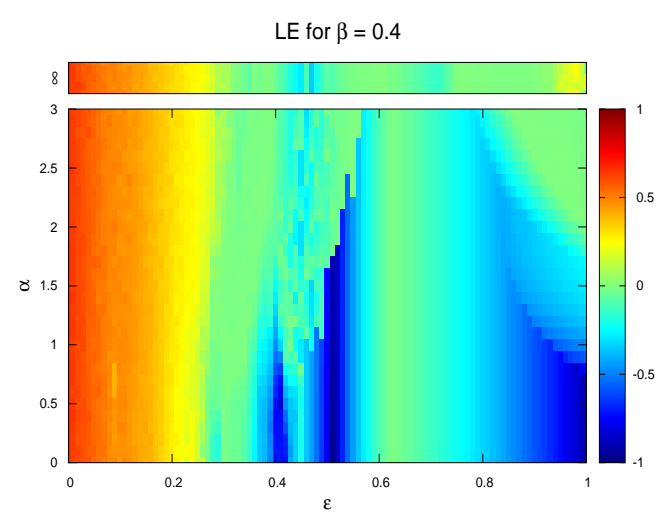

4.15(b): Average values of $\lambda$ for $\beta=0.4$

Figure 4.15: Deviation and Lyapunov exponent heatmaps for the DPD model when $\beta=0.4$.

Figure 4.15(a), when compared with Figure 4.2(b), shows that the red region, where $\epsilon<0.1$ does not change due to delay. All the other regions of the parameter space are altered. CS states, in white, now also occupy the short range interactions zone. Here, the green and yellow regions are more mixed in comparison with the SPD model. Now if we compare Figure 4.15(b) and $4.3(\mathrm{~b})$, we can immediately see that we have an extended region of regular orbits, approximately starting in $\epsilon \geq 0.2$, and also that some of these orbits coincide with the CS states shown in 4.15(a). An example of these orbits, was found in section 3.3. An example from simulations is shown in Fig. 4.16.

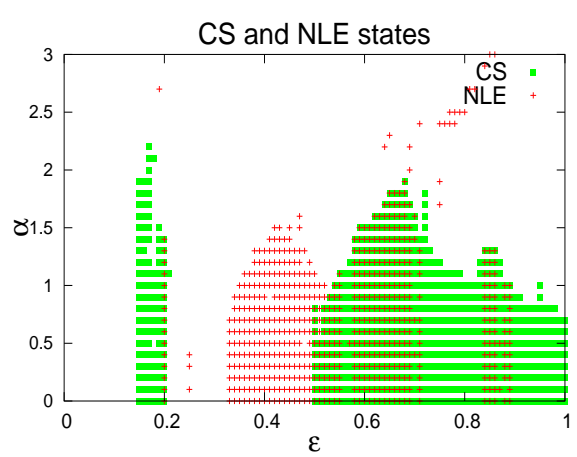

4.16(a): CS and NLE states together

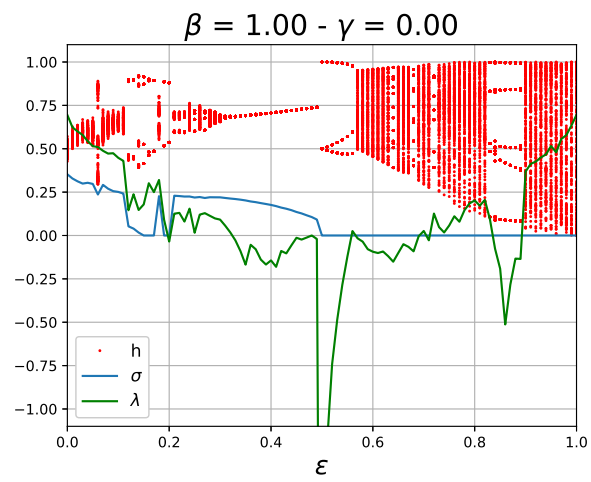

4.16(b): $\beta=1$ at $\alpha=0.5$

Figure 4.16: For $\beta=1$, the overlap of regular and CS states.

Complementary results can be found in the Appendix C. 


\section{6}

\section{Results for the Delayed Advective Diffusive model}

In this model, as well as in the SAD model, the coupling parameter $\epsilon$ can not be smaller than the advection parameter $\gamma$.

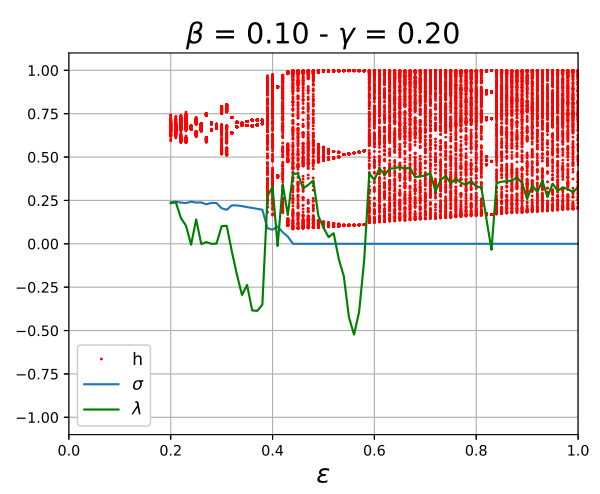

$4.17(\mathrm{a}): \quad \alpha=0, \beta=0.1, \gamma=0.2$

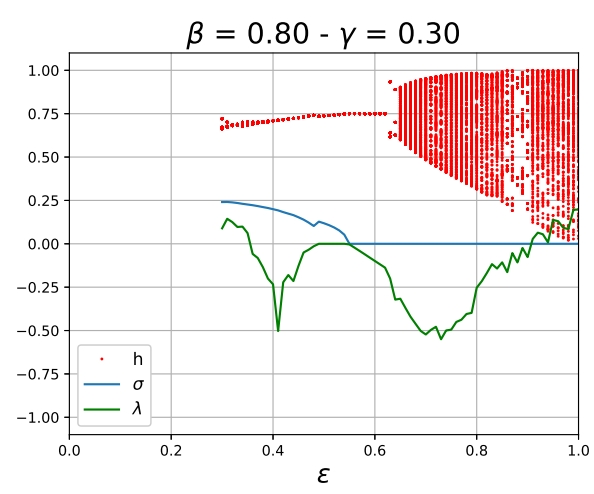

4.17(b): $\quad \alpha=0, \beta=0.8, \gamma=0.3$

Figure 4.17: The Collective metrics $h, \sigma$ and $\lambda$ for two illustrative examples in the DAD model.

When we compare the metrics in Figure 4.17 with those in Figure 4.10, it is possible to evidence the effects of advection in presence of delay. In (a), the minimal value of $\epsilon$ for which the system synchronizes has moved to $\epsilon=0.44$. For the same parameter, in (b), we have a shift to $\epsilon=0.55$. Moreover, in (a), the negative peak located around $\epsilon=0.36$ has moved upwards, and the average $h$ shows a new periodic window near $\epsilon=0.42$.

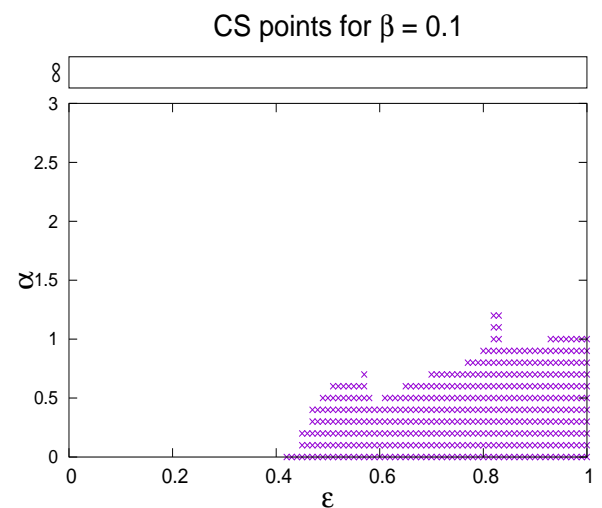

4.18(a): DPD case with $\beta=0.1$ and $\gamma=0$

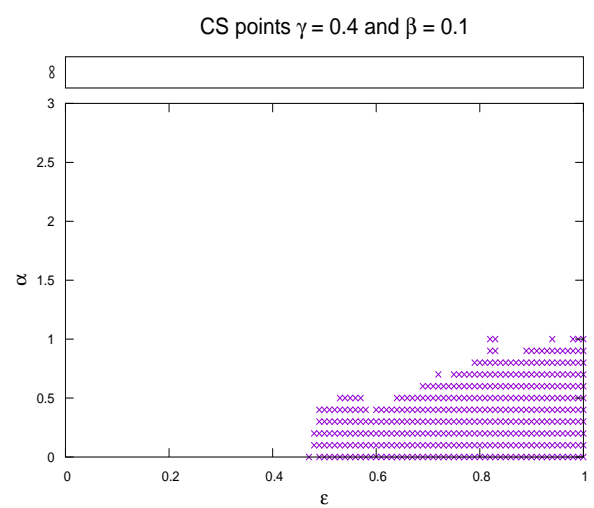

4.18(b): DAD case with $\beta=0.1$ and $\gamma=0.4$

Figure 4.18: The effect of advection when the delay parameter is fixed.

Now we focus on CS states. Figure 4.18 shows that the CS region deceases only slightly and remains almost unchanged, in size and location, 
with increasing advection. From the previous section we know that CS states grow with delay and advection will reduce the CS states. The reduction due to advection is almost negligible and the net effect is similar to the DPD model.

For stronger advection, $\gamma>0.5$, the $(\alpha, \epsilon)$-plane will be cut due to the restriction over $\epsilon$. In this sense, the CS region will decrease with advection, but mostly as a result of a change in the parameters space. When both parameters are above 0.5 , the observed combined effect is a decreasing number of CS states. The exception seems to be $\beta=0.5$, where the completely synchronized states remains unchanged for $\gamma \leq 0.5$.

Then, we can put forward the idea that delay overcomes advection. Neglecting some small deviation, the effect of advection over synchronized states, is weak. This is reflected in the number of CS states and some small deviation from the DPD model in the collective metrics.

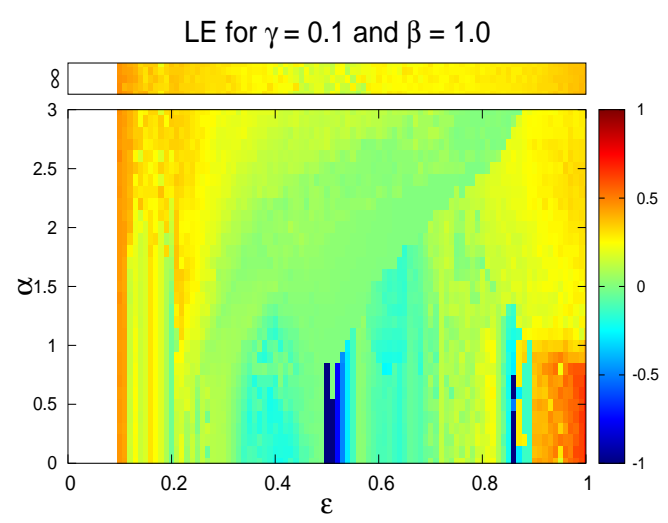

4.19(a): Lyapunov exponent

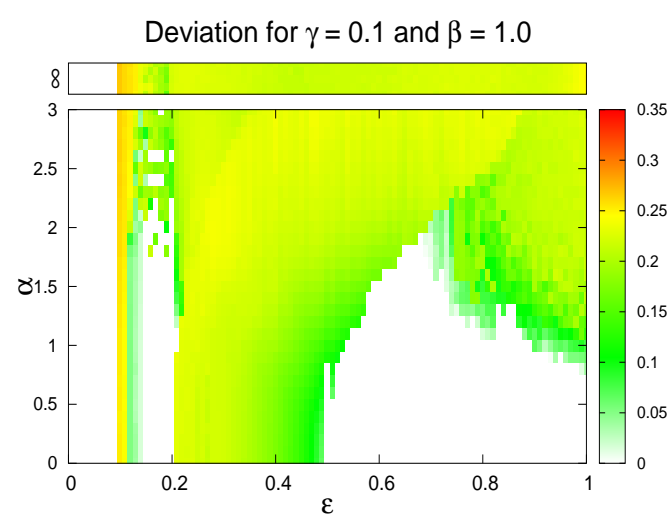

4.19(b): Deviation

Figure 4.19: Heatmaps for the DAD model.

The effect of the advection in other points is also dominated by the delay. For example, the deviation maintains almost the same portrait as the DPD model as shown in Figure 4.19.

As we know from the previous section, the effect of $\beta$ on the NLE region has two phases. In both of them, due to the extension of the region where the Lyapunov exponent is negative, the advection effect comes essentially from cutting the plane. 


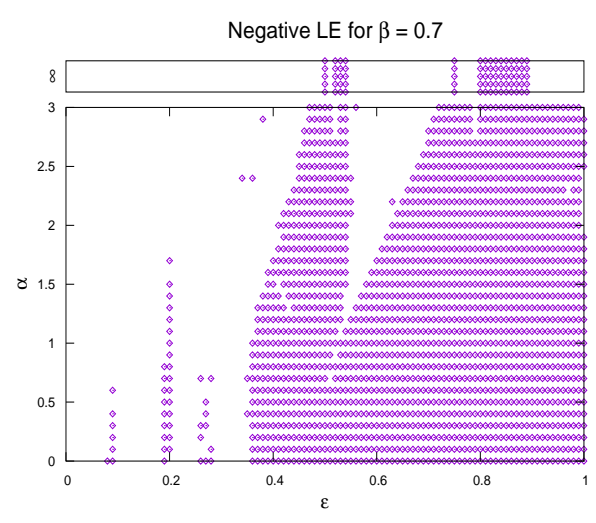

4.20(a): $\quad$ DPD case with $\beta=0.7$

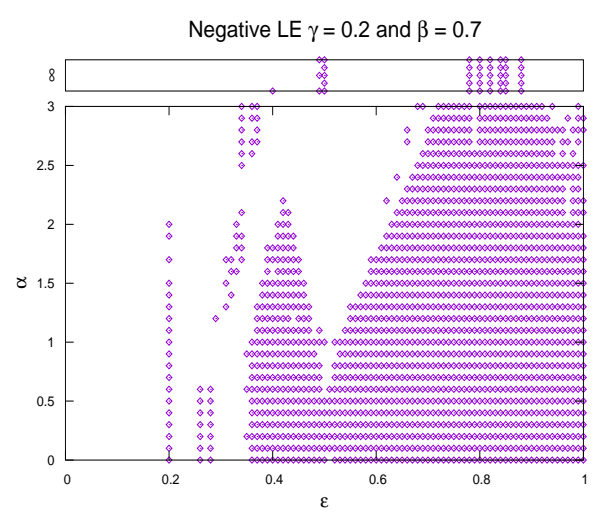

4.20(b): DAD case with $\beta=0.7$ and $\gamma=0.2$

Figure 4.20: Short-range sensitivity to advection.

As an example, Figure 4.20 shows that points in the short-range region change their $\lambda$ first. The changes that advection produces in these type of states are moderate compared with the synchronous version, where the NLE completely disappears for $\gamma=0.4$.

We can see that when advection is introduced in the delayed model, the effects over CS and negative Lyapunov exponent states are in the same direction as in the model without delay, but in this case, due to the delay, they are practically hidden. Complementary results can be found in the Appendix D.

We can visualize the combined effect of advection and delay comparing the collective metrics for some illustrative examples.

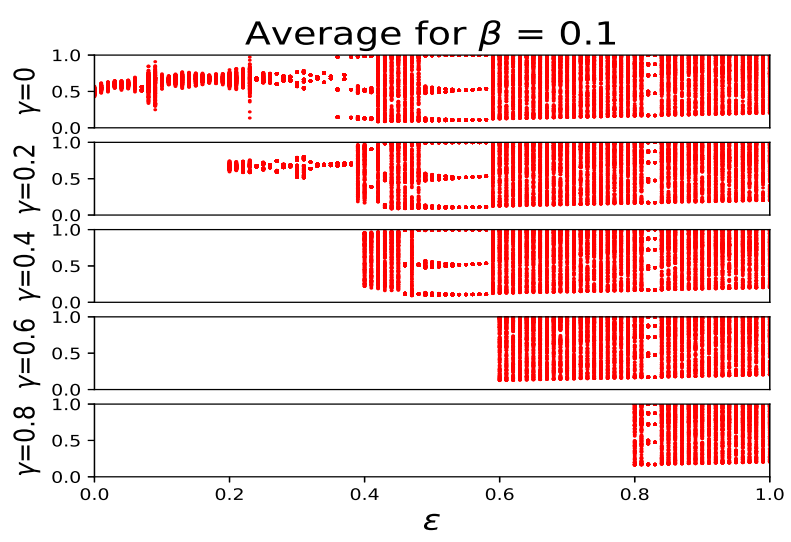

Figure 4.21: The top plot shows the result without advection. The advection is increased downwards. This shows that the type of orbits are almost the same as the DPD case. The advection have a little $(\epsilon=0.4)$ or no effect $(\epsilon>0.6)$ in presence of delay. In this case, $\alpha=0$. 
In Figure 4.21, 200 values of $h$ after the transient time $t_{0}$ are shown for each $\epsilon$. The advection has a moderate effect over the orbits and primarily reduces the interval in $\epsilon$.

For instance, the orbits around $\epsilon=0.4$, when $\gamma=0$, have a definite period (top panel). When $\gamma$ is increased, for example $\gamma=0.4$, they have a much larger period (mid panel). This is due to the effect of advection over individual trajectories of states with NLE. The advection destroys regular orbits. For orbits where the coupling is strong enough, $\epsilon \geq 0.6$, there is little or no change with advection.

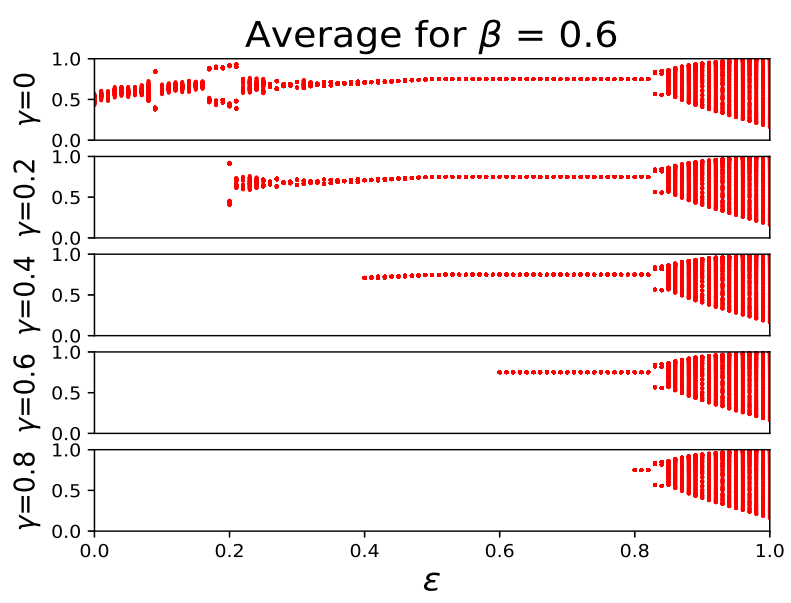

Figure 4.22: Effect of $\gamma$ on the collective metrics. In this case $\alpha=0$.

For $\beta>0.25$, due to the emergence of the stable interval for the fixed point $x^{*}$, the advection does not change the orbits in this region and only reduces the domain. This is illustrated in Figure 4.22.

From the observed isolated effect of advection and delay, and the results of the DAD model, we claim that, in general, the effects of delay overcome those of advection. It is the restriction $\gamma \leq \epsilon$ that mainly modifies the results of the DAD model when compared with the DPD model.

This can be explained by the characteristics of the advection compared with delay. We saw that the effect is weak and progressive. Delay, on the other hand, changes the CSR, the type of orbits and the states with NLE. Moreover, it allows the emergence of CS states even in the short-range case $(\alpha \rightarrow \infty)$. Also important is to recall that the advective term is asymmetric, therefore when the CS state is reached in a fixed point, that term becomes equal to zero. Orbits and collective metrics means that they are unaffected by $\gamma$. This is not the case when delay is included in the scheme, as we saw in section 4.5. More examples can be found in the Appendix E. 


\section{5}

\section{Conclusions and Perspectives}

\section{1}

\section{Conclusions}

We first revisited CMLs with diffusive coupling (SPD and DPD) models that have been studied in the literature before $[20,21,6,7,26]$. Then, we approached new cases, that as far as we know, have not been studied before, namely the CMLS with inclusion of advection (SAD and DAD) modulated by parameter $\gamma$. The SAD model is studied in [16-19] but from a different perspective and only for first neighbors range of interactions.

As a control, we verified that in the limit $\gamma \rightarrow 0$, the results previously known for the models without advection are recovered.

We focused on complete synchronization (CS). In the synchronous cases (without delay, that is $\beta=0$ ), CS occurs in chaotic trajectories. We delimited analytically the region in parameter space where CS occurs (CSR), by means of the study of transversal stability. Numerical simulation and verifications were also performed.

In the cases with delay (that is, $\beta>0$ ), CS occurs in regular trajectories. In this case, we obtained analytically the frontier of domains only for the case of period 1 orbits. Moreover, delays facilitate CS that can occur even for short-range interactions.

In all cases advection is prejudicial for CS stability. Furthermore, we also analyzed the effects of advection on regular trajectories (even if the collective state is not synchronized).

We wish to emphasize three evinced phenomena. The first, is that advection destroys regular orbits $(\lambda<0)$ and completely synchronized states $(\sigma=0)$. This is done by spreading out individual trajectories as shown in Chapter 4. This separation makes $\sigma \neq 0$ and $\lambda$ less negative but not necessarily positive. 


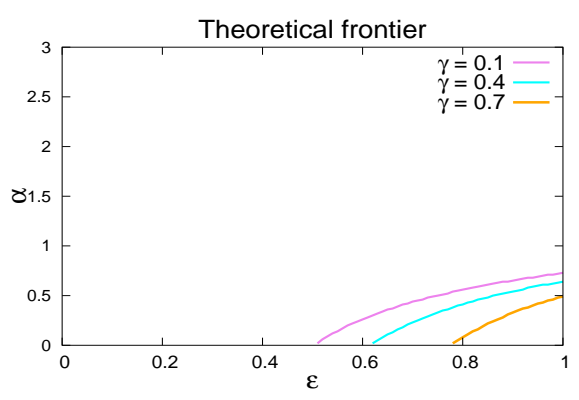

5.1(a): Advection on CS states.

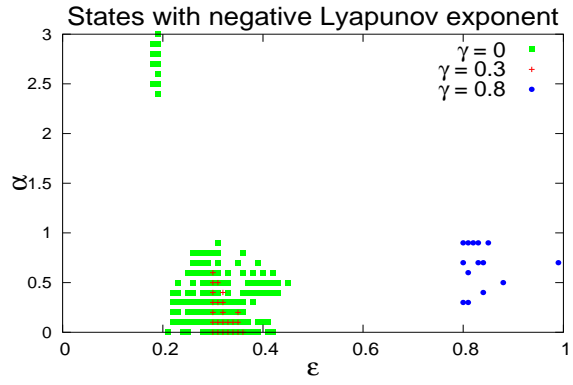

5.1(b): Advection on NLE states.

Figure 5.1: Isolated effect of advection in the SAD model.

The second idea is that the delay favors CS and NLE. This is not monotonic in $\beta$. For instance, this is due to the stability region of the CS states around the fixed point.

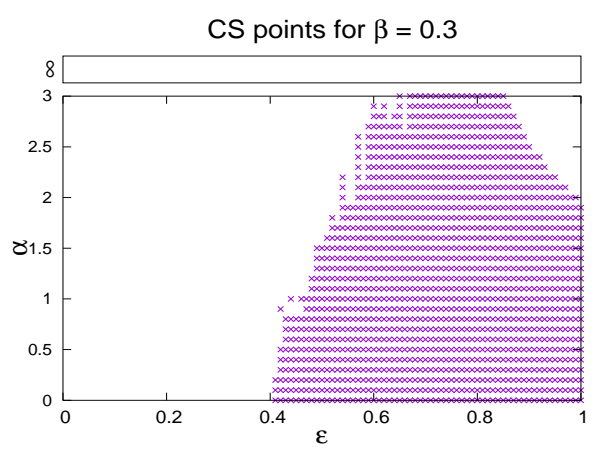

5.2(a): CSR states with $\beta=0.3$

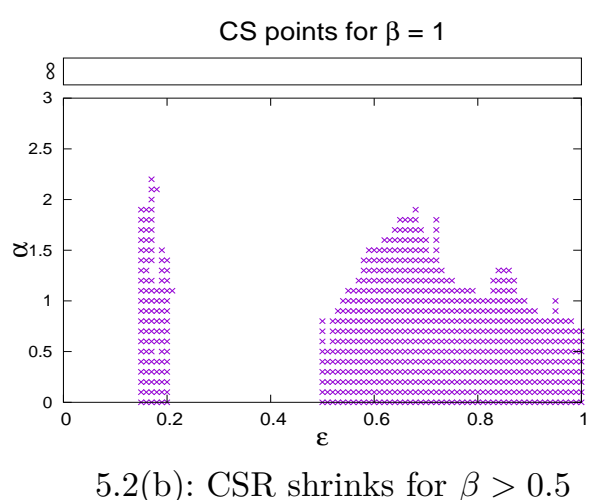

5.2(b): CSR shrinks for $\beta>0.5$

Figure 5.2: The effect of delay on CS states.

Finally, when advection and delay are together in the DAD model, delay dominates the dynamics, hindering the effects of advection described above.

\section{2}

\section{Perspectives}

An immediate perspective of the present work is to extend the studies done for the cases with delay to CS orbits other than period 1 trajectories.

Another perspective is to identify and classify phase synchronization. In this line a new collective metric was initially developed to measure the Complete Phase Synchronization, CPS.

The idea is to look for harmonious collective coherence without considering phase shifts. We have already done preliminary work in this direction. The new metric is based on [29-32]. In [31, 32], for two-dimensional CMLs, the 
authors define a new variable as the difference between two consecutive states of the system. In [29], also for a two-dimensional CML, the authors define an instantaneous order parameter as the average of the sign of each map. This last idea is applied in [30] for a one-dimensional CML.

We consider an individual map and the difference between its current state, $x_{t+1}^{i}$, and the previous one, $x_{t}^{i}$. This can be defined as $z$.

$$
z_{t+1}^{i}=x_{t+1}^{i}-x_{t}^{i} .
$$

Positive values are associated with increasing slope and negative ones with decreasing slope. The problem with $z$ is the scale. Two maps can have different values of $z$, even if they are moving in-phase. To overcome this issue we use the sign function and define a new variable $w$.

$$
w_{t+1}^{i}=\operatorname{sgn}\left(z_{t+1}^{i}\right) .
$$

This gives us a +1 every time that an individual map moves up and a -1 when it moves down. Then, if two maps, $k$ and $j$, are in-phase, meaning that they move at the same time up or down, $w_{t}^{k}=w_{t}^{j}$. If so, regardless the actual value of $z_{t}^{k}$ and $z_{t}^{j}$, the multiplication of the components of $w$ will be 1 . So, we can calculate this metric for each map, for each time step, taking as reference any individual trajectory, that is

$$
\phi_{t}^{i}=w_{t}^{i} \times w_{t}^{1} .
$$

Finally we can take the spatial average to look at the evolution of this metric in the parameter space, obtaining the collective quantity

$$
\phi_{t}=\frac{1}{N-1} \sum_{i=1}^{N-1} \phi_{t} z^{i}
$$

Like with the other metrics, we take the temporal average over the last 200 iterations. It is important to notice that $\phi_{t}$ differs from those described in [5], because they are conceived for master-slave systems, where the phase synchronization is measured against the master system.

We tested $\phi_{t}$ with two models: SPD and SAD.

In terms of the complete phase synchronization metric, CS states have $\phi$ equal to 1 . When the coupling is low $\epsilon \approx 0$, the system is a collection of uncoupled chaotic logistic maps that should not have a preferred direction at any time. Furthermore, we could expect that at any step, roughly, half of the systems will go up and the other half down, therefore we should get an average value of $\phi \approx 0$. The states with CPS match with the CS in this model. 


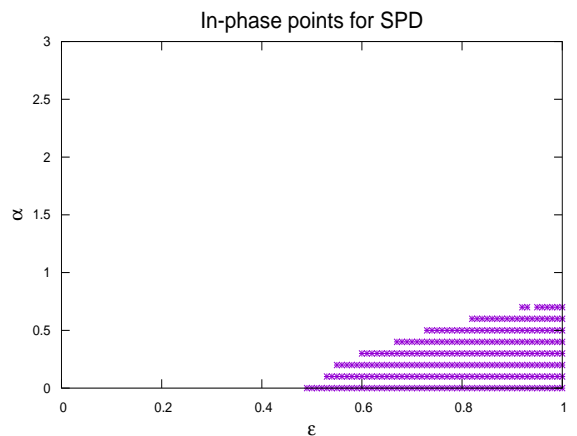

5.3(a): Points where $\phi=1$

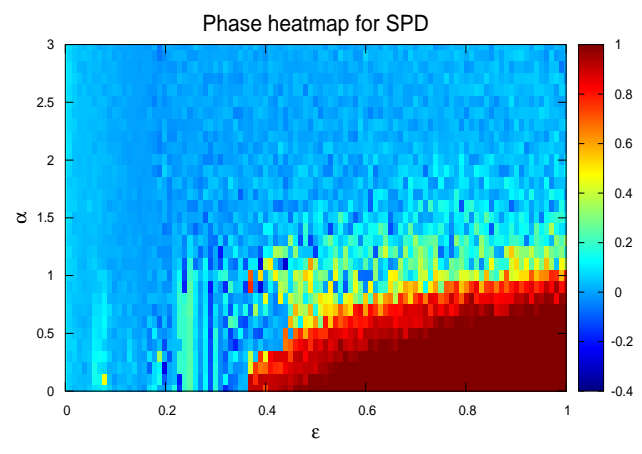

5.3(b): Heatmap for $\phi$

Figure 5.3: CPS appears in the CS zone.

In the purely diffusive model, the states with $\phi=1$ coincide with the CSR. In the model with advection, they do not necessarily are the same, and depend upon the value of the advection parameter. For example, for $\gamma=0.5$ they do not match.

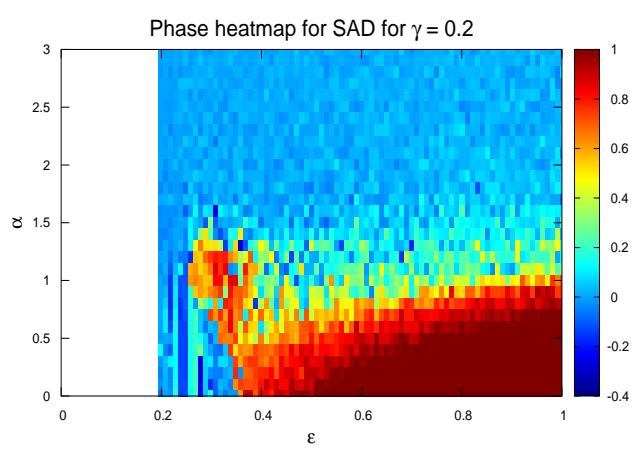

5.4(a): Heatmap of $\phi, \gamma=0.2$

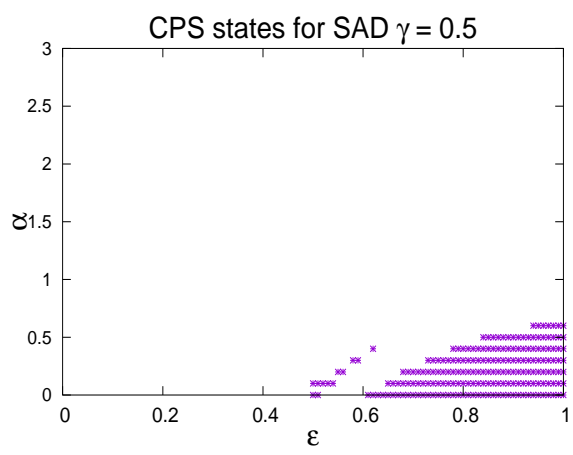

$5.4(\mathrm{~b}): \phi=1, \gamma=0.5$

Figure 5.4: $\phi$ becomes homogeneous with increasing advection.

For this model, $\phi_{t}$ turns out to be quite homogeneous and for that reason, the heatmap is not very useful. Nevertheless, in the same way that the deviation identifies states with $\sigma=0$, this metric can be specialized for $\phi=1$. 
A

\section{Appendix A: Numerical results for the SPD model}

Individual trajectories, for 12 maps from $N=201$, are shown in Figures A.1 and A.2 in order to illustrate the types of orbits mentioned in Figure 4.2(b). The maps in each case are the same: $[1,30,40,60,70,90,100,150,170,180,190,201]$.

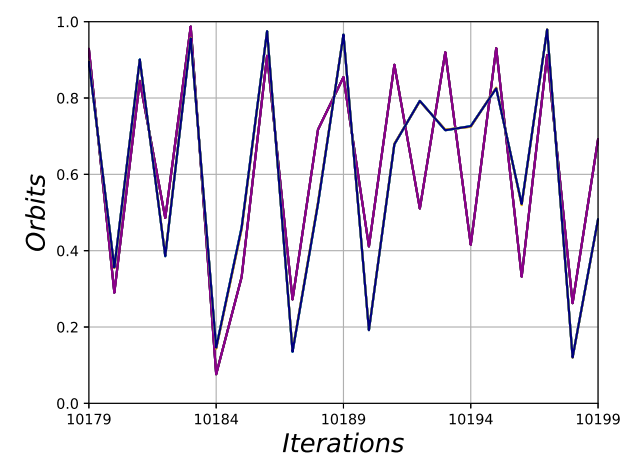

A.1(a): $(\alpha, \epsilon)=(0.0,0.4)$

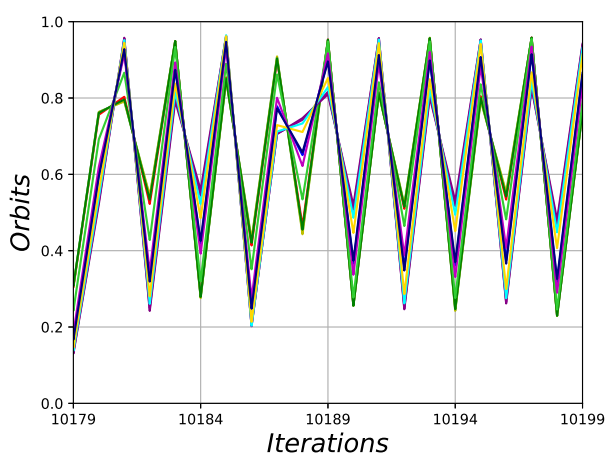

A.1(b): $(\alpha, \epsilon)=(0.5,0.6)$

Figure A.1: The green zone of Figure 4.2(b) has non periodic clusters and quasi synchronized states.

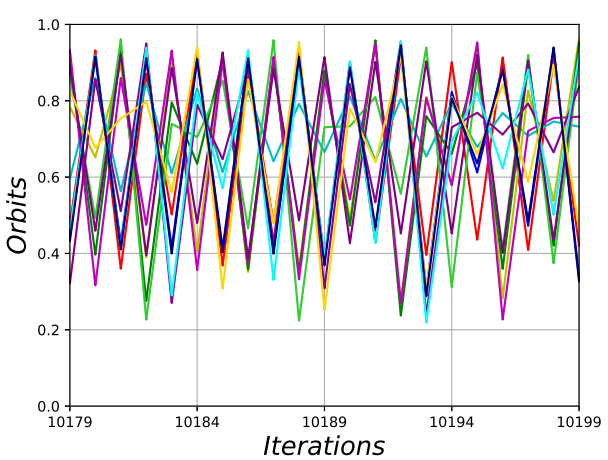

A.2(a): Yellow zone $(\alpha, \epsilon)=(1.5,0.8)$

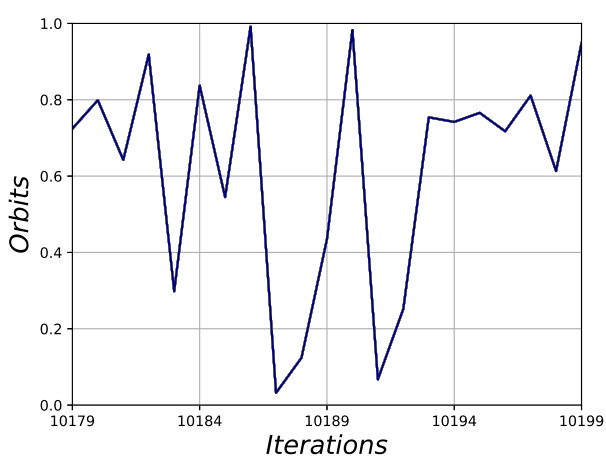

A.2(b): White zone $(\alpha, \epsilon)=(0.3,0.8)$

Figure A.2: Yellow and white zones of Figure 4.2(b).

Examples for the red zone are omitted because trajectories are very similar to a set of uncoupled maps. 
B

\section{Appendix B: Numerical results for the SAD model}
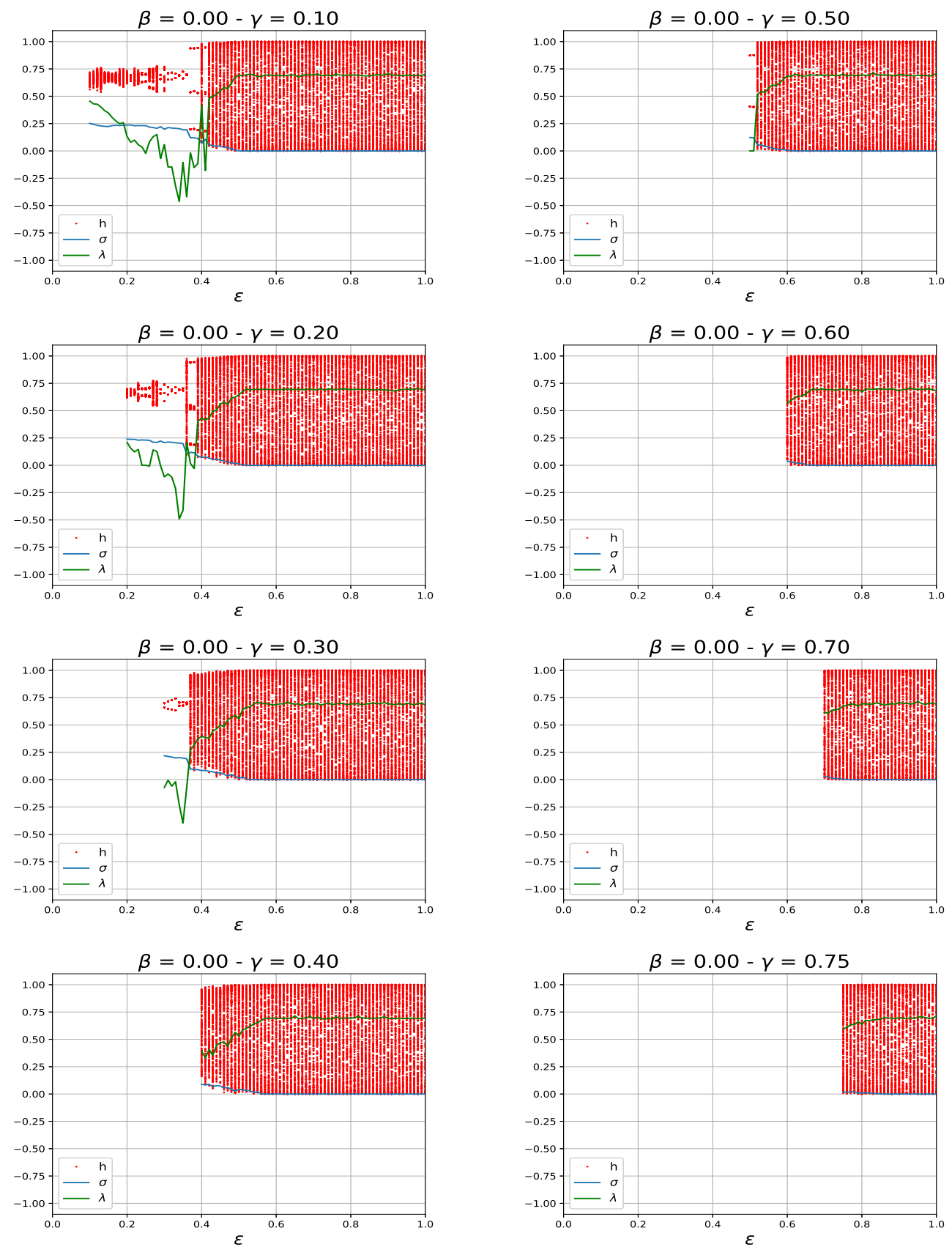

Figure B.1: Collective metrics $h, \sigma$ and $\lambda$ for the SAD model at $\alpha=0$. Advection causes the reduction of the synchronization domain. Each panel correspond to a different value of $\gamma$. 

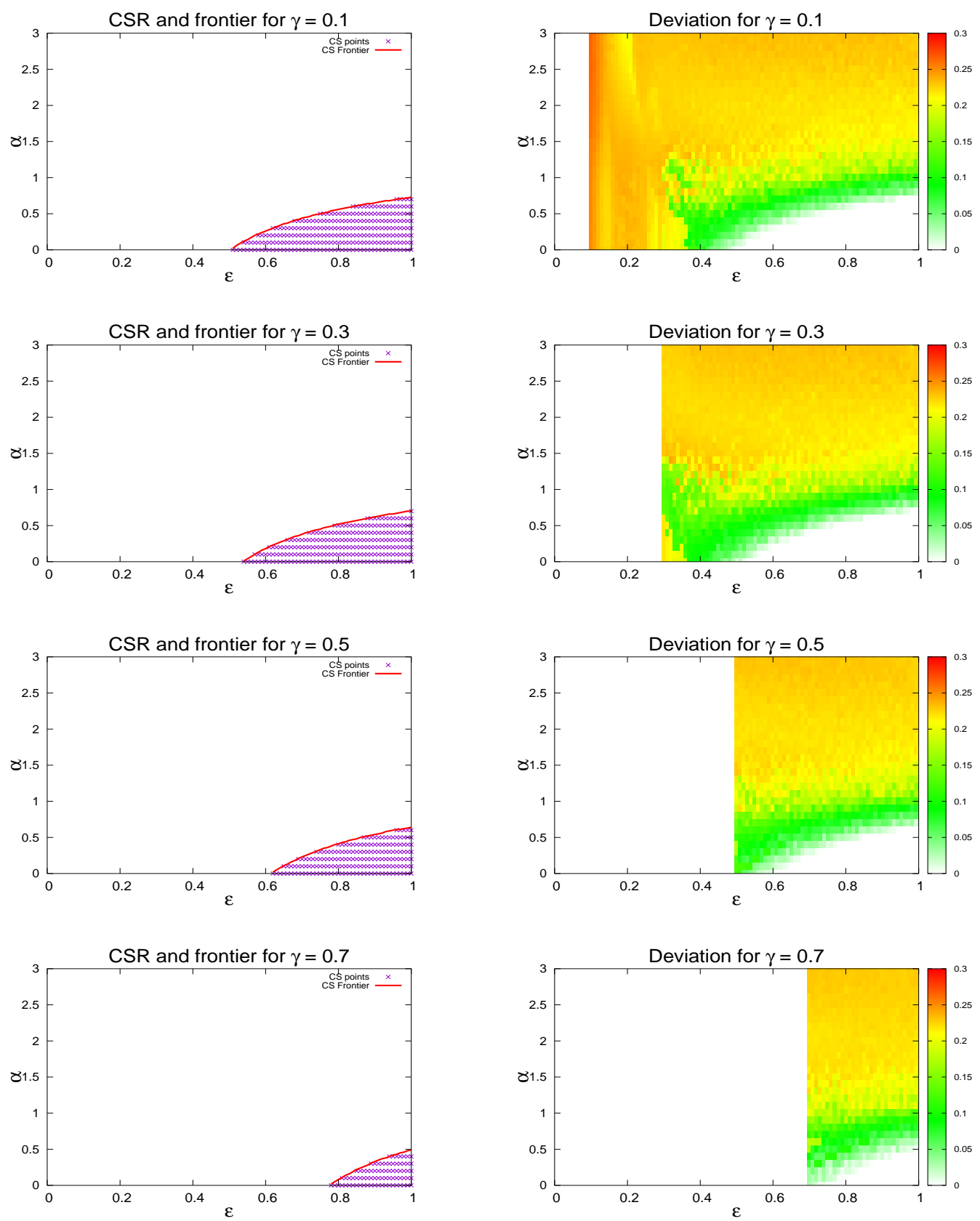

Figure B.2: In the left column the theoretical frontier and the complete synchronization region (CSR) from simulations. The effect of advection, from $\gamma=0.1$ to 0.7 is to reduce the CSR. In the right column the average value for $\sigma$ in the parameter space is shown. 

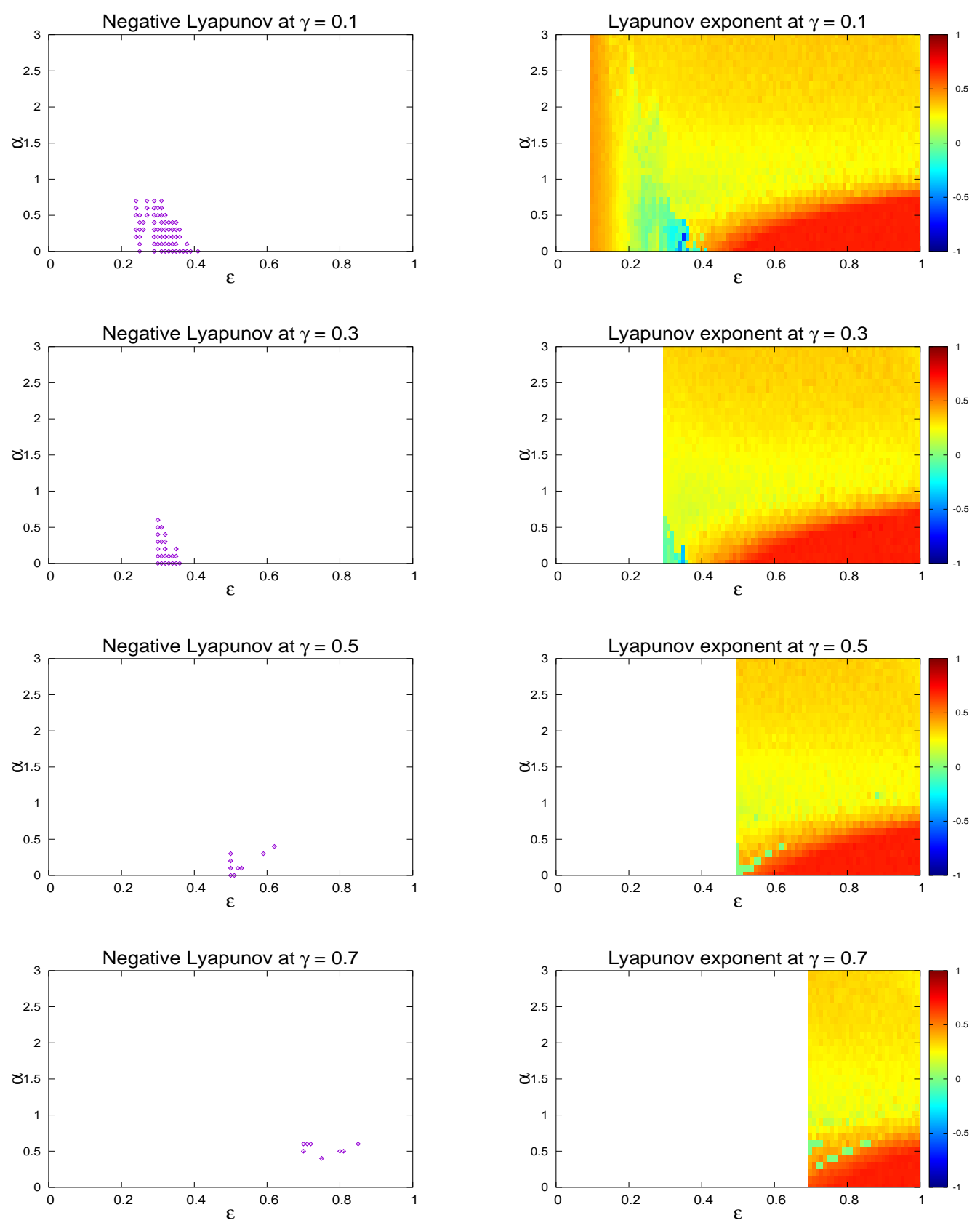

Figure B.3: Lyapunov exponent values for the SAD model. In the left column, the states with negative Lyapunov exponent (NLE) which are modified by the effect of advection: advection destruct this type of states. In the right column, the average value for $\lambda$ in the parameter space is shown. 
C

\section{Appendix C: Numerical results for the DPD model}
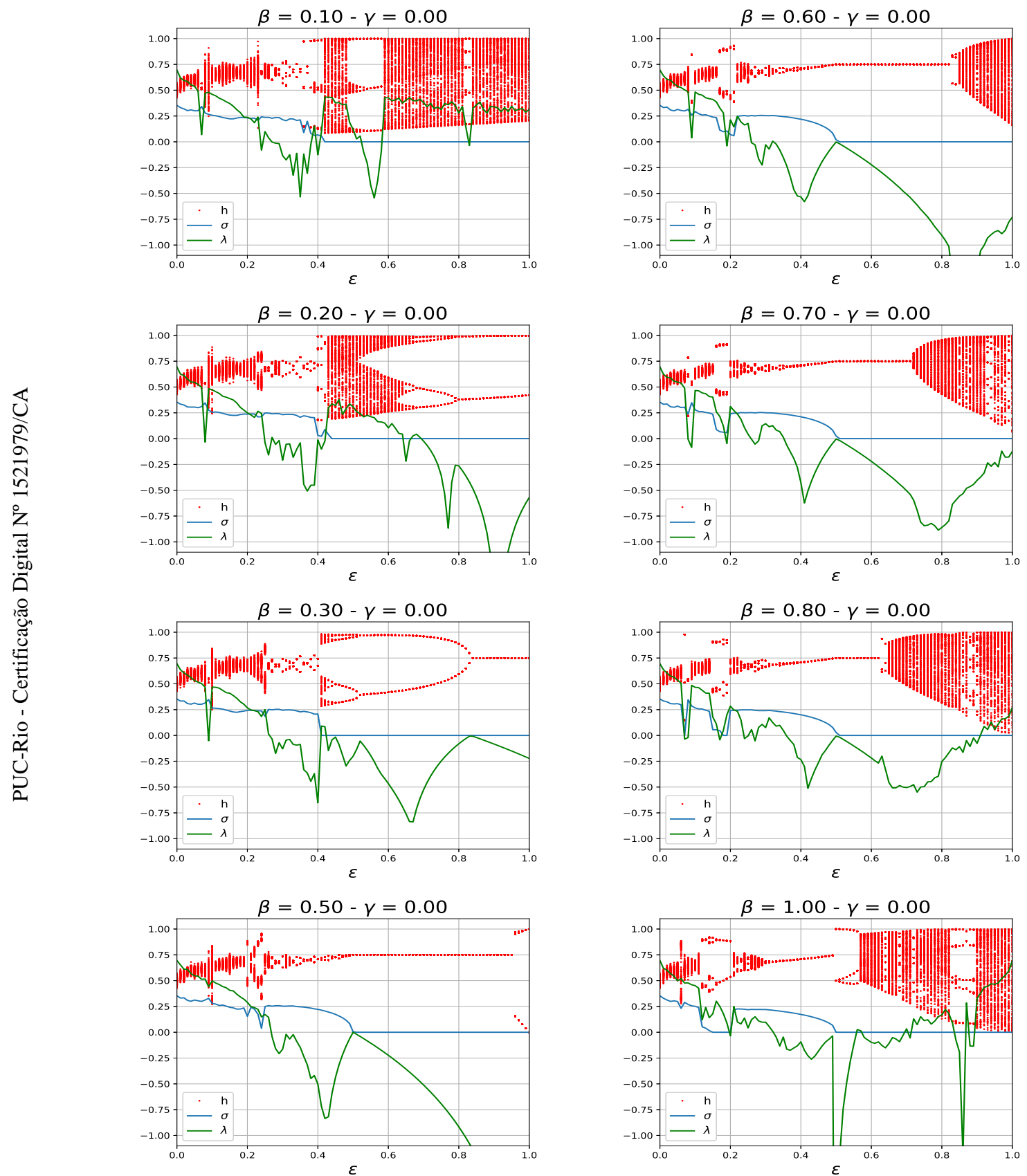

Figure C.1: Collective metrics $h, \sigma$ and $\lambda$ in the DPD model for $\alpha=0$. The delay favors synchronization. Large enough $\beta$ makes chaotic orbits emerge, as shown in the last panel in the right column. 

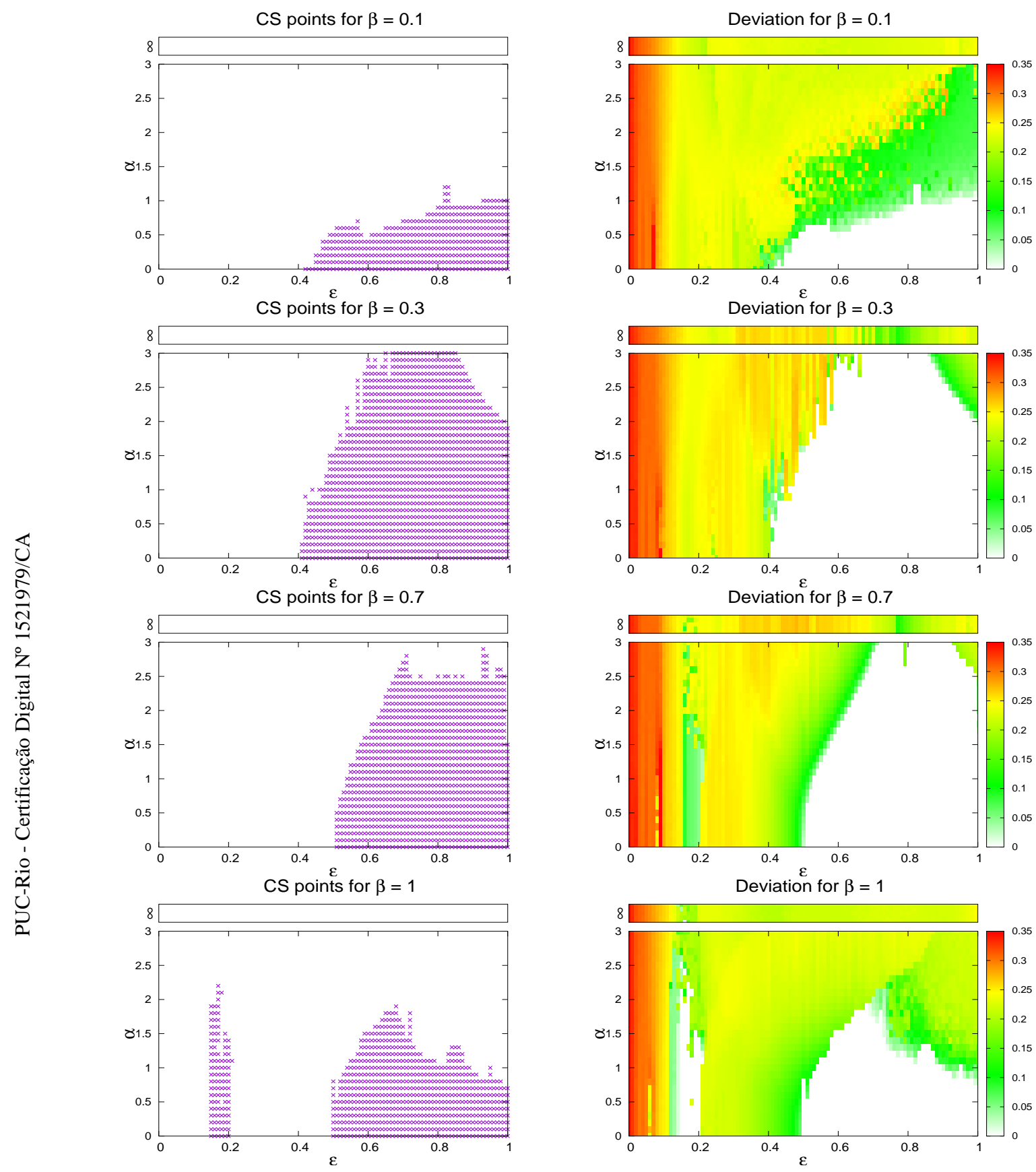

Figure C.2: In the left column, CS states for different values of $\beta$ are shown. In all cases, the delay favors the synchronization. In the right column, the average value of $\sigma$ for different values of $\beta$ is shown in the parameter space. 

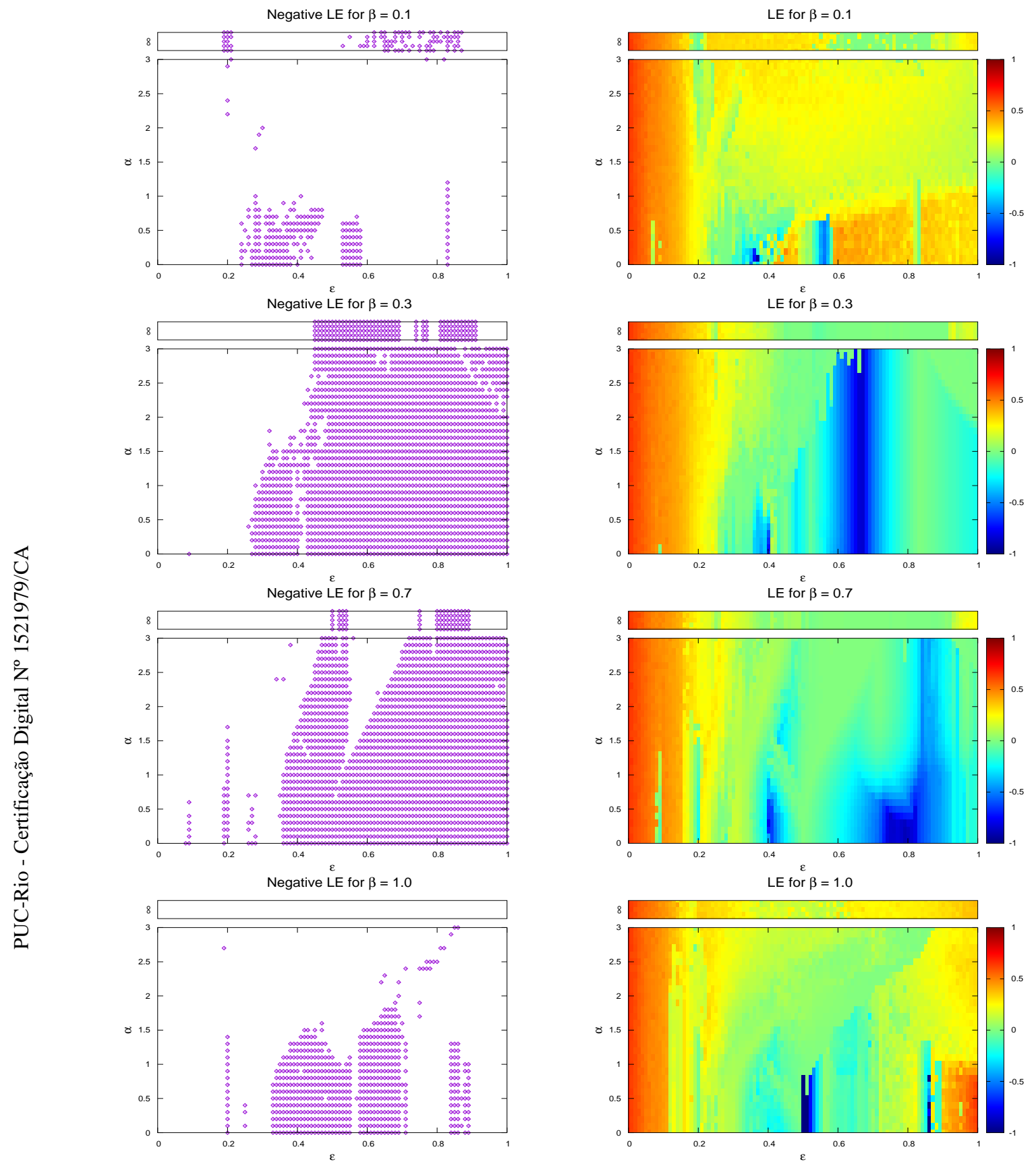

Figure C.3: Lyapunov exponent values for the DPD model. In the left column, states with negative Lyapunov exponent are modified by the effect of delay. In the right column the average value for $\lambda$ is shown for different values of $\beta$, in the parameter space. 
D

\section{Appendix D: Numerical results for the DAD model}
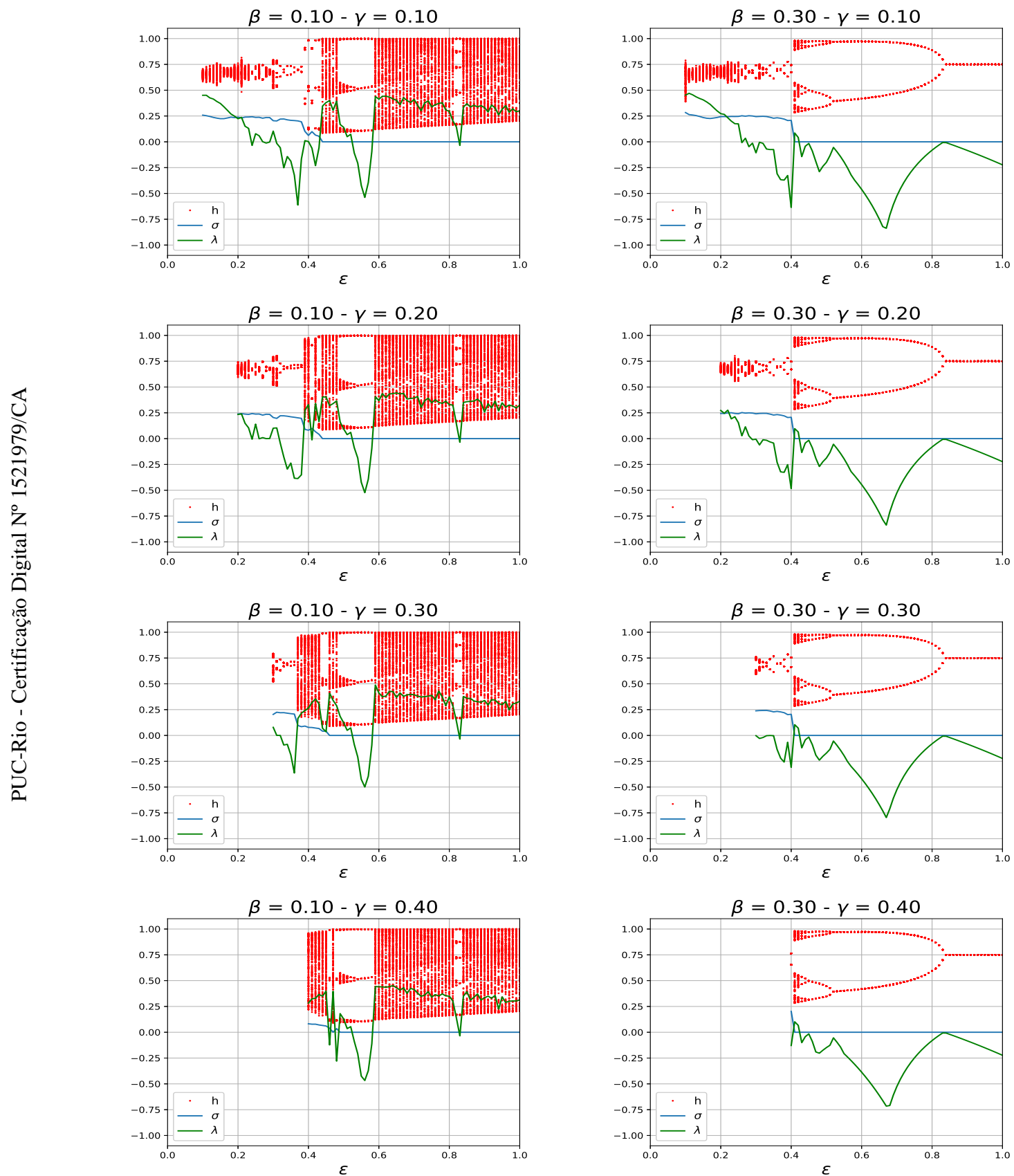

Figure D.1: Collective metrics $h, \sigma$ and $\lambda$ in the DAD model for $\alpha=0$. In the left column for $\beta=0.1, \gamma$ goes from 0.1 (upper panel) to 0.4 (lower panel). In the right column for $\beta=0.3, \gamma$ goes from 0.1 (upper panel) to 0.4 (lower panel). The advection reduces the CS domain. 

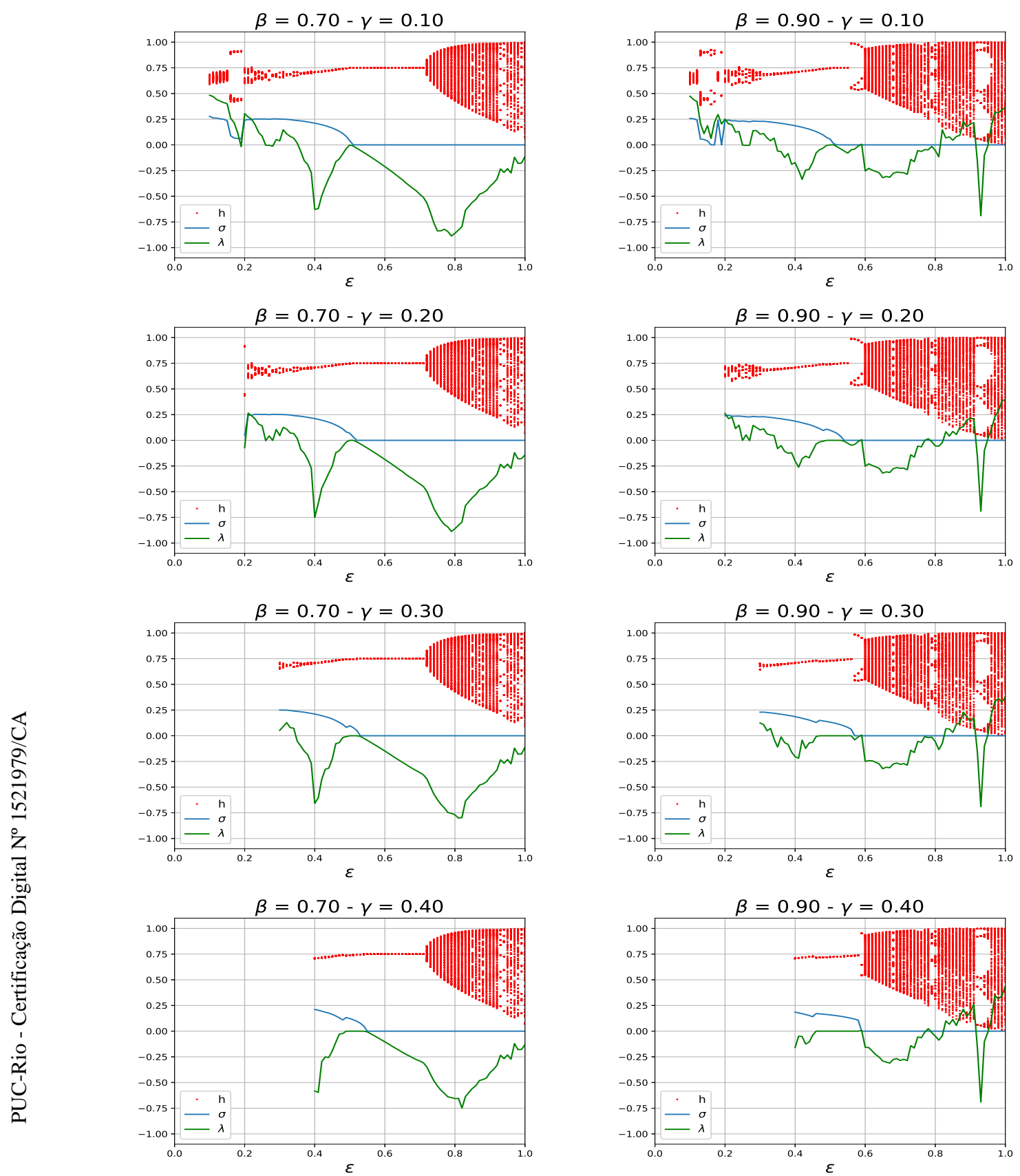

Figure D.2: Collective metrics $h, \sigma$ and $\lambda$ in the DAD model for $\alpha=0$. In the left column for $\beta=0.7, \gamma$ goes from 0.1 (upper panel) to 0.4 (lower panel). In the right column for $\beta=0.9, \gamma$ goes from 0.1 (upper panel) to 0.4 (lower panel). The advection reduces the CS domain. 

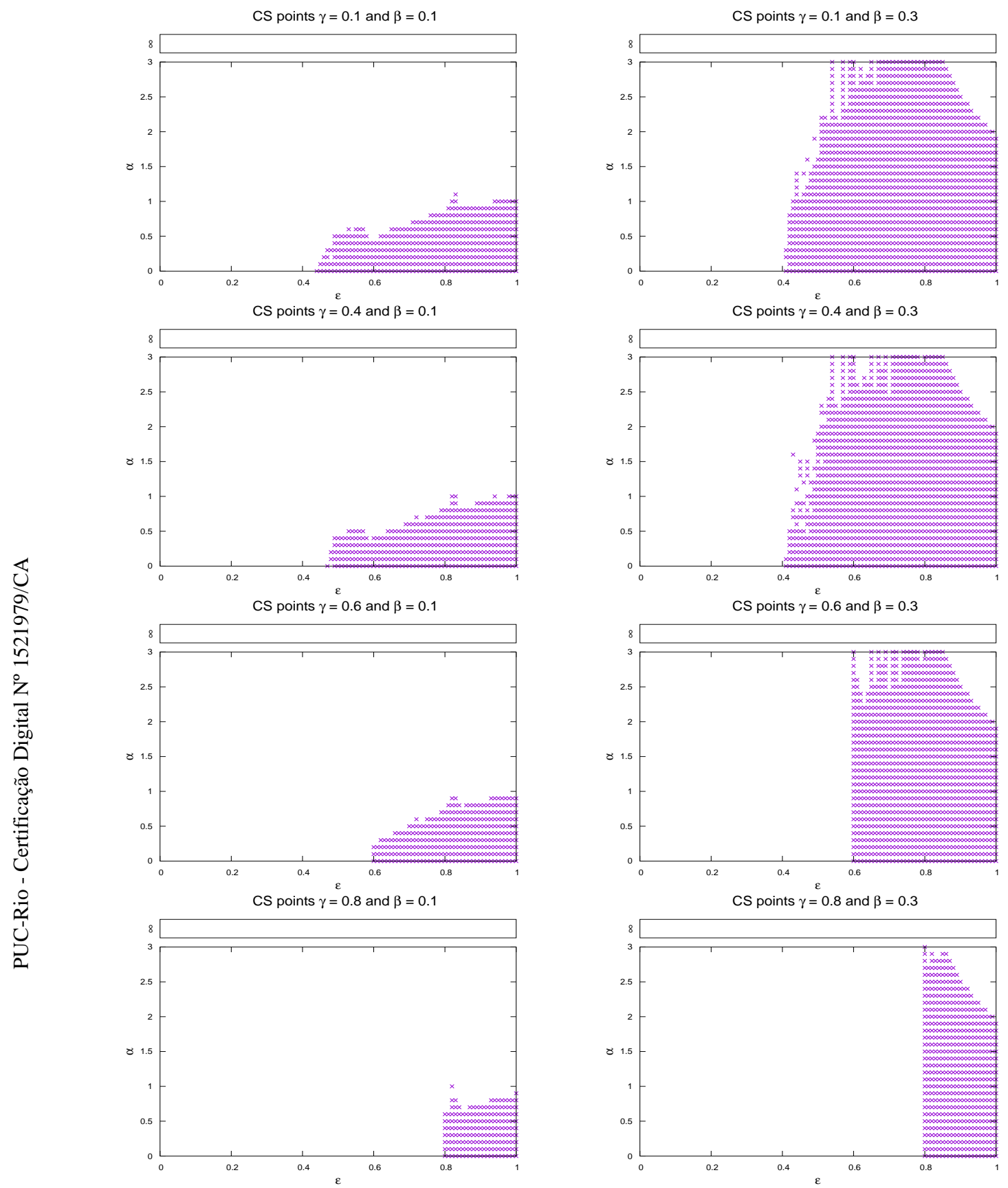

Figure D.3: CS states in the DAD model. In the left column for $\beta=0.1, \gamma$ goes from 0.1 (upper panel) to 0.8 (lower panel). In the right column for $\beta=0.3$, $\gamma$ goes from 0.1 (upper panel) to 0.8 (lower panel). The advection reduces the CS domain while the delay favor CS states. 

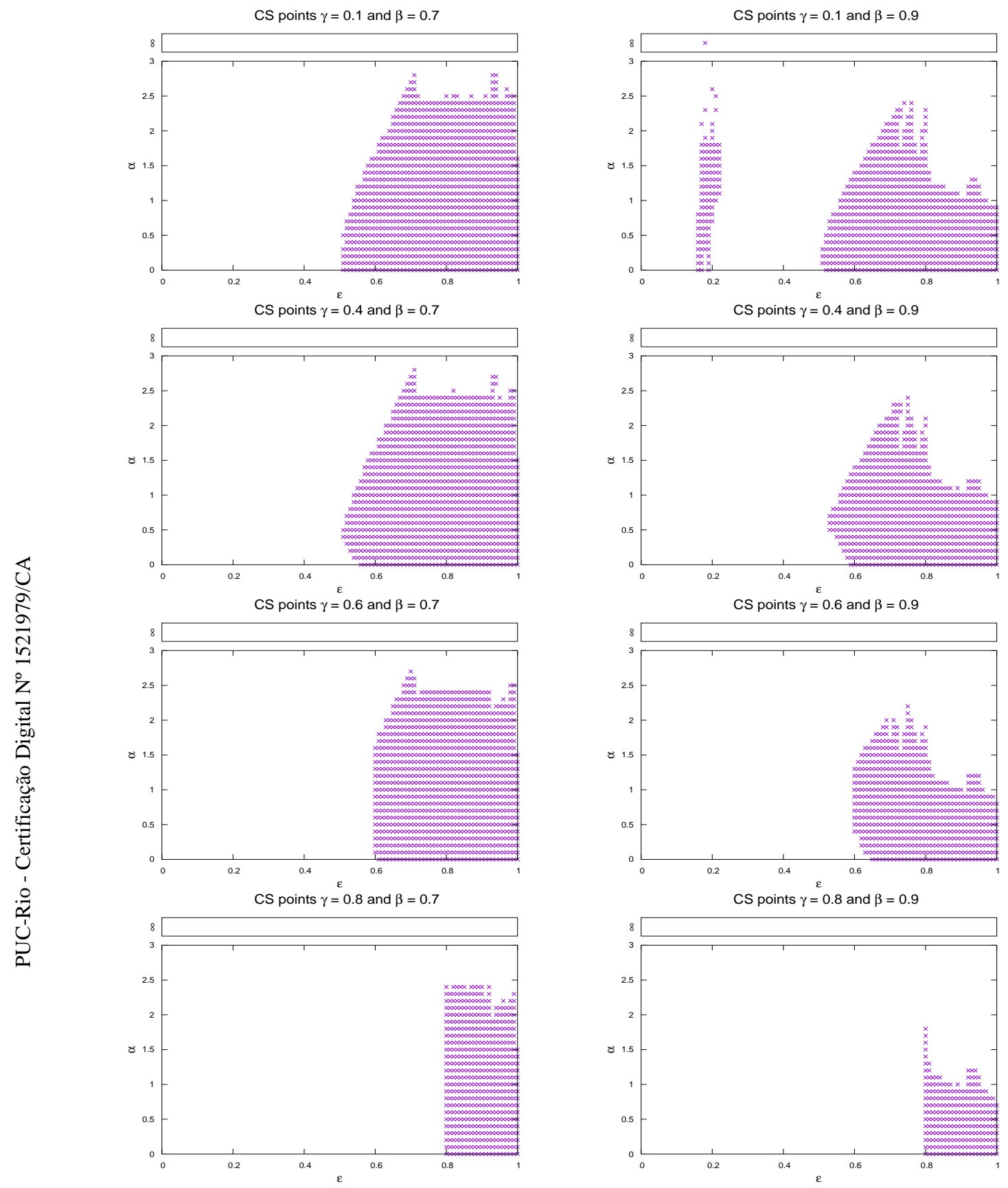

Figure D.4: CS states in the DAD model. In the left column for $\beta=0.7, \gamma$ goes from 0.1 (upper panel) to 0.8 (lower panel). In the right column for $\beta=0.9$, $\gamma$ goes from 0.1 (upper panel) to 0.8 (lower panel). The advection reduces the CS domain while the delay favor CS states. 

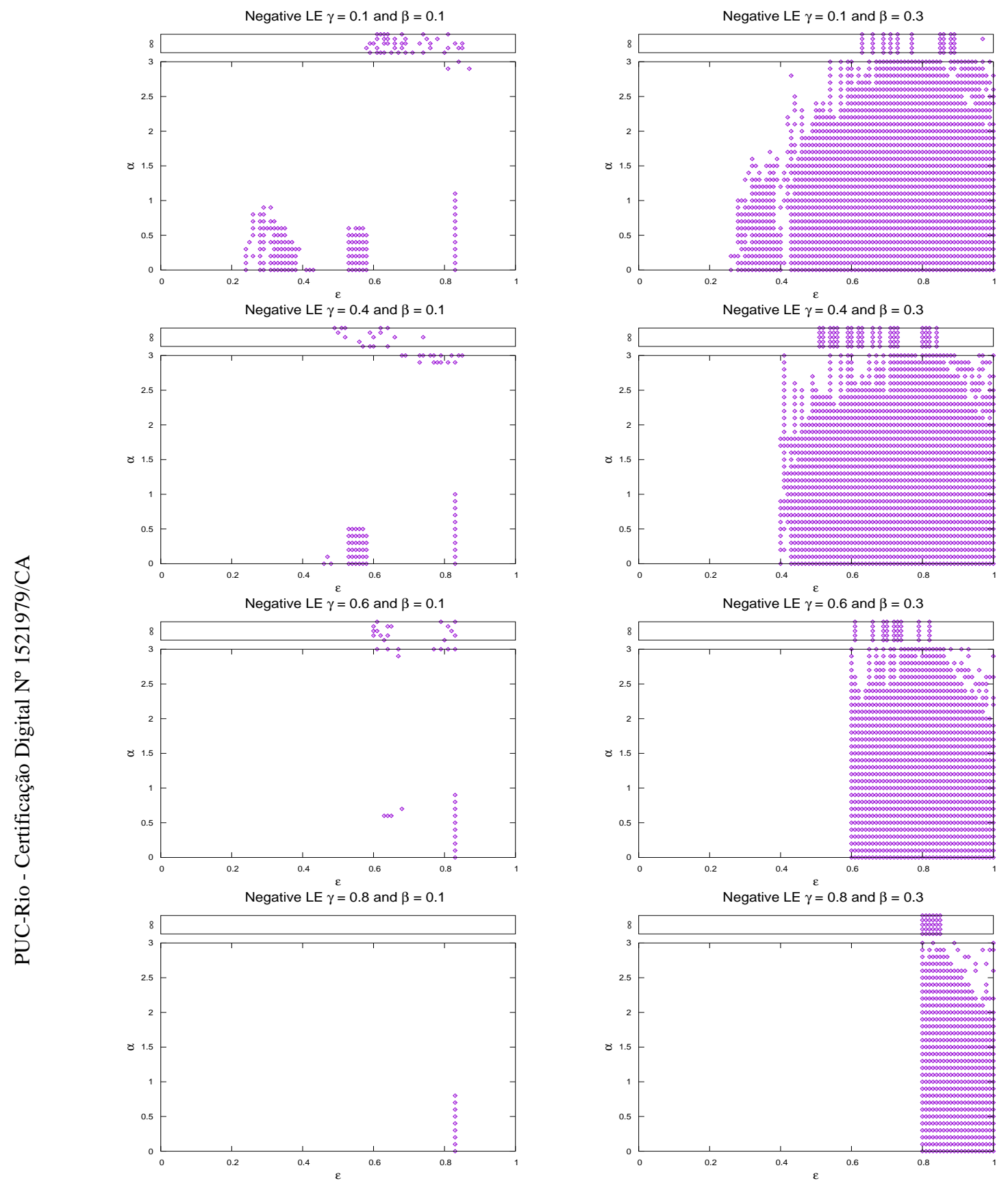

Figure D.5: Negative Lyapunov exponent in the DAD model. In the left column for $\beta=0.1, \gamma$ goes from 0.1 (upper panel) to 0.8 (lower panel). In the right column for $\beta=0.3, \gamma$ goes from 0.1 (upper panel) to 0.8 (lower panel). The advection reduces the number of this type of states. 

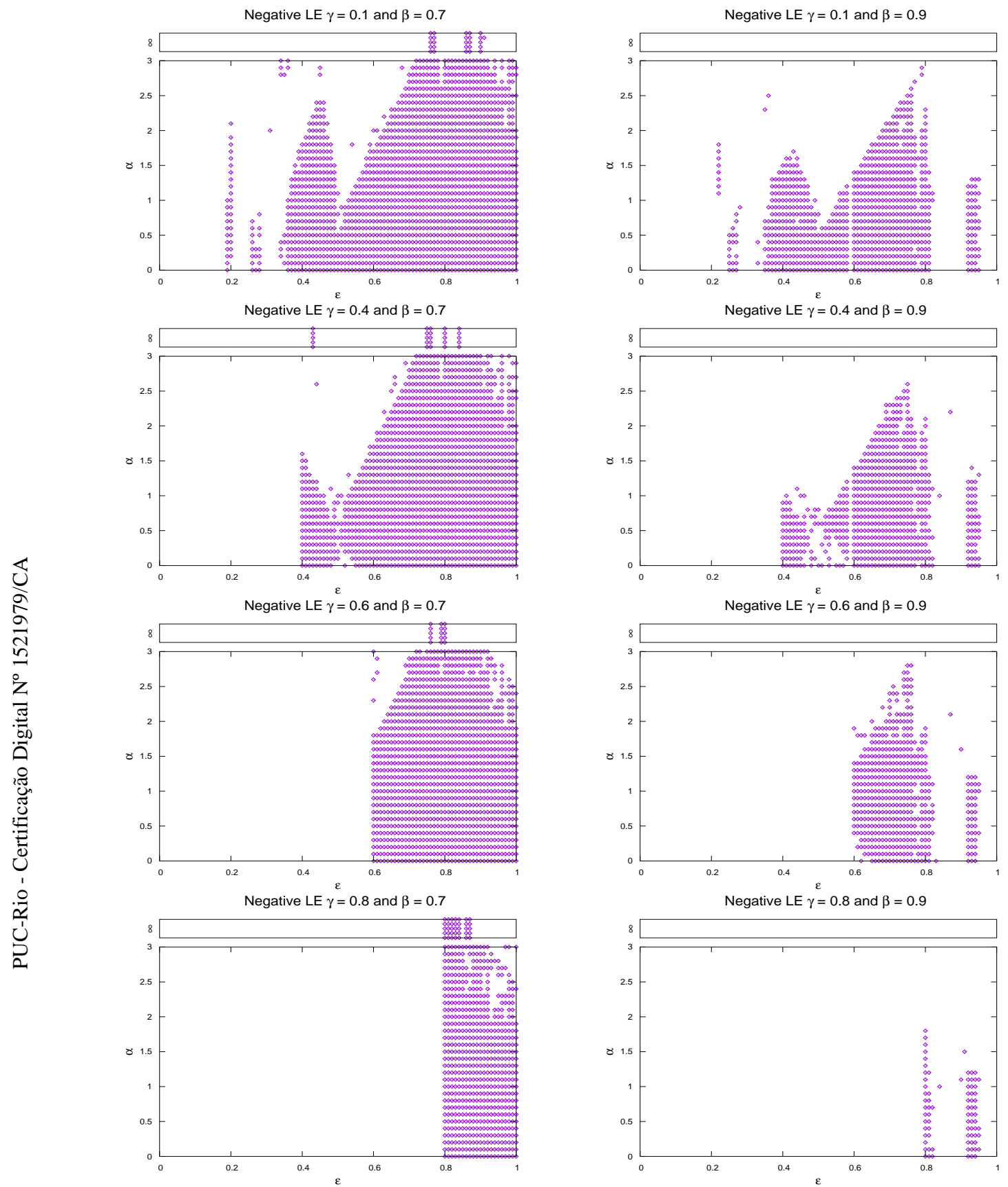

Figure D.6: Negative Lyapunov exponent in the DAD model. In the left column for $\beta=0.7, \gamma$ goes from 0.1 (upper panel) to 0.8 (lower panel). In the right column for $\beta=0.9, \gamma$ goes from 0.1 (upper panel) to 0.8 (lower panel). The advection reduces the number of this type of states. 
E

\section{Appendix E: Other numerical results}
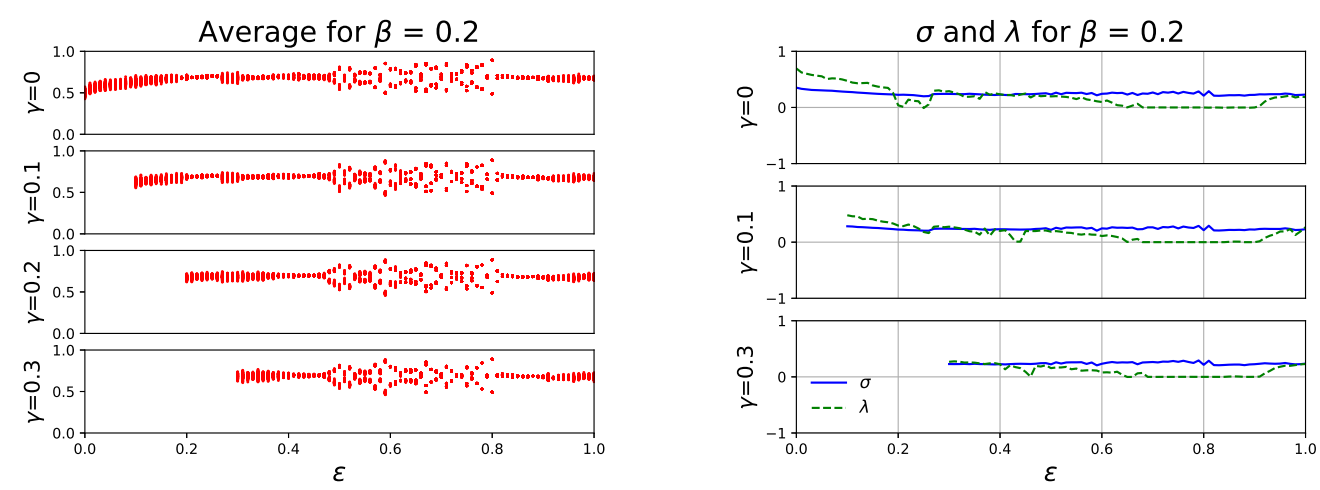

Figure E.1: For $\beta=0.2$, in the DAD model, the effect of $\gamma$ in the collective metrics is shown. When $\alpha=50$, the advection only reduce the domain in $\epsilon$.
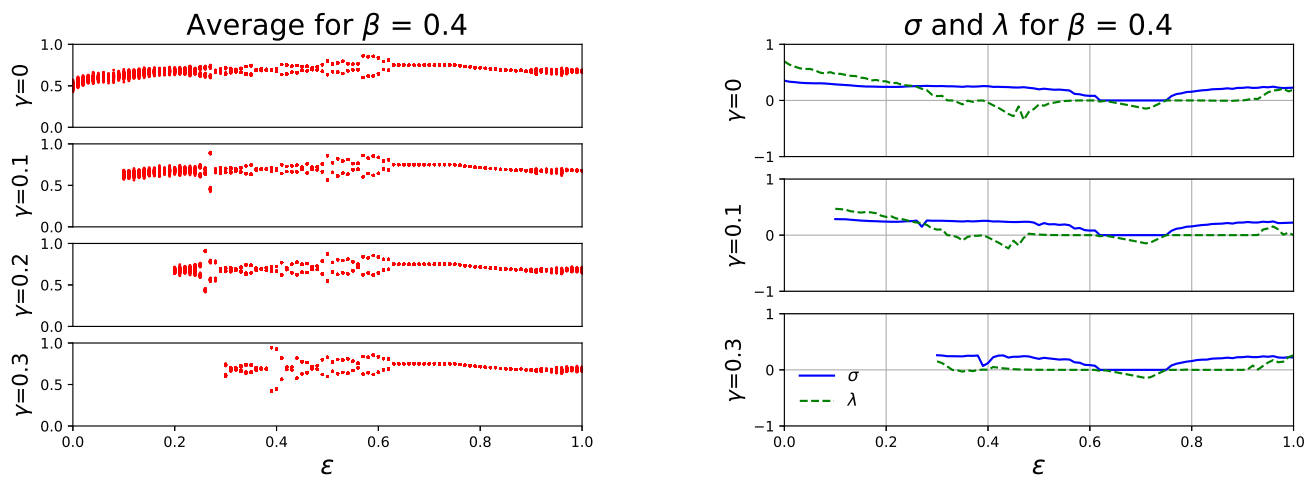

Figure E.2: The same phenomenon described above occurs for $\beta=0.4$. This means that the effect of the range of interactions is stronger than the effect of delay and advection.

The effect of advection for $\alpha \rightarrow \infty$ over the complete synchronized states and those with negative Lyapunov exponent (NLE) is the similar. In Figure E.1 there is no effect; in those states we do not have CS nor NLE. In Figure E.2, over NLE states, makes their $\lambda$ less negative. There is no effect over CS states because they belong to the stability region of the fixed point. 


\section{Bibliography}

[1] PIKOVSKY, A.. Synchronization : a universal concept in nonlinear sciences. Cambridge University Press, Cambridge, 2001.

[2] VLASOV, V.; PIKOVSKY, A.. Synchronization of a josephson junction array in terms of global variables. Phys. Rev. E, 88:022908, Aug 2013.

[3] GLASS, L.. Synchronization and rhythmic processes in physiology. Nature, 410(6825):277-284, mar 2001.

[4] PÉREZ, A.; CARREIRAS, M. ; DUÑABEITIA, J. A.. Brain-to-brain entrainment: EEG interbrain synchronization while speaking and listening. Scientific Reports, 7(1), jun 2017.

[5] BOCCALETTI, S.; KURTHS, J.; OSIPOV, G.; VALLADARES, D. ; ZHOU, C.. The synchronization of chaotic systems. Physics Reports, 366(1):1 $-101,2002$.

[6] ANTENEODO, C.; BATISTA, A. ; VIANA, R.. Chaos synchronization in long-range coupled map lattices. Physics Letters A, 326(3):227 233, 2004.

[7] ANTENEODO, C.; BATISTA, A. ; VIANA, R.. Synchronization threshold in coupled logistic map lattices. Physica D: Nonlinear Phenomena, 223(2):270-275, nov 2006.

[8] KANEKO, K.. Overview of coupled map lattices. Chaos: An Interdisciplinary Journal of Nonlinear Science, 2(3):279-282, jul 1992.

[9] KANEKO, K.. Pattern dynamics in spatiotemporal chaos. Physica D: Nonlinear Phenomena, 34(1-2):1-41, jan 1989.

[10] KANEKO, K.. Clustering, coding, switching, hierarchical ordering, and control in a network of chaotic elements. Physica D: Nonlinear Phenomena, 41(2):137-172, mar 1990.

[11] GUPTE, N.; SHARMA, A. ; PRADHAN, G. R.. Dynamical and statistical behaviour of coupled map lattices. Physica A: Statistical Mechanics and its Applications, 318(1-2):85-91, feb 2003. 
[12] BUNIMOVICH, L. A.; LAMBERT, A. ; LIMA, R.. The emergence of coherent structures in coupled map lattices. Journal of Statistical Physics, 61(1-2):253-262, oct 1990.

[13] JUST, W.. Bifurcations in globally coupled map lattices. Journal of Statistical Physics, 79(1-2):429-449, apr 1995.

[14] VASCONCELOS, D.; VIANA, R.; LOPES, S.; BATISTA, A. ; DE S. PINTO, S.. Spatial correlations and synchronization in coupled map lattices with long-range interactions. Physica A: Statistical Mechanics and its Applications, 343(Supplement C):201 - 218, 2004.

[15] DING, M.; YANG, W.. Stability of synchronous chaos and on-off intermittency in coupled map lattices. Physical Review E, 56(4):40094016, oct 1997.

[16] LIND, P. G.; CORTE-REAL, J. A. M. ; GALLAS, J. A. C.. The distribution of periodic and aperiodic pattern evolutions in rings of diffusively coupled maps. International Journal of Bifurcation and Chaos, 11(10):2647-2661, oct 2001.

[17] LIND, P. G.; CORTE-REAL, J. ; GALLAS, J. A. C.. Modeling velocity in gradient flows with coupled-map lattices with advection. Physical Review E, 66(1), jul 2002.

[18] LIND, P. G.; CORTE-REAL, J. ; GALLAS, J. A. C.. Pattern formation in diffusive-advective coupled map lattices. Physical Review E, 69(6), jun 2004.

[19] LIND, P. G.; CORTE-REAL, J. A. ; GALLAS, J. A.. Using advection to control the velocity of patterns in rings of maps. Physica $D$ : Nonlinear Phenomena, 168-169:93-105, aug 2002.

[20] GONZÁLEZ-AVELLA, J. C.; ANTENEODO, C.. Complete synchronization equivalence in asynchronous and delayed coupled maps. Phys. Rev. E, 93:052230, May 2016.

[21] ANTEneOdO, C.; GOnZÁleZ-AVELlA, J. C. ; VAlLEjos, R. O.. From synchronous to one-time delayed dynamics in coupled maps. Phys. Rev. E, 95:062213, Jun 2017.

[22] ALLIGOOD, K.. Chaos : an introduction to dynamical systems. Springer, New York, 1997. 
[23] STROGATZ, S.. Nonlinear dynamics and chaos : with applications to physics, biology, chemistry, and engineering. Westview Press, a member of the Perseus Books Group, Boulder, CO, 2015.

[24] DEVANEY, R.. A first course in chaotic dynamical systems : theory and experiment. Addison-Wesley, Reading, Mass, 1992.

[25] OTT, E.. Chaos in dynamical systems. Cambridge University Press, Cambridge, U.K. New York, 2002.

[26] ANTENEODO, C.; DE S. PINTO, S. E.; BATISTA, A. M. ; VIANA, R. L.. Analytical results for coupled-map lattices with long-range interactions. Physical Review E, 68(4), oct 2003.

[27] JAMES, F.. A review of pseudorandom number generators. Computer Physics Communications, 60(3):329 - 344, 1990.

[28] SPROTT, J.. Chaos and time-series analysis. Oxford University Press, Oxford New York, 2003.

[29] SASTRE, F.; PÉREZ, G.. Phase transitions in lattices of coupled chaotic maps and their dependence on the local lyapunov exponent. Physical Review E, 57(5):5213-5216, may 1998.

[30] ALVAREZ-LLAMOZA, O.; COSENZA, M. G.. Synchronization and Phase Ordering in Globally Coupled Chaotic Maps, p. 227-239. Springer International Publishing, Cham, 2015.

[31] WANG, W.; LIU, Z. ; HU, B.. Phase order in chaotic maps and in coupled map lattices. Physical Review Letters, 84(12):2610-2613, mar 2000.

[32] ZHUANG, G.; WANG, J.; SHI, Y. ; WANG, W.. Phase synchronization and its cluster feature in two-dimensional coupled map lattices. Physical Review E, 66(4), oct 2002. 